Um estudo sobre o papel de medidas de similaridade em visualização de coleções de documentos

Frizzi Alejandra San Roman Salazar 
SERVIÇO DE PÓS-GRADUAÇÃO DO ICMC-USP

Data de Depósito: 23/11/2012

Assinatura:

\title{
Um estudo sobre o papel de medidas de similaridade em visualização de coleções de documentos
}

\author{
Frizzi Alejandra San Roman Salazar
}

Orientadora: Profa. Dra. Maria Cristina Ferreira de Oliveira

Dissertação apresentada ao Instituto de Ciências Matemáticas e de Computação - ICMC-USP, como parte dos requisitos para obtenção do título de Mestre em Ciências Ciências de Computação e Matemática Computacional. VERSÃO REVISADA

USP - São Carlos

Novembro de 2012 
Ficha catalográfica elaborada pela Biblioteca Prof. Achille Bassi e Seção Técnica de Informática, ICMC/USP, com os dados fornecidos pelo(a) autor(a)

San Roman Salazar, Frizzi Alejandra

Um Estudo sobre o Papel de Medidas de

Similaridade em Visualização de Coleções de Documentos

/ Frizzi Alejandra San Roman Salazar; orientadora

Maria Cristina Ferreira de Oliveira. -- São Carlos, 2012 .

$101 \mathrm{p}$.

Dissertação (Mestrado - Programa de Pós-Graduação em Ciências de Computação e Matemática Computacional) -Instituto de Ciências Matemáticas e de Computação, Universidade de São Paulo, 2012.

1. Análise Visual de Textos. 2. Mineração Visual de Textos. 3. Modelo do Espaço Vetorial. 4.

Visualização de Dados de Alta Dimensionalidade. 5. Projeções Multidimensionais. I. Ferreira de Oliveira, Maria-Gristina, orient. II. Titulo. 

Papapo... lo logramos!! 
a Dios, a la Virgen María y al niño Jesús, por todas las bendiciones a mí concedidas; a mi abuelito Victor, por toda la inspiración y buen ejemplo. Gracias papapo porque desde el cielo acompañas mis días, me fortaleces y cuidas; a mi mamá June y a mi abuelita Emma por sus oraciones, su amor y la confianza en mí depositada. A mis hermanos Christian, Valery y Ayline por todo su cariño. Un +1 a Aylincita por dar soporte técnico en las videoconferencias. A mis primitas Valentina y Jimena por sus cartitas que me alegraban los días. A mis papás, mis tíos(as) y primos(as) por sus buenos deseos; a Erick por todo el apoyo, la confianza y cariño durante estos años... las cosas hubieran sido diferentes sin ti;

à minha orientadora, Profa. Dra. Maria Cristina Ferreira de Oliveira, por me aceitar como sua aluna acreditando na minha capacidade, pela excelente orientação fornecida, apoio e parceria na realização deste projeto; ao Instituto de Ciências Matemáticas e de Computação ICMC-USP, pela oportunidade de realização do curso de mestrado. Aos funcionários e professores sempre dispostos na prestação de seus serviços, especialmente à Profa. Dra. Rosane Minghim pela colaboração, os conselhos e amizade; aos meus colegas de laboratório VICG pela ajuda indispensável nesses anos de mestrado; ao Roberto Dantas de Pinho por disponibilizar o software para o iVSM; aos amigos que fiz em São Carlos e a todos que, direta o indiretamente, contribuíram para que este trabalho fosse possível; 
ao Conselho Nacional de Desenvolvimento Científico e Tecnológico (CNPq) e à Fundação de Amparo à Pesquisa do Estado de São Paulo (FAPESP) pela concessão da bolsa de mestrado e pelo apoio financeiro para a realização desta pesquisa, processos 133874/2010-9 e 2010/03100-8. 


\section{SAN ROMAN, Frizzi. Um Estudo sobre o Papel de Medidas de} Similaridade em Visualização de Coleções de Documentos. 2012. Dissertação (Mestrado) - Instituto de Ciências Matemáticas e de Computação, Universidade de São Paulo, São Carlos, 2012.

Técnicas de visualização de informação, tais como as que utilizam posicionamento de pontos baseado na similaridade do conteúdo, são utilizadas para criar representações visuais de dados que evidenciem certos padrões. Essas técnicas são sensíveis à qualidade dos dados, a qual, por sua vez, depende de uma etapa de pré-processamento muito influente. Esta etapa envolve a limpeza do texto e, em alguns casos, a detecção de termos e seus pesos, bem como a definição de uma função de (dis)similaridade. Poucos são os estudos realizados sobre como esses cálculos de (dis)similaridade afetam a qualidade das representações visuais geradas para dados textuais. Este trabalho apresenta um estudo sobre o papel das diferentes medidas de (dis)similaridade entre pares de textos na geração de mapas visuais. Nos concentramos principalmente em dois tipos de funções de distância, aquelas computadas a partir da representação vetorial do texto (Vector Space Model (VSM)) e em medidas de comparação direta de strings textuais. Comparamos o efeito na geração de mapas visuais com técnicas de posicionamento de pontos, utilizando as duas abordagens. Para isso, foram utilizadas medidas objetivas para comparar 
a qualidade visual dos mapas, tais como Neighborhood Hit (NH) e Coeficiente de Silhueta (CS). Descobrimos que ambas as abordagens têm pontos a favor, mas de forma geral, o VSM apresentou melhores resultados quanto à discriminação de classes. Porém, a VSM convencional não é incremental, ou seja, novas adições à coleção forçam o recálculo do espaço de dados e das dissimilaridades anteriormente computadas. Nesse sentido, um novo modelo incremental baseado no VSM (Incremental Vector Space Model (iVSM)) foi considerado em nossos estudos comparativos. O iVSM apresentou os melhores resultados quantitativos e qualitativos em diversas configurações testadas. Os resultados da avaliação são apresentados e recomendações sobre a aplicação de diferentes medidas de similaridade de texto em tarefas de análise visual, são oferecidas.

Palavras-chave: Análise Visual de Textos, Mineração Visual de Textos, Modelo do Espaço Vetorial, Visualização de Dados de Alta Dimensionalidade, Projeções Multidimensionais. 
SAN ROMAN, Frizzi. A Study on the Role of Similarity Measures in Visual Text Analytics. 2012. Dissertation (Master) Institute of Mathematics and Computer Science, University of São Paulo, São Carlos, 2012.

Information visualization techniques, such as similarity based point placement, are used for generating of visual data representation that evidence some patterns. These techniques are sensitive to data quality, which depends of a very influential preprocessing step. This step involves cleaning the text and in some cases, detecting terms and their weights, as well as definiting a (dis)similarity function. There are few studies on how these (dis)similarity calculations affect the quality of visual representations for textual data. This work presents a study on the role of the various (dis)similarity measures in generating visual maps. We focus primarily on two types of distance functions, those based on vector representations of the text (Vector Space Model (VSM)) and measures obtained from direct comparison of text strings, comparing the effect on the visual maps obtained with point placement techniques with the two approaches. For this, objective measures were employed to compare the visual quality of the generated maps, such as the Neighborhood Hit and Silhouette Coefficient. We found that both approaches have strengths, but in general, the 
VSM showed better results as far as class discrimination is concerned. However, the conventional VSM is not incremental, i.e., new additions to the collection force the recalculation of the data space and dissimilarities previously computed. Thus, a new model based on incremental VSM (Incremental Vector Space Model (iVSM)) has been also considered in our comparative studies. iVSM showed the best quantitative and qualitative results in several of the configurations considered. The evaluation results are presented and recommendations on the application of different similarity measures for text analysis tasks visually are provided.

Keywords: Visual Text Analytics, Visual Text Mining, Vector Space Model, High-dimensional Data Visualization, Multidimensional Projections. 
1 Introdução 3

2 Visualização de Coleções de Documentos 9

2.1 Considerações Iniciais . . . . . . . . . . . . . . . . . . . . . . 9

2.2 TextPool . . . . . . . . . . . . . . . . . . . . . . 10

2.3 Metáfora do Fluxo de Rio . . . . . . . . . . . . . . . . . . 12

2.4 Tag Clouds ............................. 14

2.5 Incremental Board . . . . . . . . . . . . . . . . . . . . . . 19

2.6 Considerações Finais . . . . . . . . . . . . . . . . . . 21

3 Cálculo de Dissimilaridade entre Documentos 25

3.1 Considerações Iniciais . . . . . . . . . . . . . . . . . . . . 25

3.2 Métodos de Representação de Textos . . . . . . . . . . . . . . . . 26

3.2 .1 Modelo Vetorial . . . . . . . . . . . . . . . . . . . . . 26

3.2 .2 Modelo Vetorial Incremental . . . . . . . . . . . . . . . . . . . . 29

3.3 Medidas de Dissimilaridade entre Documentos . . . . . . . . . . . . . . . 30

3.4 Considerações Finais . . . . . . . . . . . . . . . . . . . . . . . . . 38 
4 Técnicas de Posicionamento de Pontos 41

4.1 Considerações Iniciais . . . . . . . . . . . . . . . . . . . . . . 41

4.2 Técnicas de Projeção Multidimensional . . . . . . . . . . . . . . . . . . 41

4.2.1 Interactive Document Map . . . . . . . . . . . . . . . . . . . . . 43

4.2.2 Projection by Clustering . . . . . . . . . . . . . . . . . . . 43

4.2.3 Least Square Projection . . . . . . . . . . . . . . . . . . . . . . 44

4.3 Árvores Filogenéticas . . . . . . . . . . . . . . . . . . . . . . 45

4.3.1 Neighbor Joining . . . . . . . . . . . . . . . . . . . . 46

4.4 Medidas de Avaliação da Qualidade de Mapas Visuais . . . . . . . . . . . . 48

4.4 .1 Coeficiente de Silhueta . . . . . . . . . . . . . . . . . . . . . . . 48

4.4.2 Neighborhood Hit . . . . . . . . . . . . . . . . . . . . . . . . . . . . 49

4.5 Considerações Finais . . . . . . . . . . . . . . . . . . . . . . 51

5 Descrição do Estudo Experimental 53

5.1 Considerações Iniciais . . . . . . . . . . . . . . . . . . . . . . . 53

5.2 Módulos Desenvolvidos no VisPipeline . . . . . . . . . . . . . . 55

5.3 Coleções de Textos . . . . . . . . . . . . . . . . . . . . 57

5.4 Medidas para o Cálculo da Similaridade entre Strings . . . . . . . . . . . . 60

5.5 Configuração dos Experimentos . . . . . . . . . . . . . . . . . . . . . 62

5.5.1 Configuração do Modelo de Linguagem . . . . . . . . . . . . . . . . 62

5.5.2 Configuração das Medidas de Similaridade de Strings . . . . . . . . 63

5.6 Considerações Finais . . . . . . . . . . . . . . . . . . . . . . . 65

$\begin{array}{llr}6 & \text { Resultados } & 69\end{array}$

6.1 Considerações Iniciais . . . . . . . . . . . . . . . . . . . . . . . . . . . . . . 69

6.2 Experimento 1: Comparando Medidas sobre Strings . . . . . . . . . . . . . 70

6.3 Experimento 2: Comparando o VSM e iVSM . . . . . . . . . . . . . . . . 74

6.4 Experimento 3: Comparando Medidas sobre Strings e sobre Modelos Vetoriais 82

6.5 Considerações Finais . . . . . . . . . . . . . . . . . . . . . . . 84

$\begin{array}{lll}7 & \text { Conclusões } & 91\end{array}$

7.1 Limitações e Trabalhos Futuros . . . . . . . . . . . . . . . . . . . . 92

$\begin{array}{ll}\text { Referências Bibliográficas } & 93\end{array}$ 


\section{Lista de Figuras}

1.1 Projeção multidimensional de uma coleção de artigos científicos gerada com a plataforma Projection Explorer (PEx) (Paulovich et al., 2007) utilizando a técnica de projeção ProjClus (Paulovich e Minghim, 2006) e a distância de cosseno. As cores representam a classe do documento. Documentos com conteúdo similar são posicionados próximos no plano de visualização. . . .

2.1 Relacionamento entre termos salientes no TextPool. Os vértices representam os termos mais frequentes na coleção e as arestas indicam a coocorrência dos termos nos documentos. Fonte (Albrecht-Buehler et al., 2005). . . . . . . . . . . . . . . . . . . 10

2.2 ThemeRiver adota a metáfora de um 'rio' para representar temas em uma coleção de documentos. A figura representa a visualização gerada a partir de uma coleção de documentos referentes à crise cubana-americana, contendo discursos do Fidel Castro, entrevistas e artigos desde finais de 1959 a meados de 1961. Extraído de (Havre et al., 2002) . . . . . . . . . . . . . . 13

2.3 Visualização de um conjunto de documentos (e-mails) utilizando TIARA. No layout gerado, cada camada do 'rio' representa um tópico o qual é descrito por um conjunto de palavras chaves. Para cada tópico, a largura da camada está relacionada com o número de documentos do tópico em um determinado momento. Extraído de (Wei et al., 2010). . . . . . . . . . 14

2.4 Visualização Tag Cloud para uma coleção de notícias, utilizando a ferramenta PEx (Paulovich et al., 2007). . . . . . . . . . . . . . . . . . . . 15 
2.5 Representação gerada pela técnica de Cui et al. (2010) para uma coleção de documentos dinâmica. As Figuras 2.5a até 2.5e representam cinco nuvens de palavras construídas referentes a cinco diferentes intervalos de tempo. A Figura 2.5f apresenta um gráfico de frequência de uma palavra da coleção, para análise da sua evolução entre Maio de 1995 e Junho de 2007. Fonte Cui et al. (2010). . . . . . . . . . . . . . . . . . . . . . . 16

2.6 Visualização gerada com a ProjCloud: nuvens de palavras são geradas dos grupos identificados na projeção multidimensional. Fonte Paulovich et al. (2012)

2.7 Três momentos na construção incremental do mapa de um corpus dinâmico de documentos utilizando a técnica Incremental Board (incBoard). As cores representam as classes dos documentos. Fonte (Pinho, 2009) . . . . . . . .

3.1 Cortes de Luhn aplicados a uma curva de Zipf para uma coleção de artigos científicos, gerada com a plataforma Projection Explorer (PEx) (Paulovich et al., 2007). O eixo cartesiano $r$ representa os termos da coleção e o eixo cartesiano $f$ representa a frequência desses termos. . . . . . . . . . . 28

4.1 Mapa do conjunto de documentos CBR-ILP-IR gerado com a técnica Interactive Document Map (IDMAP) . . . . . . . . . . . . . . . . . 44

4.2 Mapa gerado para o conjunto CBR-ILP-IR usando Projection by Clustering (ProjClus). . . . . . . . . . . . . . . . . . . . . 44

4.3 Mapa de documentos do CBR-ILP-IR gerado com a técnica Least Square Projection (LSP) . . . . . . . . . . . . . . . . . . . 45

4.4 Árvore filogenética gerada com a ferramenta MATLAB . . . . . . . . . . 46

4.5 Mapa de documentos do conjunto CBR-ILP-IR gerado com a técnica Neighbor Joining $(\mathrm{NJ}) \ldots \ldots \ldots \ldots$. . . . . . . . . . . . . . . . . . . 4 48

4.6 Quatro diferentes projeções com seus respectivos coeficientes de silhueta para o conjunto de artigos científicos. . . . . . . . . . . . 50

4.7 Curvas de precisão de diferentes técnicas para a geração de mapas visuais usando matrizes de distância calculadas com NCD. Extraído de (Cuadros,

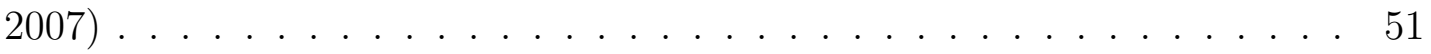

5.1 Pipeline de visualização f . . . . . . . . . . . . . . . . . 54

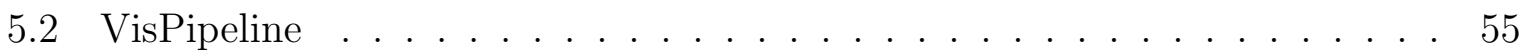


5.3 VisPipeline: Pipeline criado para computar uma matriz de dissimilaridade entre documentos, utilizando o módulo String Metrics. . . . . . . . . . . . 56

5.4 VisPipeline: Pipeline para computar do modelo iVSM dos documentos de uma coleção. . . . . . . . . . . . . . . . . . . . . . . 57 57

5.5 As Figuras 5.5(a) e 5.5(a) apresentam um exemplo dos feeds RSS das notícias topo dos fornecedores de notícias Reuters e CNN.com, respectivamente. 58

5.6 Tempos de processamento de diferentes medidas de comparação de strings, no cálculo da dissimilaridade entre duas strings de igual tamanho. A Figura 5.6(a) apresenta os gráficos gerados para as quinze métricas utilizadas. A Figura 5.6(b) apresenta o gráfico anterior, excluindo a medida MongeElkan. 61

5.7 Gráficos de precisão para o conjunto CBR-ILP-IR . . . . . . . . . . . . . . 64

5.8 Gráficos de precisão para o conjunto News2011 . . . . . . . . . . . . . . . . 65

5.9 Tempos de processamento em segundos, para o cálculo da matriz de dissimilaridade utilizando diferentes métricas de comparação de strings com e sem remoção de stopwords. . . . . . . . . . . . . . . . . . . . .

6.1 Resultados de precisão das projeções do conjunto de artigos científicos CBR-ILP-IR . . . . . . . . . . . . . . . . . . . . 72

6.2 Resultados de precisão das projeções do conjunto de notícias News2011 . 73

6.3 Ranking dos cinco termos mais frequentes no modelo vetorial incremental, para o conjunto CBR-ILP-IR, em diferentes instantes de tempo. . . . . . .

6.4 Ranking das cinco primeiras palavras com maior DF no modelo vetorial incremental, para conjunto de textos News2011, para diferentes instantes de tempo. . . . . . . . . . . . . . . . . . .

6.5 Resultados de precisão das projeções LSP geradas para o conjunto do artigos CBR-ILP-IR. . . . . . . . . . . . . . . . . .

6.6 Projeções multidimensionais para o conjunto de dados CBR-ILP-IR. Ambas as projeções apresentam misturas entre as classes existentes no conjunto de dados, por exemplo, os elementos indicados no interior dos circulo azul claro e laranja. Elementos das três classes ficaram mais próximos entre si no layout gerado com o modelo iVSM, o que permite identificar claramente os três grupos, mas isso também provoca maior sobreposição de pontos que na projeção gerada com o modelo vetorial, o que poderia tornar difícil uma análise local na classe. . . . . . . . . . . . . . . . . . . . . . . 77 
6.7 Resultados de precisão das árvores NJ geradas para o conjunto do artigos CBR-ILP-IR, utilizando a medida do cosseno sobre os modelos VSM e iVSM. 78

6.8 As árvores NJ geradas para o conjunto de textos CBR-ILP-IR. As duas árvores refletem as três classes do conjunto de dados, mas com alguma mistura entre as classes. O layout gerado com o modelo incremental apresentou menos mistura entre elementos de diferentes classes, como se observa nos setores indicados pelas circunferências nas cores verde, laranja e azul claro . . . . . . . . . . . . . . . . . . . . . . 79

6.9 Resultados de precisão das projeções LSP geradas para o conjunto News2011. 79

6.10 Projeções multidimensionais LSP para o conjunto de textos News2011. Embora alguma mistura entre grupos seja inevitável, devido ao número de classes na coleção (23 classes), algumas diferenças são identificáveis. Por exemplo, elementos da classe amarela (circunferência preta) ficaram mais bem agrupados no layout gerado com o VSM do que no layout gerado com o iVSM. O mesmo acontece com os elementos das circunferências rosa, roxa e cinza. . . . . . . . . . . . . . . . . . . 80

6.11 Resultados de precisão das árvore NJ geradas para o conjunto News2011. 81

6.12 Árvores NJ geradas para o conjunto de textos News2011. Realizar um análise visual torna-se difícil devido à quantidade de elementos, classes e cores presentes no layout. Na Figura 6.13 uma exploração dessas árvores é realizado, utilizando o módulo Identity Coordination da ferramenta Vispipeline. . . . . . . . . . . . . . . . . . . . . . . . . . . . . 81

6.13 Exploração das árvores NJ do conjunto News2011 utilizando o módulo Identity Coordination. Nas Figuras 6.13(a) e 6.13(b) elementos da cor ciano foram selecionados, observa-se que enquanto o VSM agrupou todos os elementos em um só ramo, o iVSM separou a classe em dois diferentes ramos. Nas Figuras 6.13(c) e 6.13(d) elementos da classe verde limão foram selecionados. Novamente o layout gerado pelo modelo vetorial incremental dividiu a classe em dois ramos distintos. Por último, nas Figuras 6.13(e) e 6.13(f) foram selecionados elementos da classe verde claro. Para esta classe, o layout gerado com o iVSM agrupou melhor os elementos em comparação ao gerado com o VSM, no qual misturou-se com elementos das classes azul, laranja e verde escuro. . . . . . . . . . . . . . . . . . . 86

6.14 Resultados de precisão das projeções geradas para o conjunto CBR-ILP-IR 87 6.15 Resultados de precisão das projeções geradas para o conjunto News2011 . . 88 
6.16 Resultados de precisão das projeções geradas para o conjunto NewsReuters 



\section{Lista de Tabelas}

3.1 Representação do modelo de espaço vetorial de uma coleção hipotética de documentos. Cada linha representa um documento e cada coluna representa um termo no documento. As entradas na tabela indicam a frequência de um termo em um determinado documento. . . . . . . . . . . . . . . 26

3.2 Representação do modelo de linguagem de uma coleção de documentos hipotética. Cada linha representa um termo no modelo vetorial, a coluna $T F$ representa a frequência do termo na coleção e, a coluna $D F$ a quantidade de documentos da coleção nos quais o termo aparece. . . . . . . . . . . . . 29

4.1 Matriz de distâncias. . . . . . . . . . . . . . . . . . . 42

5.1 Descrição do conjunto de dados News2011. . . . . . . . . . . . . . . . . . 59

5.2 Configuração utilizada na geração do modelo vetorial para o conjunto de dados all . . . . . . . . . . . . . . . . . . 62

5.3 Configuração utilizada na geração do modelo vetorial para o conjunto de dados AP_BBC_CNN_Reuters . . . . . . . . . . . . . . . . . 63

5.4 Pré-processamento aplicado (remoção de stopwords) aos textos previamente ao cómputo das métricas de comparação de strings. . . . . . . . . . . . . . 66

6.1 Ranking das curvas NH dos layouts obtidos com métricas baseadas em strings e a distância do cosseno computada sobre os modelos VSM e iVSM, para os três conjuntos de textos. . . . . . . . . . . . . . . . . . . 8 8 

CS Coeficiente de Silhueta

CSP Cutting Stock Problem

IDMAP Interactive Document Map

incBoard Incremental Board

iVSM Incremental Vector Space Model

LSP Least Square Projection

MDS Multidimensional Scaling

NCBI National Center for Biotechnology Information

NH Neighborhood Hit

NJ Neighbor Joining

NMI Informação Mútua Normalizada

NP Neighborhood Preservation

ProjClus Projection by Clustering

VICG Visualization, Imaging and Computer Graphics

VSM Vector Space Model 

A vasta disponibilidade de dados introduz o desafio de criar estratégias e ferramentas que apoiem os seres humanos em tarefas de análise e descoberta de informação nesses dados. Dados 'do mundo real', oriundos de bases de dados, de sensores e dispositivos diversos de medição, geralmente consistem de uma grande quantidade de elementos, cada qual associado a múltiplos atributos, cujo comportamento precisa ser analisado. Adicionalmente, conjuntos de documentos de texto, imagens e vídeos tornam-se amplamente disponíveis provenientes da Web e dos dispositivos ubíquos de captura e armazenamento, que transformam qualquer pessoa comum em uma fonte geradora de dados.

Entretanto, ao contrário da capacidade das máquinas, a nossa capacidade cognitiva permanece estável. O volume, a dimensionalidade e a heterogeneidade desafiam os algoritmos de análise e a capacidade humana de extrair informação relevante dos dados. Essa dificuldade permeia diferentes disciplinas e domínios, tanto no cenário da pesquisa acadêmica como nos setores de serviços, industrial e agro-pastoril. De fato, a gestão da informação em grandes volumes de dados multimídia distribuídos foi identificada como um dos Grandes Desafios de pesquisa em Computação no Brasil ${ }^{1}$. A análise dos dados é, certamente, um dos aspectos deste problema, como apontado no II Seminário da Sociedade Brasileira de Computação realizado sobre o tema ${ }^{2}$.

\footnotetext{
${ }^{1}$ http://www.sbc.org.br

${ }^{2}$ http://gd2.ufam.edu.br/
} 
Nesse cenário, é fundamental melhorar o apoio oferecido às pessoas em processos de exploração de dados. É essa a proposta da Visualização Computacional, disciplina que surgiu a partir da disseminação dos recursos computacionais capazes de gerar gráficos interativos (Card et al., 1999; Chen, 2006; Fayyad et al., 2002; de Oliveira e Levkowitz, 2003). Podem-se identificar duas vertentes nessa área de pesquisa: Técnicas de Visualização Científica que englobam, em geral, a criação de modelos gráficos de dados oriundos do mundo físico, obtidos do corpo humano, do planeta terra, de modelos moleculares, entre outros. Tais dados embutem informação inerentemente geométrica, a qual é determinante na geração das representações visuais. Técnicas de Visualização de Informação que mapeiam, em geral, dados de natureza não física, como por exemplo, dados financeiros, coleções de documentos e outras concepções abstratas.

A Visualização de Informação tem seu potencial maximizado quando é acoplada a estratégias analíticas, oriundas da Estatística e Mineração, como reconhecido em recente iniciativa norte-americana de fomentar a pesquisa em Visual Analytics (Thomas e Cook, 2006). Da mesma forma, hoje admite-se que, para serem úteis em cenários complexos, mesmo algoritmos 'automáticos' de mineração ou reconhecimento de padrões devem (ou precisam) admitir a interferência do usuário para viabilizar a busca por uma solução. A integração de algoritmos de mineração e visualização permite agregar a capacidade computacional ao conhecimento e capacidade humanas de perceber padrões, de modo a conceber ambientes adequados para usuários extraírem informações dos dados de maneira mais efetiva (de Oliveira e Levkowitz, 2003).

Assim, técnicas de mineração de dados e de visualização de informação têm sido aplicadas a dados abstratos, no que denomina-se mineração visual de dados. Um exemplo da utilização dessas técnicas é a construção de representações visuais que mapeiam coleções de documentos textuais em espaços bi ou tridimensionais.

Uma opção para visualizar conjuntos de documentos é posicionar marcadores gráficos que os representam em um espaço bidimensional seguindo relações de similaridade ou dissimilaridade de conteúdo. Assim, visualizações baseadas em posicionamento de pontos ou projeções multidimensionais mapeiam um conjunto de elementos gráficos no plano. Elementos posicionados próximos nesse plano representam documentos que, de acordo com a medida de (dis)similaridade escolhida, tem conteúdo semelhante. Pelo contrário, pontos projetados distantes, indicam documentos com conteúdo pouco relacionado. A Figura 1.1 apresenta um exemplo de projeção multidimensional de uma coleção de 574 artigos científicos, distribuídos em três áreas de pesquisa distintas. A cor do círculo indica 
a classe à qual o documento pertence: Case-Based Reasoning na cor vermelha, Inductive Logic Programming em verde e Information Retrieval em azul.

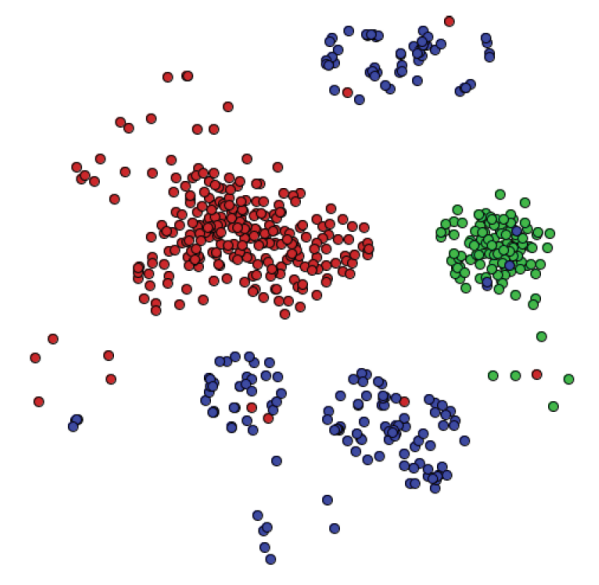

Figura 1.1: Projeção multidimensional de uma coleção de artigos científicos gerada com a plataforma Projection Explorer (PEx) (Paulovich et al., 2007) utilizando a técnica de projeção ProjClus (Paulovich e Minghim, 2006) e a distância de cosseno. As cores representam a classe do documento. Documentos com conteúdo similar são posicionados próximos no plano de visualização.

A concepção de visualização para documentos textuais requer um passo de pré-processamento em que a representação subjacente e medições de similaridade desempenham um papel importante. Devido à sua natureza, a manipulação de documentos requer uma representação que descreva a informação contida neles de forma estruturada, com a mínima perda de informação possível. Geralmente, o modelo VSM (Vector Space Model) é utilizado com esse objetivo (Salton et al., 1975). Essa representação considera a frequência de palavras ou termos considerados relevantes e também é conhecida como "bag-ofwords". Sobre esta representação vetorial, a distância do cosseno é usualmente empregada como uma aproximação de dissimilaridade entre um par de textos. Apesar de ser uma abordagem amplamente adotada, não existem estudos sistemáticos sobre como este préprocessamento afeta o resultado das técnicas de visualização de texto.

Alternativamente, diversas medidas de similaridade entre textos baseadas na comparação de direta de strings, que dependem apenas de um par de documentos, estão disponíveis na literatura. Poucos são os estudos realizados sobre como essas alternativas afetam a análise de texto, de maneira visual ou não.

Neste trabalho realizamos um estudo de como o pré-processamento de texto, particularmente a escolha de uma função de (dis)similaridade, afeta a saída produzida por visualizações de documentos baseadas em similaridade de conteúdo. Tomamos como base 
visualizações que apresentam documentos como pontos em um plano com base na sua semelhança, verificando como as diferentes escolhas de medidas de (dis)similaridade afetam a qualidade de tais visualizações, em termos de agrupamento e separação de textos com conteúdo altamente relacionado.

A abordagem padrão para a representação vetorial de textos apresenta muitas limitações para efeitos de visualização. Por exemplo, o VSM não consegue captar a semântica implícita nas relações entre palavras e termos. Além disso, a construção de um VSM requer operações como a identificação de palavras consideradas não relevantes, geralmente através de uma lista de stopwords, e possivelmente, a aplicação de algoritmos para a lematização de palavras. Esse pré-processamento exige a intervenção do usuário e configurações de parâmetros que podem afetar o resultado consideravelmente, tornando mais difícil o processo de obter visualizações automaticamente.

Ademais, o cálculo do VSM de uma coleção requer que todos os documentos estejam disponíveis a priori, o que representa uma grande limitação, pois muitos fenômenos importantes geram dados continuamente e com diferentes tipos de atualização, tais como a adição ou remoção elementos. Em uma visualização cuja entrada é o VSM, a adição de um novo documento forçará o recálculo das similaridades previamente computadas mudando assim a localização dos documentos no espaço visual. Uma alternativa é empregar medidas de distâncias entre strings, que não requerem o VSM. Muitas dessas medidas foram definidas para diferentes fins e aplicações (Cohen et al., 2003; Gravano et al., 2001; Huang e Madey, 2004; Kempken et al., 2006). Assim, a adição de um novo elemento à coleção requer unicamente comparações do novo documento com os já existentes. A questão permanece de como tais medidas, em comparação com a distância do cosseno calculada sobre o VSM, afetam a qualidade das representações visuais.

Esta dissertação investiga estas questões, buscando respostas as seguintes perguntas:

1. Medidas de distância entre strings são adequadas para a visualização de textos com base na similaridade? Quais medidas poderiam ser consideradas neste contexto, e como diferentes medidas de distância afetam as visualizações?

2. É possível adotar um VSM modificado para representar uma coleção dinâmica de documentos textuais (modelo proposto por Pinho (2009)), construindo um modelo vetorial que pode ser atualizado conforme os documentos são adicionados à coleção?

3. Como esse modelo modificado afeta o resultado das visualizações baseadas na semelhança, em comparação com visualizações obtidas com o VSM convencional e com medidas de distância entre strings?

Assim, esta dissertação está estruturada da seguinte maneira: 
- No Capítulo 2 é apresentada uma revisão bibliográfica sobre ferramentas na área de Visualização de Informação que visam gerar representações visuais de coleções de documentos textuais.

- O Capítulo 3, por sua vez, traz uma revisão bibliográfica sobre representações de documentos e métricas de cálculo de dissimilaridade entre textos.

- No Capítulo 4 são apresentadas as técnicas de projeção multidimensional e árvores filogenéticas mais relevantes para esse trabalho. Nesse capítulo também são apresentadas medidas para avaliar a qualidade das representações visuais geradas.

- No Capítulo 5 são descritos os elementos e as configurações utilizadas para o desenvolvimento do estudo experimental realizado.

- No Capítulo 6 são apresentados os resultados do estudo comparativo realizado sobre diferentes conjuntos de textos.

- Finalmente, no Capítulo 7 apresenta-se as conclusões desse projeto de mestrado, caracterizando as suas contribuições e limitações. 


\section{Visualização de Coleções de Documentos}

\subsection{Considerações Iniciais}

O avanço da tecnologia e dos equipamentos de aquisição de informação tem disponibilizado um volume enorme de informações de diversas origens e formatos. Grande parte das informações disponíveis atualmente é representada na forma de documentos. Revistas, artigos, jornais, notícias e mensagens eletrônicas são exemplos de meios que utilizam textos para transmitir informações a seus leitores. Além disso, muitos fenômenos importantes geram dados continuamente, ou seja, geram streams de dados. Dessa maneira, muitas coleções de documentos disponíveis são dinâmicas, ou seja, evoluem a medida que novos elementos são gradualmente acrescentados à coleção.

Este excesso de informação pode tornar difícil buscar e explorar informações. Nesse cenário, é importante dispor de representações visuais que aceitem coleções de documentos dinâmicas como entrada e consigam detectar e evidenciar mudanças de comportamento, ao longo do tempo. Neste capítulo apresenta-se algumas das ferramentas e técnicas de visualização utilizadas recentemente para este fim. 


\subsection{TextPool}

TextPool (Albrecht-Buehler et al., 2005) é um dos primeiros sistemas de visualização capazes de detectar e refletir a evolução temporal de uma coleção de documentos. O sistema utiliza um buffer temporal para visualizar streams de texto em tempo real, tais como notícias de ultima hora oferecidas por diferentes serviços na internet. Esse buffer temporal permite ao usuário manter-se atualizado com as últimas atualizações apresentadas na visualização, sem perder as anteriores, assim pode-se ter controle sobre a idade da visualização. Nesse contexto, o usuário poderia observar quais foram os tópicos discutidos nas últimas duas horas, nos últimos dois dias ou nas últimas duas semanas.

Para o caso das notícias, são incorporados resumos curtos que descrevem de forma compacta o documento completo. A técnica utiliza esses resumos e aplica uma remoção de palavras irrelevantes (stop words) para computar de maneira simples um vetor conteúdo que contempla as palavras importantes que representam os documentos. Uma palavra é definida como importante quando a frequência com a que aparece no stream é alta.

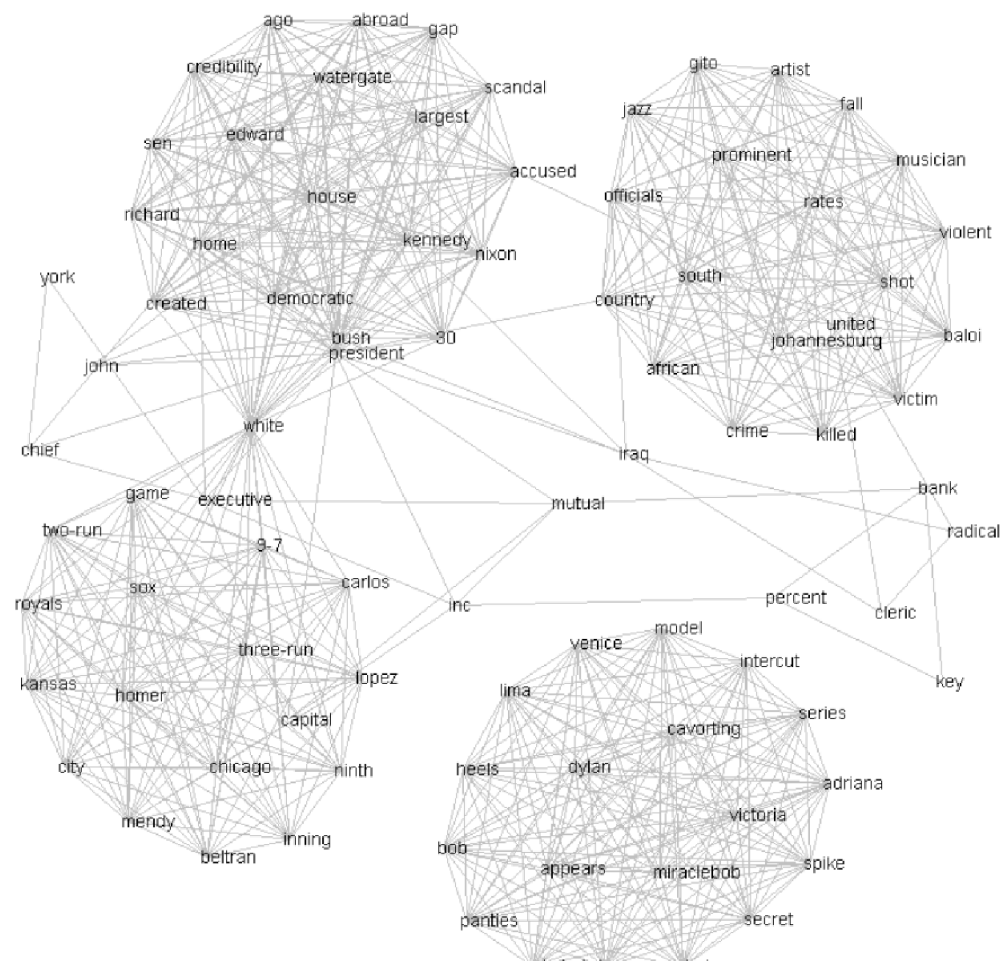

Figura 2.1: Relacionamento entre termos salientes no TextPool. Os vértices representam os termos mais frequentes na coleção e as arestas indicam a co-ocorrência dos termos nos documentos. Fonte (Albrecht-Buehler et al., 2005). 
Esta técnica gera um grafo, sendo que cada vértice representa os termos mais frequentes a partir dos vetores conteúdo do stream e as arestas indicam a co-ocorrência das palavras. O comprimento das arestas é inversamente proporcional ao número de vezes que as palavras co-ocorrem. Assim, palavras muito relacionadas e que co-ocorrem com frequência são localizados próximos no grafo. A figura 2.1 mostra este relacionamento. O grafo representa somente os termos recentes, usando uma janela temporal controlada pelo usuário. Dessa forma, um documento pode expirar, alterando os valores de co-ocorrência entre palavras e aumentando a distância entre eles. Um termo também pode desaparecer, a transição ocorre lentamente (fades out) e os termos restantes movem-se suavemente para ocupar o espaço deixado vago na visualização. Quando um novo termo é agregado, ele é inserido primeiro na borda do grafo, e vai sendo lentamente reposicionado de modo a refletir os seus relacionamentos com os demais termos.

A técnica é animada de acordo com a mudança temporal dos documentos utilizando técnicas de posicionamento baseado em forças (force-directed layout). O grafo é modelado como um sistema físico massa-mola, no qual os vértices são tratados como partículas com massa e as arestas se comportam como molas. Cada mola exerce uma força proporcional à diferença entre o tamanho atual da aresta e o seu tamanho ideal indicado pela coocorrência, fazendo com que cada massa mova-se na direção da soma das forças exercidas sobre ela. O grafo é atualizado iterativamente na tentativa de estabilizar o sistema. As iterações do force-directed layout proporcionam a sensação de animação provocada pela mudança dos termos ao longo do tempo.

Os autores apresentam uma técnica com capacidade de controlar stream de textos com altas taxas de transmissão de dados, i.e., mais de 50,000 documentos por dia. Além disso, o sistema é capaz de prevenir o fenômeno conhecido como change blindness ${ }^{1}$ em suas animações. Assim, os usuários podem acompanhar as múltiplas mudanças dos dados na visualização.

A desvantagem do método consiste no fato de que os vértices do grafo são rotulados pelos termos importantes, assim quando o número de vértices é grande, a sobreposição dos termos pode gerar oclusão visual dificultando a interpretação do gráfico. A visualização apresentada pelo sistema só exibe as palavras importantes no conjunto e não os documentos da coleção. Os autores não apresentam informação sobre o custo computacional.

\footnotetext{
${ }^{1}$ change blindness é o fenômeno que ocorre quando uma pessoa observa uma cena e não detecta as mudanças na cena.
} 


\subsection{Metáfora do Fluxo de Rio}

ThemeRiver Havre et al. (2002) é uma das primeiras técnica de visualização baseadas na metáfora do 'rio', proposta para descrever mudanças temáticas ocorridas em um conjunto de documentos ao longo do tempo. Esse 'rio' é composto por bandas coloridas, que representam temas selecionados e cuja largura indica o número de ocorrências do tema na coleção em um determinado instante de tempo. A linha de tempo acompanha o 'rio' (parte inferior) ao longo da sua existência. A distância horizontal entre dois pontos no 'rio' define um intervalo de tempo (ou momento temporal). Temas podem desaparecer e depois ressurgir com a mesma cor e posição em relação aos outros temas. Existem momentos temporais em que o rio pode secar por completo, isso acontece porque o 'rio' pode representar apenas um subconjunto de temas de uma coleção de documentos, normalmente uma dúzia de temas selecionados. Este subconjunto pode refletir apenas uma pequena parte do conteúdo da coleção completa. A visualização ThemeRiver permite também adicionar marcadores para eventos históricos, o que permite reconhecer padrões que sugerem relações ou tendências relacionados com as mudanças temáticas. A Figura 2.2 expõe uma visualização gerada com o ThemeRiver de um conjunto de documentos referentes à crise cubana-americana ocorrida em 1960.

O ThemeRiver é utilizando como exemplo de visualização de textos para encontrar padrões relevantes, relacionamentos e tendências entre os temas de uma coleção de documentos. Os autores não esclarecem como são selecionados os temas a ser visualizados, o que considera-se uma etapa importante e crítica. Aparentemente é escolha esta baseada no número de ocorrências (ou frequência) de um tema na coleção. A técnica não é incremental, pois toda a coleção deve ser conhecida no momento de gerar o 'rio', impossibilitando a inserção de novos documentos na representação sem que esta seja refeita.

Outro sistema que busca gerar visualizações interativas capazes de detectar e refletir a evolução temporal de uma coleção de documentos utilizando a metáfora do 'rio' é o TIARA (Text Insight via Automated Responsive Analytics) (Wei et al., 2010). Dada uma coleção de documentos, o sistema usa uma técnica de análise de tópicos para resumir os documentos em um conjunto de temas, cada um dos quais é representado por um conjunto de palavras chave, apresentadas na visualização como uma nuvem de palavras. A arquitetura do sistema abrange um módulo de processamento offline e outro online. Como parte do processamento offline, o sistema coleta os documentos de texto de um sistema de arquivos ou uma base de dados, esses documentos são processados por um componente de pré-processamento de textos. O pré-processamento inclui a remoção de 


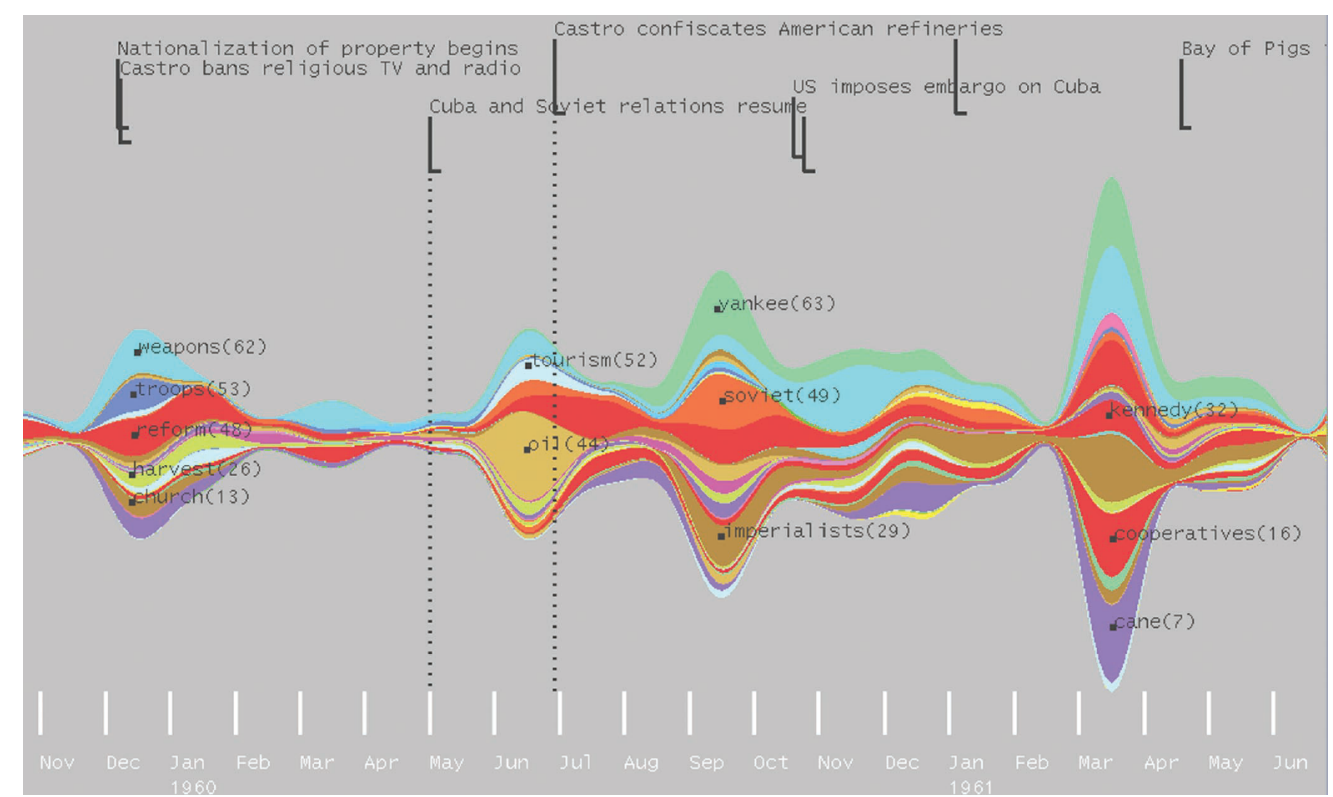

Figura 2.2: ThemeRiver adota a metáfora de um 'rio' para representar temas em uma coleção de documentos. A figura representa a visualização gerada a partir de uma coleção de documentos referentes à crise cubana-americana, contendo discursos do Fidel Castro, entrevistas e artigos desde finais de 1959 a meados de 1961. Extraído de (Havre et al., 2002).

palavras irrelevantes e remoção de assinaturas eletrônicas (no caso de correios eletrônicos). Este componente geralmente precisa de personalização, dependendo da natureza dos documentos. Dos documentos já processados, são extraídos os tópicos chaves e logo indexados a seus documentos utilizando Lucene ${ }^{2}$.

O processamento online é iniciado, geralmente, por causa de uma requisição do usuário, por exemplo, uma consulta por palavras chaves (Figura 2.3a). O sistema analisa a consulta e a converte em uma consulta Lucene compatível, a qual é utilizada para encontrar os documentos correspondentes a partir do índice do texto. Quando os documentos são recuperados, duas visões são apresentadas: uma visão de lista (ver Figura 2.3b) que mostra fragmentos dos documentos que correspondem à consulta do usuário; e uma visão dos tópicos (ver Figura 2.3c) que sumariza visualmente os documentos recuperados como um gráfico de bandas empilhadas.

\footnotetext{
${ }^{2}$ Lucene, é um software de busca e uma API de indexação de documentos, escrito na linguagem de programação Java. É um software de código aberto da Apache Software Foundation. Disponível em http://lucene.apache.org/
} 


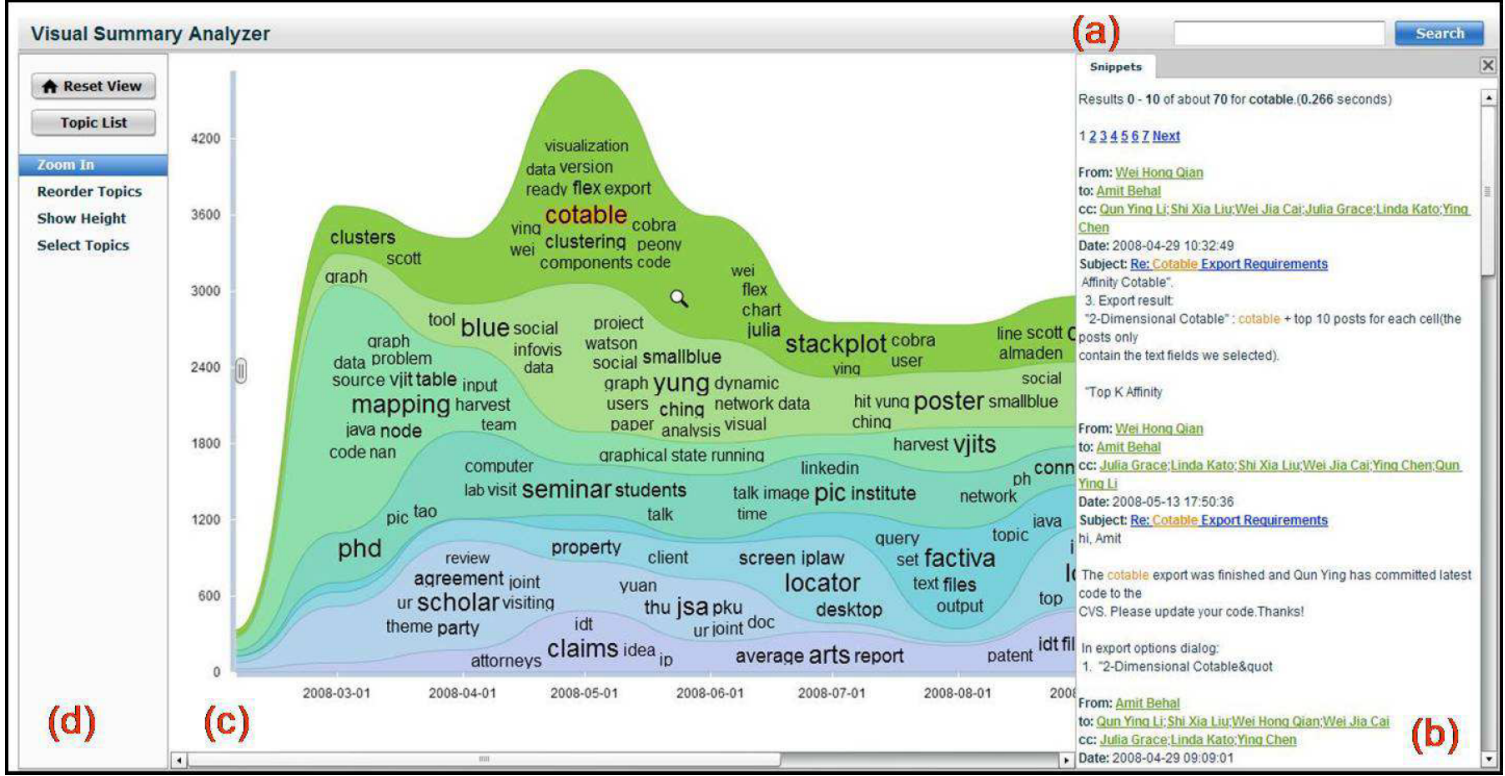

Figura 2.3: Visualização de um conjunto de documentos (e-mails) utilizando TIARA. No layout gerado, cada camada do 'rio' representa um tópico o qual é descrito por um conjunto de palavras chaves. Para cada tópico, a largura da camada está relacionada com o número de documentos do tópico em um determinado momento. Extraído de (Wei et al., 2010).

\subsection{Tag Clouds}

Outra abordagem para gerar uma representação visual de coleções de documentos dinâmicas é utilizando Tag Cloud. Uma Tag Cloud é uma técnica de visualização que visa resumir o conteúdo de documentos textuais com o objetivo de facilitar a interpretação pelo ser humano. A técnica representa as palavras de uma coleção de documentos (ou de um só documento) dentro de regiões retangulares cujo tamanho, assim como o tamanho da fonte da palavra, indica a sua popularidade ou frequência (Hopper e Turton, 2001; Korf, 2004). A Figura 2.4 mostra parte de uma Tag Cloud gerada para uma coleção de 2684 artigos jornalísticos disponibilizados na Internet pelas agências Reuters, BBC, CNN e AP coletados durante dois dias em Abril de 2006. Nota-se que as palavras baghdad e mosque são as mais citadas, pois são escritas com tamanho de fonte maior, seguidas pelas palavras attack, bombers, iraq, shiite e suicide.

Hassan-Montero e Herrero-Solana (Hassan-Montero e Herrero-Solana, 2006) propõem uma técnica baseada em Tag Cloud na qual as palavras importantes de uma coleção são agrupadas com base em suas relações de co-ocorrência. No entanto, a técnica gera muitos espaços vazios entre as palavras, desperdiçando espaço na visualização. De forma 


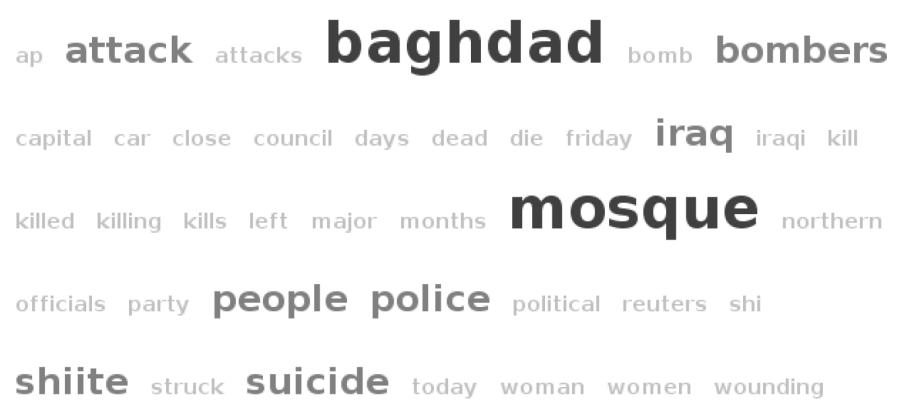

Figura 2.4: Visualização Tag Cloud para uma coleção de notícias, utilizando a ferramenta PEx (Paulovich et al., 2007).

contrária, Seifert et al. (Seifert et al., 2008) propõem uma técnica com o objetivo de otimizar ao máximo o espaço de visualização, eliminando espaços vazios entre as palavras. No entanto, para atingir esse objetivo a técnica pode eliminar algumas relações de coocorrência importantes entre as palavras.

Cui et al. (2010) propõem uma técnica de visualização para coleções de documentos dinâmicas baseada em Tag Cloud na qual, por meio da eliminação de espaços vazios, o espaço da representação da nuvem é otimizado. Além disso, a técnica preserva as relações de coerência semântica entre as palavras, i.e., relações de co-ocorrência, de importância ou de similaridade no significado.

A Figura 2.5 apresenta um exemplo de visualização gerada com a técnica de Cui et al. (2010). As Figuras 2.5a a 2.5e, descrevem cinco Tag Clouds de uma coleção de documentos sobre a empresa Apple, correspondentes a cinco intervalos de tempo diferentes. Por exemplo, a Figura 2.5a apresenta uma Tag Cloud dos documentos correspondentes ao mês de Maio de 1995, a Figura 2.5b exibe os documentos que correspondem ao mês de Outubro de 1998. Já na Figura 2.5f é apresentado um gráfico de frequência de uma das palavras da coleção, para análise da sua evolução ao longo do tempo.

A Tag Cloud gerada pelo sistema representa um conjunto de palavras importantes da coleção. Para extrair essas palavras, é necessário efetuar as seguintes tarefas: (i) primeiro, as palavras comuns e pouco importantes (stopwords) são removidas; (ii) em seguida, o algoritmo de lematização de Porter (Porter, 1997) é utilizado para reduzir as palavras restantes a seus radicais; (iii) a seguir é gerado um histograma de frequência das palavras restantes, sendo que aquelas palavras cujas frequências ultrapassarem o valor de um limiar, definido pelo usuário, serão incluídas no conjunto de palavras importantes. 


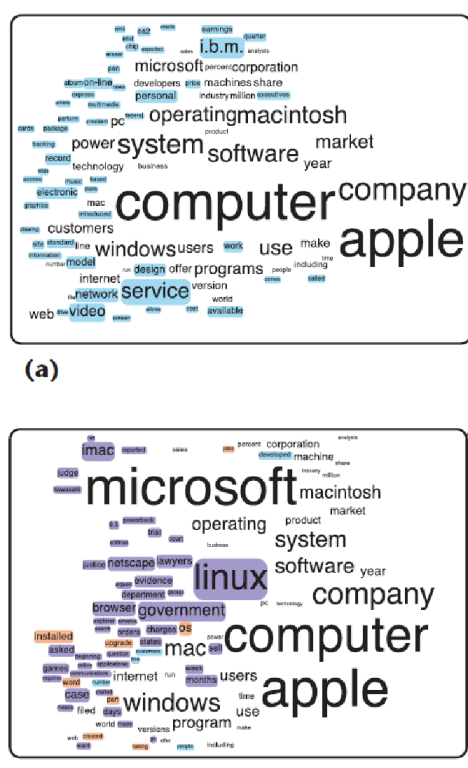

(b)

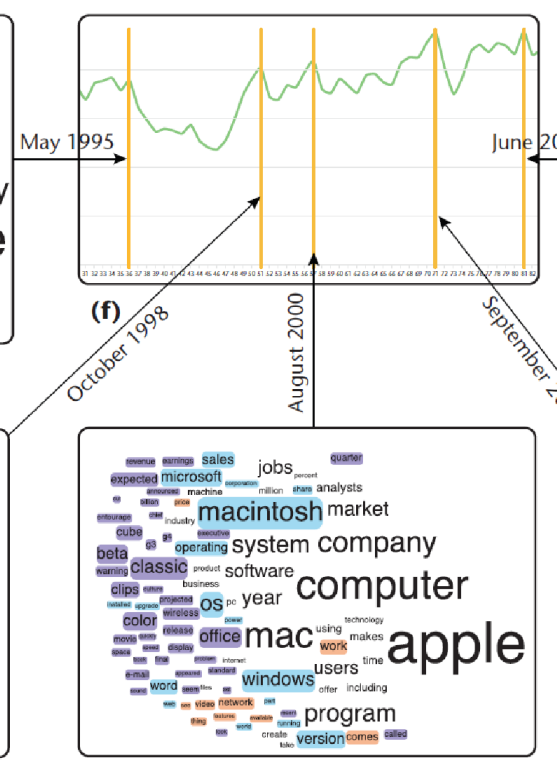

(c)

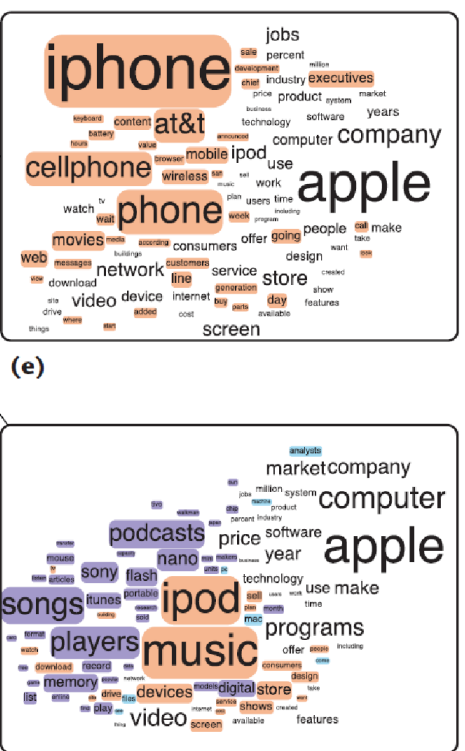

(d)

Figura 2.5: Representação gerada pela técnica de Cui et al. (2010) para uma coleção de documentos dinâmica. As Figuras 2.5a até 2.5e representam cinco nuvens de palavras construídas referentes a cinco diferentes intervalos de tempo. A Figura 2.5f apresenta um gráfico de frequência de uma palavra da coleção, para análise da sua evolução entre Maio de 1995 e Junho de 2007. Fonte Cui et al. (2010).

Após a extração do conjunto de palavras importantes, o algoritmo posiciona as palavras em um plano 2D, agrupando-as segundo um dos critérios de coerência semântica. Os autores estabelecem três critérios de coerência semântica:

- Critério de importância: com esse critério, a disposição das palavras no plano é definida pela sua importância. Assim, palavras com tamanho de fonte similar ficam próximas na visualização. Nesse contexto, um vetor de características $V_{i}=$ $\left\{v_{1}, v_{2}, \cdots, v_{i}\right\}$ com $i$ sendo o número de pontos de tempo da visualização, é gerado para cada palavra do conjunto. O elemento $v_{j}$ desse vector representa o valor de importância (tamanho da fonte) da palavra no instante de tempo $j$.

- Critério de co-ocorrência: utilizando esse critério, as palavras que são propensas a aparecer ou desaparecer de forma simultânea ficam próximas no plano de visualização. O vetor de características gerado para cada palavra da coleção é definido como $V_{a}=\left\{v_{1}, v_{2}, \cdots, v_{i}\right\}$ com $i$ sendo o instante de tempo da visualização. O elemento $v_{j}$ será 1 se a palavra é visível no instante de tempo $j$, caso contrário, o valor do elemento $v_{j}$ será 0 . 
- Critério de similaridade: utilizando esse critério, as palavras com significado semântico ${ }^{3}$ similar serão posicionadas próximas no plano da visualização. Neste caso, para uma palavra $p$, seu vetor de características estará definido por $V_{s}=$ $\left\{v_{1}, v_{2}, \cdots, v_{i}\right\}$ com $i$ sendo o tamanho do conjunto de palavras importantes. $\mathrm{O}$ elemento $v_{j}$ representa o número de vezes que apalavra $p$ aparece cerca da palavra $r(\forall r \in$ conjunto de palavras importantes). Esse valor é obtido utilizando o método de Hinrich Schütze (Schütze, 1998).

Escolhido um dos critérios anteriormente descritos, o cálculo da similaridade entre dois vetores pode ser definido utilizando a distância dos cossenos, criando assim uma matriz de dissimilaridade para todas as palavras do conjunto. Dessa maneira, uma técnica de escalonamento multidimensional (MDS) utilizará essa matriz para gerar a visualização desse conjunto de palavras no plano $2 \mathrm{D}$.

Já no passo seguinte, uma triangulação de Delaunay é aplicada sobre as posições das palavras no plano, obtendo uma malha de triângulos. A malha pode ser estruturada como um grafo, que dá a flexibilidade necessária para mover as posições das palavras guardando as relações semânticas entre elas. Para otimizar a utilização do espaço de visualização os autores utilizam um modelo de forças adaptado para Tag Cloud, assim espaços vazios são removidos e a topologia do grafo é mantida.

As Tag Clouds geradas pela técnica exibem as palavras importantes da coleção, e não os documentos. Quando novos documentos são agregados, a visualização deve ser refeita. O agrupamento semântico das palavras, além de melhorar a legibilidade da Tag Cloud, permite que novas visualizações mantenham uma estrutura similar à anterior, assim o usuário não perderá a familiaridade com a nuvem. Os autores não apresentam informação sobre o custo computacional.

ProjCloud (Paulovich et al., 2012) é outra técnica com o propósito de visualizar conjuntos de documentos utilizando Tag Cloud. Ao contrário dos métodos de visualização apresentadas anteriormente, a técnica de visualização ProjCloud mapeia os documentos de uma coleção, e não somente palavras. A metodologia combina projeções multidimensionais e um algoritmo para o posicionamento de palavras-chave dentro de polígonos. As Tag Clouds são construídas com base nas coordenadas resultantes da projeção multidimensional. Grupos de documentos posicionados próximos no espaço visual (semelhantes no conteúdo) serão encerrados dentro de polígonos, de forma manual ou automática, para definir as regiões em que as Tag Cloud serão construídas. Isso dá uma associação imediata

\footnotetext{
${ }^{3} \mathrm{O}$ termo semântico refere-se aos aspectos de significado, sentido ou interpretação do significado de uma palavra.
} 
entre as posições das palavras-chave e seus documentos base. Um exemplo da visualização gerada com esta técnica é apresentado na Figura 2.6.

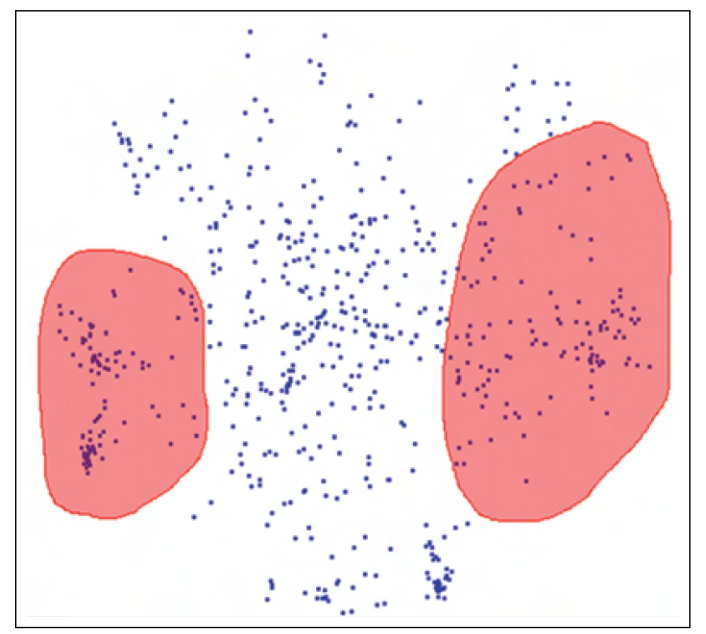

(a)

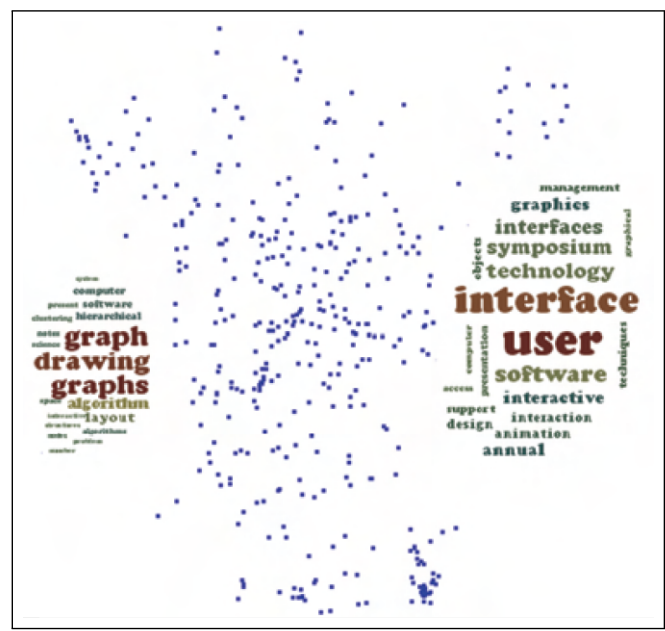

(b)

Figura 2.6: Visualização gerada com a ProjCloud: nuvens de palavras são geradas dos grupos identificados na projeção multidimensional. Fonte Paulovich et al. (2012)

O processo começa com a construção de um mapa visual da coleção de documentos utilizando alguma técnica de projeção multidimensional, como a LSP Paulovich et al. (2008). Para isso, documentos são pré-processados e uma representação VSM é gerada, a qual servirá como entrada para a LSP. Gerada a projeção, a identificação de grupos de documentos pode ser efetuada de duas formas: (i) manualmente (o usuário pode interativamente desenhar uma região (polígono) contendo um subconjunto de documentos de interesse) ou (ii) automaticamente. Neste último caso, pontos no espaço visual são agrupados utilizando o algoritmo bisecting k-means (Steinbach et al., 2000) para posteriormente computar o fecho convexo (convex hull) de cada agrupamento, dessa maneria os dados são segmentados em regiões convexas sem sobreposição. Na etapa seguinte, palavras-chaves são determinadas a partir das palavras mais frequentes relacionadas aos documentos contidos em cada polígono. A fim de conduzir o posicionamento das palavraschave preservando as relações semânticas, a relevância de cada palavra-chave é computada utilizando uma abordagem que organiza na ordem crescente as palavras-chave de acordo com sua importância e dentro de um vetor, removendo as de baixa relevância. Este vetor é utilizado pela ProjCloud para estabelecer as posições das palavras-chave dentro da nuvem. O tamanho da fonte é dimensionado com base na relevância da palavra-chave e de acordo com a área do polígono que a contém. 
Finalmente, as palavras-chave são dispostas no interior dos polígonos usando um algoritmo de otimização. Para isso, palavras-chaves são substituídas pelas suas correspondentes caixas delimitadoras. Dessa forma, uma variante do Cutting Stock Problem (CSP) (Wäscher et al., 2007) no espaço bidimensional pode ser utilizada para resolver o problema de enchimento de um polígono com caixas retangulares de tamanhos distintos. CSP é um clássico problema de otimização combinatória para potencializar o processo de corte de peças grandes para a produção de peças menores (itens) de distintos tamanhos, em quantidades especificadas, possibilitando rotações de $90^{\circ}$.

\subsection{Incremental Board}

Pinho et al. (2009) desenvolveram a técnica de visualização Incremental Board (incBoard). A técnica é baseada na visualização dos dados multidimensionais por meio da sua disposição em um tabuleiro, análogo a um tabuleiro de xadrez, que ocupa o espaço bidimensional dividido em células dispostas em linhas e colunas. Em (Pinho et al., 2010) se propõe a geração de um layout (incSpace) baseado nas semelhanças entre elementos, e sem considerar a metáfora do tabuleiro de xadrez para a disposição dos elementos no espaço visual.

Dois estudos de caso são apresentados: no primeiro cenário, o algoritmo é aplicado a coleções de documentos dinâmicas, como conjuntos de notícias, artigos, etc. Já uma segunda aplicação é para exibir coleções de imagens. Nessa última, a similaridade é computada usando o coeficiente de Jaccard sobre um conjunto de etiquetas que descrevem os objetos e características nas imagens.

O cálculo do VSM de uma coleção requer que todos os documentos estejam disponíveis a priori, a adição de um novo documento à coleção forçaria o recálculo das similaridades previamente computadas. Neste sentido, Pinho et al. (2010) propõem um modelo vetorial modificado para representar os textos de uma coleção incremental (ver Seção 3.2.2), aqui denominado iVSM ou incremental VSM. O cálculo do modelo vetorial incremental é feito para cada documento de uma sequência, um a um, de forma incremental. O iVSM utiliza como ponto de partida um modelo de linguagem, o qual é gerado a partir de um modelo vetorial construído para uma coleção similar já disponível. A similaridade entre documentos é medida utilizando a distância do cosseno. Termos frequentes (stopwords) são removidos e cortes de Luhn são aplicados para a seleção de termos relevantes. No Capítulo 6, realizamos uma comparação entre o VSM convencional o modelo vetorial modificado e investigamos como este se comporta na geração de mapas visuais baseados no posicionamento de pontos. 
No incBoard, para posicionar os elementos da coleção de dados multidimensional no plano, cada elemento $E_{i}$ da coleção é representado no espaço visual por um ponto $p_{i}$ com as coordenadas $\left(x_{i} ; y_{i}\right)$, com $x_{i}, y_{i} \in \mathbb{Z}$. Para o cálculo das distâncias de uma célula aos seus vizinhos imediatos, é usada a distância de Chebyshev, segundo a Equação 2.1:

$$
d c\left(E_{i}, E_{j}\right)=\max \left|x_{i}-x_{j}\right|,\left|y_{i}-y_{j}\right|
$$

Essa distância mede o número de passos necessários para que um elemento seja movido de uma célula a outra. O benefício de utilizar a distância de Chebyshev está na obtenção de um comportamento mais consistente quando são computadas as distâncias de uma célula aos seus vizinhos imediatos, além de produzir um mapa com melhor aproveitamento do espaço visual.

A técnica adotada busca posicionar os elementos no tabuleiro de forma que para cada elemento $E_{i}$, o ranking obtido pela similaridade deste elemento $E_{i}$ em relação aos outros elementos no espaço original seja o mais similar possível ao ranking obtido pela distância de Chebyshev, $d_{c}$, da localização desse elemento em relação à localização dos outros elementos no tabuleiro. Então, para cada elemento $E_{i}$ é calculado um erro $W_{\text {err }}\left(E_{i}, L\right)$ que indica o quanto os rankings no espaço original e no espaço bidimensional do tabuleiro concordam ou não.

Assim, para uma lista de elementos $L$, o erro $W_{e r r}\left(E_{i}, L\right)$ para um elemento $E_{i}$ é calculado por:

$$
W_{e r r}\left(E_{i}, L\right)=\sum_{E_{j} \in L}\left|R_{c i}\left(E_{j}\right)-R_{n i}\left(E_{j}\right)\right| \times\left(|L|-R_{n i}\left(E_{j}\right)\right)
$$

sendo que $R_{c i}$ representa a posição esperada e $R_{n i}$ a posição efetiva. A lista $L$ pode ser composta de dois modos: modo completo, quando são considerados todos os elementos atualmente presentes no tabuleiro; ou modo estocástico, quando a lista é obtida aleatoriamente. O modo completo apresenta melhores resultados quando o mapa tem um número pequeno de elementos, ou para conjunto de dados que sofrem alterações com pouca frequência, mas é computacionalmente caro. Assim, a técnica utiliza o modo completo para os primeiros elementos, passando logo ao modo estocástico. Dessa maneira, o processo estocástico opera sobre uma configuração mais estável do tabuleiro, em que a escolha aleatória dos elementos para compor a lista $L$ não altera significativamente a qualidade do resultado, mantendo o custo computacional baixo. 
O espaço incremental sempre está em um de dois estados: (1) estável, quando cada célula tem só um elemento; (2) instável, quando uma célula tem mais de um elemento. Quando o estado é instável é preciso uma intervenção para retornar a estabilidade. Para isso, dezesseis soluções são avaliadas, oito delas mantêm o novo elemento e move o elemento mais antigo para uma das oito células vizinhas imediatas. As oito soluções restantes mantêm a posição do elemento mais antigo e movem o elemento mais novo. A opção que possuir o menor erro para ambos os elementos é escolhida. No entanto, este processo pode gerar uma configuração em que outra célula tem dois elementos. Neste caso, o processo é repetido para essa nova célula até que nenhuma célula contenha dois elementos e termine com uma célula vazia sendo ocupada. Para evitar ciclos, a técnica mantém uma lista das células já visitadas.

Todas as operações necessárias à adição ou remoção de elementos consideram apenas os elementos no tabuleiro. Portanto, um conjunto completo de dados de tamanho $c$ pode ser exibido sequencialmente como um custo computacional constante para cada item adicionado ou removido, desde que seja mantida uma janela de visualização de tamanho fixo $J$. A complexidade algorítmica para a exibição de todo o conjunto de dados seria $O(J \times c)$ ou simplesmente $O(c)$, mantendo-se $J$ constante. Se a coleção completa é apresentada simultaneamente, o custo computacional pode atingir $O\left(c^{2}\right)$. Esta técnica resulta em uma visualização sem problemas de oclusão. Além disso, a identificação de áreas temáticas é alcançada com técnicas de extração de regras de associação representativas para a identificação automática de tópicos.

A Figura 2.7 mostra estágios intermediários e final de um mapa em que documentos (o mesmo conjunto CBR-ILP-IR já citado) foram coloridos de acordo com suas classes atribuídas. Observa-se que a disposição geral das classes mantém-se globalmente estável à medida que o mapa é construído.

\subsection{Considerações Finais}

Neste capítulo foram apresentadas diferentes técnicas para visualizar coleções de documentos dinâmicas. Tipicamente, os documentos são representados utilizando o VSM, no qual mede-se a frequência das palavras na coleção. Além disso, o cálculo de similaridade entre documentos é computado usando a distância dos cossenos. (Wei et al., 2010) mostraram que técnicas de extração de tópicos também são utilizadas para construir mapas visuais a partir de um conjunto de palavras-chave que representam de forma global o conteúdo de uma coleção de textos. 
Quando novos documentos são inseridos, ou documentos já existentes são removidos, a disposição dos elementos no espaço de visualização é ajustada segundo o re-cálculo das novas distâncias entre os documentos presentes na visualização. Entretanto, isso pode gerar efeitos indesejáveis para o usuário, que pode ficar perdido se todos os documentos do mapa foram reposicionados. Com esse objetivo, a técnica apresentada por Pinho et al. (2010) sugere a utilização de um modelo global de linguagem para a construção do um modelo vetorial modificado, de forma que a adição de novos documentos não afete as similaridades anteriormente calculadas.

No próximo capítulo, detalhes da construção do VSM e do modelo vetorial modificado para representar coleções de textos incrementais, são apresentadas. Também são estudadas diferentes métricas para computar a similaridade entre dois documentos. 


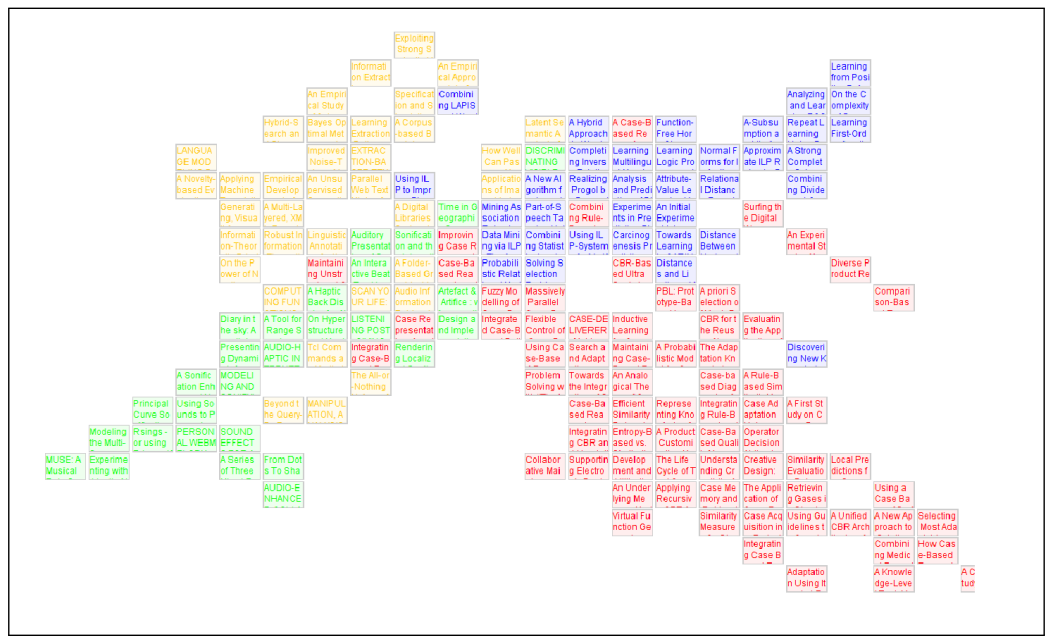

(a) 178 documentos

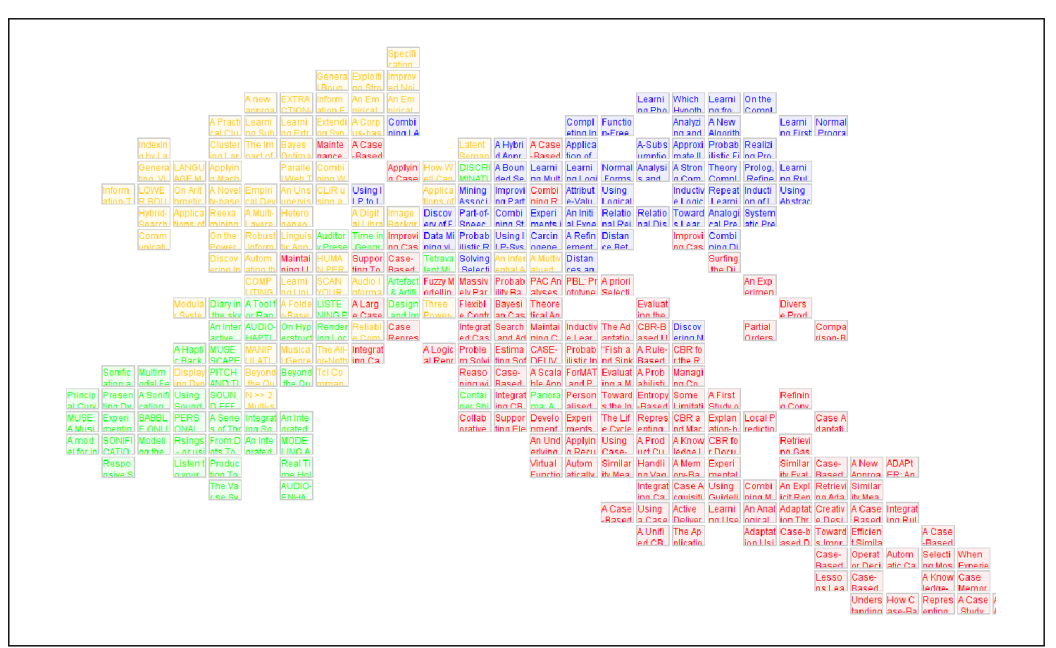

(b) 294 documentos

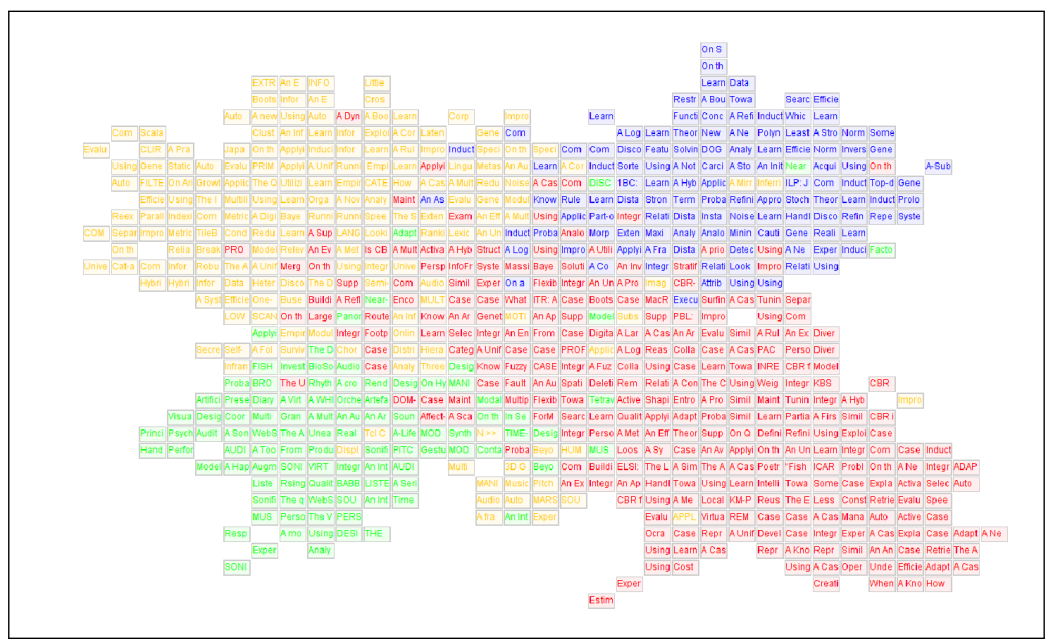

(c) 675 documentos

Figura 2.7: Três momentos na construção incremental do mapa de um corpus dinâmico de documentos utilizando a técnica incBoard. As cores representam as classes dos documentos. Fonte (Pinho, 2009). 


\section{Cálculo de Dissimilaridade entre Documentos}

\subsection{Considerações Iniciais}

Documentos tipicamente necessitam de uma representação que possibilite a sua mineração, recuperação e visualização. Tal representação deveria descrever a informação originalmente contida com a mínima perda de informação possível. Assim, uma representação adequada da coleção é de suma importância para a qualidade dos resultados das atividades posteriores. Para as visualizações por posicionamento de pontos segundo a (dis)similaridade, é importante ter uma boa estimativa de semelhança. Isto pode ser feito, utilizando diferentes métricas, de duas maneiras: a partir de uma representação gerada a partir do conteúdo dos documentos, ou diretamente comparando strings textuais, sem adotar uma representação intermediária.

Neste capítulo, na Seção 3.2 são apresentados métodos para a representação de documentos. De posse de uma representação da coleção, é possível utilizar medidas de dissimilaridade sobre essas representações para quantificar a semelhança de conteúdo entre dois documentos. Na Seção 3.3 são apresentadas diversas métricas para computar a similaridade entre textos, considerando duas abordagens: as que operam sobre os modelos vetoriais e as que operam diretamente sobre as strings. 


\subsection{Métodos de Representação de Textos}

Na literatura observa-se que o VSM é uma das abordagens tipicamente adotadas para representar textos (Cuadros et al., 2007; Lopes et al., 2006; Minghim et al., 2006; Paulovich e Minghim, 2006; Paulovich et al., 2012). No entanto, a VSM convencional não é incremental, ou seja, novas adições à coleção forçam o recálculo do espaço e das dissimilaridades. Nesse sentido, Pinho (2009) introduz uma variação incremental do VSM. Detalhes sobre a construção desse novo modelo são descritos na Seção 3.2.2. Observase que estes modelos de representação não são únicos, pois existem vários parâmetros e procedimentos que podem ser aplicados ou não, alterando o resultado.

\subsubsection{Modelo Vetorial}

O modelo vetorial, ou Modelo do Espaço Vetorial, do inglês Vector Space Model (VSM) (Salton et al., 1975), é uma abordagem que representa os documentos de uma coleção como vetores em um espaço multidimensional. Cada uma das dimensões desses vetores representa a ocorrência, ou frequência, de uma palavra, elemento ou termo presente nos documentos correspondentes. Esta representação é ilustrada na Tabela 3.1 para uma coleção de $N$ documentos $d=\left(d_{1}, d_{2}, \ldots, d_{N}\right)$ que inclui $M$ elementos diferentes $t=\left(t_{1}, t_{2}, \ldots, t_{M}\right)$. Cada documento $d_{i}$ é um vetor $d_{i}=\left(\alpha_{i 1}, \alpha_{i 2}, \ldots, \alpha_{i M}\right)$, no qual o valor $\alpha_{i j}$ é uma medida de ocorrência, ou frequência, do termo $t_{j}$ no documento $d_{i}$.

\begin{tabular}{|c|c|c|c|c|}
\hline & $t_{1}$ & $t_{2}$ & $\ldots$ & $t_{M}$ \\
\hline \hline$d_{1}$ & $\alpha_{11}$ & $\alpha_{12}$ & $\ldots$ & $\alpha_{1 M}$ \\
\hline$d_{2}$ & $\alpha_{21}$ & $\alpha_{22}$ & $\ldots$ & $\alpha_{2 M}$ \\
\hline$\ldots$ & $\ldots$ & $\ldots$ & $\ldots$ & $\ldots$ \\
\hline$d_{N}$ & $\alpha_{N 1}$ & $\alpha_{N 2}$ & $\ldots$ & $\alpha_{N M}$ \\
\hline
\end{tabular}

Tabela 3.1: Representação do modelo de espaço vetorial de uma coleção hipotética de documentos. Cada linha representa um documento e cada coluna representa um termo no documento. As entradas na tabela indicam a frequência de um termo em um determinado documento.

O valor $\alpha_{i j}$ pode ser calculado de várias formas. Algumas das mais utilizadas são descritas a seguir: 
- Booleana: Esta medida indica se o termo $t_{j}$ faz parte do documento $d_{i}$.

$$
\alpha_{i, j}= \begin{cases}1, & \text { se } t_{j} \in d_{i} \\ 0, & \text { caso contrário }\end{cases}
$$

- Frequência do termo (term frequency - $t f)$ : Esta medida é o número de vezes que o termo $t_{j}$ ocorre no documento $d_{i}$.

$$
\alpha_{i j}=t f\left(t_{j}, d_{i}\right)=f r e q\left(t_{j}, d_{i}\right)
$$

- Frequência do termo inverso da frequência do documento (term frequency inverse document frequency - tf-idf (Salton et al., 1975)): Nesta medida, a importância de um termo é proporcional à sua frequência de ocorrência em cada documento da coleção, e inversamente proporcional ao número de documentos em que ele aparece.

$$
\alpha_{i j}=t f-i d f\left(t_{j}, d_{i}\right)=t f\left(t_{j}, d_{i}\right) * \log _{2} \frac{N}{d f\left(t_{j}\right)}
$$

onde $N$ é o número de documentos e $d f\left(t_{j}\right)$ é o número de documentos que contém o termo $t_{j}\left(t_{j} \neq 0\right)$.

O vetor que representa cada documento contém, em princípio, todos os termos utilizados em todos os documentos da coleção. Assim, quanto maior é a coleção, maior será, provavelmente, a dimensionalidade do espaço vetorial que a representa. A alta dimensionalidade aumenta o custo de processamento. Assim, são apresentadas algumas das estratégias de pré-processamento para melhorar a qualidade da representação vetorial e reduzir a dimensionalidade do espaço resultante.

- Remoção de termos irrelevantes (stopwords)- Termos complementares como artigos, preposições, pronomes e conjunções (Baeza-Yates e Ribeiro-Neto, 1999) são filtradas e eliminadas. Esses termos são dependentes da língua adotada no documento, assim um conjunto de termos irrelevantes apresentado para um documento em português (Wilbur e Sirotkin, 1992) é diferente do utilizado para um documento em inglês (Fox, 1989).

- Redução dos termos a seus radicais (stemming)- As variantes de um termo são semanticamente equivalentes, e podem ser tratadas como um único termo. Assim, os radicais dos termos devem ser identificados e os sufixos e prefixos removidos. Por exemplo, os termos (eleição, eleições, eleitorais, eleitor) são similares e podem ser 
reduzidas ao radical elei. O algoritmo de Porter (Porter, 1997), por exemplo, faz essa redução para termos em inglês.

- Lei de Zipf e aplicação de cortes superiores e inferiores de Luhn- Outra forma de reduzir a dimensionalidade da representação é procurar por termos pouco representativos. A Lei de Zipf (Zipf, 1949) baseia-se na frequência dos termos para encontrar os menos representativos. Considerando um histograma de frequências dos termos de uma coleção de documentos, ordenada de forma decrescente e de acordo com as frequências, obtém-se a "Curva de Zipf". Se o gráfico dessa curva for apresentado na escala logarítmica, a curva será semelhante à apresentada na Figura 3.1(a). Luhn (Luhn, 1958) usou a curva para especificar dois limiares (superior e inferior) para remover termos pouco representativos, ver Figura 3.1(b). Os termos cuja frequência está acima do limiar superior são considerados pouco representativos por serem muito comuns. Já os termos com frequência abaixo do limiar inferior também são considerados pouco representativos por serem raros e não contribuir na discriminação dos documentos. Os valores dos cortes de Luhn são estabelecidos de forma empírica, pois não existe uma base teórica para sua determinação. A Figura 3.1 ilustra os cortes de Luhn sobre uma curva de Zipf com a frequência $(t f-i d f)$ dos termos significativos de um corpus que contém 574 artigos científicos.

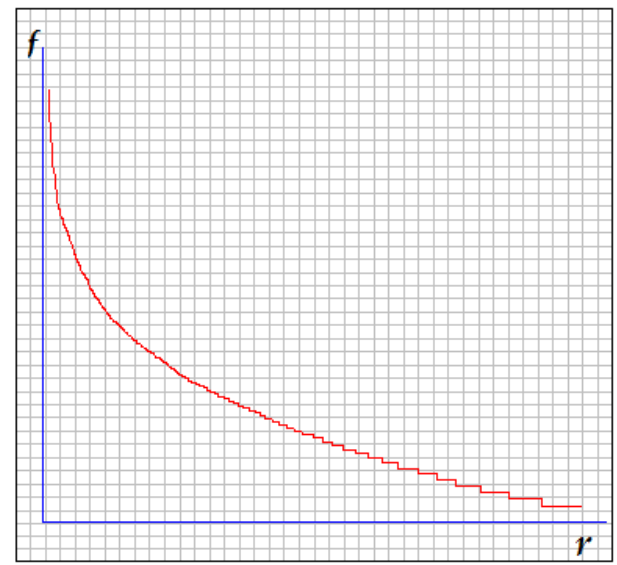

(a) Curva de Zipf

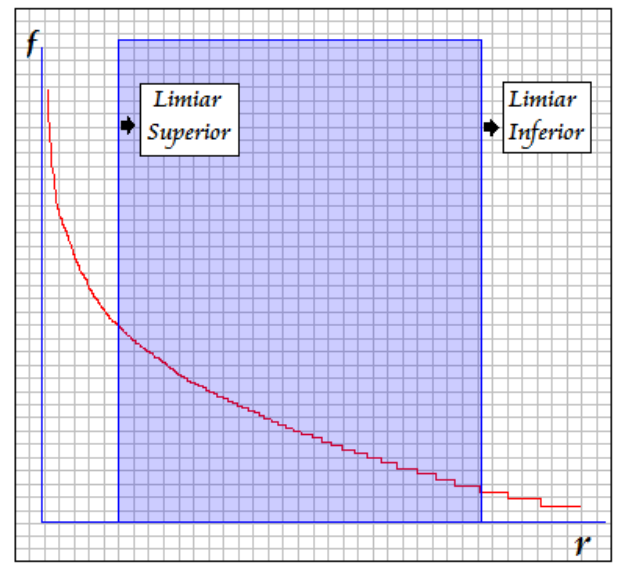

(b) Cortes de Lunh

Figura 3.1: Cortes de Luhn aplicados a uma curva de Zipf para uma coleção de artigos científicos, gerada com a plataforma Projection Explorer (PEx) (Paulovich et al., 2007). O eixo cartesiano $r$ representa os termos da coleção e o eixo cartesiano $f$ representa a frequência desses termos. 


\subsubsection{Modelo Vetorial Incremental}

O Modelo Vetorial Incremental, ou Incremental Vector Space Model (iVSM), é uma abordagem proposta para representar os textos de uma coleção incremental como vetores em um espaço multidimensional (Pinho, 2009). Cada uma das coordenadas destes vetores representa a medida $t f-i d f$ da frequência de um termo relevante na coleção.

A abordagem proposta utiliza como ponto de partida um modelo de linguagem, gerado a partir de um modelo vetorial construído para uma coleção de documentos já disponível. Esse modelo de linguagem contém os termos relevantes da coleção conhecida, a sua frequência $(T F)$ e o número de documentos nos quais o termo aparece $(D F)$. A seguir descrevemos como o modelo de linguagem é gerado, em seguida o procedimento de construção do modelo vetorial incremental.

\section{Construção do Modelo de Linguagem}

Para explicar a abordagem, consideremos que a Tabela 3.1 representa o modelo vetorial para uma coleção hipotética de $N$ documentos. O modelo de linguagem para essa coleção é definido como segue:

\begin{tabular}{|c|c|c|}
\hline Termo & $T F$ & $D F$ \\
\hline \hline$t_{1}$ & $T F_{1}$ & $D F_{1}$ \\
\hline$t_{2}$ & $T F_{2}$ & $D F_{2}$ \\
\hline$\ldots$ & $\ldots$ & $\ldots$ \\
\hline$t_{M}$ & $T F_{M}$ & $D F_{M}$ \\
\hline
\end{tabular}

Tabela 3.2: Representação do modelo de linguagem de uma coleção de documentos hipotética. Cada linha representa um termo no modelo vetorial, a coluna $T F$ representa a frequência do termo na coleção e, a coluna $D F$ a quantidade de documentos da coleção nos quais o termo aparece.

$D F_{j}$ é o número de documentos no qual o termo $j$ aparece e $T F_{j}$ é a frequência do termo $j$, calculada utilizando a Equação 3.4:

$$
T F_{j}=\frac{\sum_{i=1}^{N} \alpha_{i j}}{\log \frac{N}{D F_{j}}}
$$

Esse modelo de linguagem será utilizado para gerar o modelo vetorial incremental de outras coleções de documentos similares. 


\section{Geração do Modelo Vetorial Incremental}

O cálculo do modelo vetorial incremental é feito para cada documento de uma sequência, um a um, de forma incremental. Assim, com a adição de cada novo documento o modelo de linguagem é atualizado, considerando cada termo relevante nele contido.

A ideia é simples: se um termo presente no documento está também presente no modelo de linguagem, então incrementamos de 1 o valor de $T F$, e também, se for a primeira vez que o termo aparece no documento, o valor $D F$ do termo no modelo. Caso contrário, adicionamos o novo termo ao modelo e inicializamos os valores de $T F$ e $D F: T F=1$, $D F=1$.

Devido à alta dimensionalidade do modelo de linguagem, que aumenta na medida em que ele é atualizado, aplicamos os cortes inferior $\left(C L_{\text {inferior }}\right)$ e superior $\left(C L_{\text {superior }}\right)$ de Luhn. Os valores dos limiares são automaticamente atualizados a cada documento acrescentado, de acordo com as seguintes fórmulas:

$$
\begin{aligned}
& C L_{\text {inferior }}= \begin{cases}3, & \text { se } C L_{\text {inferior }}<3 \\
c l i \text { de } N^{\prime}, & \text { se } C L_{\text {inferior }}>5 \% \text { de } N^{\prime} \\
& \text { ou } C L_{\text {inferior }}<1 \% \text { de } N^{\prime}\end{cases} \\
& C L_{\text {superior }}=\left\{\begin{array}{cl}
c l s \text { de } N^{\prime}, & \text { se } C L_{\text {superior }}<90 \% \text { de } N^{\prime} \\
& \text { ou } C L_{\text {superior }}>N^{\prime}
\end{array}\right.
\end{aligned}
$$

com cli e $c l s$ como os limiares dos cortes inferior e superior de Luhn, respectivamente.

Seja $N^{\prime}=\max \left(D F_{i}\right)$ para $i=\{1, \ldots, M\}$ no modelo de linguagem atualizado. Finalmente, o cálculo do modelo vetorial incremental para um documento utiliza a contagem de frequência $t f-i d f$ de cada termo $\left(t_{j}\right)$ presente no modelo de linguagem, segundo a Equação 3.7, com $t f_{i j}$ sendo a quantidade de vezes que o termo $j$ aparece no documento $i$ :

$$
i V S M_{i j}= \begin{cases}t f_{i j} * \log \frac{N^{\prime}}{D F_{j}}, & \text { se } D F_{j} \geq C L_{\text {inferior }} \\ & \text { ou } D F_{j} \leq C L_{\text {superior }} \\ \text { zero, } & \text { caso contrário }\end{cases}
$$

\subsection{Medidas de Dissimilaridade entre Documentos}

De posse de uma representação da coleção de documentos, como um modelo vetorial, é possível utilizar medidas de dissimilaridade para quantificar a semelhança de conteúdo 
entre dois documentos. Uma primeira possibilidade são medidas de distância aplicáveis a espaços vetoriais, como a distância Euclidiana, que representa a noção intuitiva de distância nos eixos cartesianos, e a similaridade do Cosseno, entre dois vetores, empregada para medir distância em conjuntos de dados esparsos.

- A medida da distância Euclidiana entre dois documentos $d_{1}$ e $d_{2}$, representados por um vetor de atributos, é calculada pela seguinte equação:

$$
\operatorname{dist}_{\text {euclidiana }}\left(d_{1}, d_{2}\right)=\sqrt{\sum_{i=1}^{M}\left(\alpha_{1 i}-\alpha_{2 i}\right)^{2}}
$$

onde $M$ é o número de termos da representação ou a dimensionalidade do espaço vetorial, e $\alpha_{i j}$ é a frequência do termo $t_{j}$ no documento $d_{i}$. Assim, quanto mais próximo de zero for o valor da distância, mais similares são os documentos $d_{1}$ e $d_{2}$. No entanto, essa métrica não é adequada para calcular a distância entre documentos, em que muitos dos atributos do vetor têm valor zero. Assim, a métrica pode considerar as comparações do tipo zero-zero (que não agregam informação), sendo que a representação é esparsa, e alguns documentos serão indicados como similares pelo número de termos que ambos não possuem.

- A distância Manhattan, conhecida também como métrica do táxi, distância $L_{1}$ ou distância city block, tem como principal beneficio a sua simplicidade e rapidez no momento de realizar os cálculos. Se $d_{1}$ e $d_{2}$ são dois documentos representados por $M$ termos, a distância de Manhattan é calculada pela Equação 3.9:

$$
\operatorname{dist}_{\text {manhattan }}\left(d_{1}, d_{2}\right)=\sum_{i=1}^{M}\left|\alpha_{1 i}-\alpha_{2 i}\right|
$$

quanto mais próximo de zero for o valor da distância, mais similares são os documentos $d_{1}$ e $d_{2}$. Esta métrica não é comumente utilizada para calcular a similaridade entre documentos.

- A similaridade do Cosseno é uma das medidas mais utilizadas para definir a similaridade entre dois documentos, pois favorece comparações do tipo zero-zero, sendo uma medida indicada justamente para espaços de alta dimensionalidade e esparsos. O princípio dessa métrica é medir o ângulo formado por dois vetores 
como uma aproximação de sua similaridade. A similaridade do Cosseno é calculada pela Equação 3.10:

$$
\operatorname{sim}_{\text {cosseno }}\left(d_{1}, d_{2}\right)=\frac{\sum_{i=1}^{M}\left(\alpha_{1 i} \times \alpha_{2 i}\right)}{\sqrt{\sum_{i=1}^{M} \alpha_{1 i}^{2}} \times \sqrt{\sum_{i=1}^{M} \alpha_{2 i}^{2}}},
$$

Essa medida retorna valores no intervalo $[0,1]$. Conforme o ângulo entre os vetores diminui, o cosseno do ângulo se aproxima de 1, indicando que sua distância é menor. Para transformar essa medida em uma medida de dissimilaridade, ou distância, basta utilizar dist cosseno $=1-\operatorname{sim}_{\text {cosseno }}\left(d_{1}, d_{2}\right)$.

Uma segunda alternativa para coleções de documentos é aplicar uma medida de dissimilaridade não baseada em vetores. A definição da similaridade entre dois documentos pode ser calculada de forma direta, sem gerar uma representação vetorial, aplicando operações simples sobre o tamanho compacto dos documentos.

Neste momento, é necessário definir alguns termos, o que é feito a seguir:

Definição 3.1 (Alfabeto) Um alfabeto $\Sigma$ é um conjunto finito de caracteres ou letras.

Definição 3.2 (String) Toda sequência formada sobre caracteres de um alfabeto $\Sigma$ é uma string sobre $\Sigma$, ou simplesmente string quando o alfabeto for óbvio ou irrelevante. Exemplos de strings são:

- Os termos não acentuados da língua portuguesa são strings sobre o alfabeto de 26 letras;

- "1234" e "01001" são strings sobre o alfabeto $\{0,1, \ldots, 9\}$.

Denotamos o comprimento de uma string $X=\left\{x_{1} \ldots x_{c}\right\}$ por $|X|=c$. Uma string de comprimento zero é dita vazia. Denotamos também por $x_{i}$ o $i$-ésimo caractere de $X$. $O$ conjunto de todas as strings formadas sobre um alfabeto $\Sigma$ é denotado por $\Sigma^{*}$

Definição 3.3 (Métrica) Uma função distância, ou métrica, em $\Sigma^{*}$ é uma função do tipo dist : $\Sigma^{*} \times \Sigma^{*} \rightarrow \mathbb{R}$ para a qual valem as propriedades a seguir, para quaisquer strings $X, Y, Z \in \Sigma^{*}:$

$$
\begin{gathered}
\operatorname{dist}(X, Y)=\operatorname{dist}(Y, X) ; \\
\operatorname{dist}(X, X)=0
\end{gathered}
$$




$$
\begin{gathered}
X \neq Y \Leftrightarrow \operatorname{dist}(X, Y)>0 \\
\operatorname{dist}(X, Y)+\operatorname{dist}(Y, Z) \geq \operatorname{dist}(X, Z) .
\end{gathered}
$$

Consideramos também a função $\beta(x, y)$ que indica se dois caracteres, $x$ e $y \in \Sigma$, são iguais ou não:

$$
\beta(x, y)=\left\{\begin{array}{ll}
0 & \text { se } x=y \\
1 & \text { se } x \neq y
\end{array}, \forall x, y \in \Sigma\right.
$$

A função de distância $\operatorname{dist}(X, Y)$ retorna o menor custo possível associado às operações de edição necessárias para transformar $X$ em $Y$. As operações são nada mais do que um conjunto finito de regras no formato $\operatorname{dist}(X, Y)=z$, onde $X, Y \in \Sigma, X \neq Y$ e $z \in \mathbb{R}$. As quatro operações seguintes podem ser consideradas básicas:

- Inserção de um caractere ausente na string.

- Exclusão de um caractere presente na string.

- Substituição de um caractere presente na string por outro caractere.

- Transposição ou troca da posição de dois caracteres presentes na string.

Nem todas as métricas de similaridade/dissimilaridade entre strings trabalham com todas as operações acima descritas. De fato, pode-se simular uma operação de substituição como uma inserção seguida de uma exclusão; assim como pode-se simular uma operação de transposição como duas operações de substituição consecutivas. É claro que, uma vez que são necessárias duas ou mais operações para simular uma única operação, o custo associado é diferente e, dependendo da métrica utilizada, os resultados também podem ser diferentes. O valor da função de distância é dado pela soma dos custos individuais das operações utilizadas pela função. Exemplos de métricas utilizadas para medir a similaridade/dissimilaridade são apresentadas a seguir:

- A distância de Hamming (Sankoff e Kruskal, 1983) é utilizada para medir distâncias entre duas strings com igual comprimento. Permite somente operações de substituição, às quais é atribuído um custo fixo igual a 1. A função é definida pela 
seguinte recorrência:

$$
\operatorname{dist}_{H a m}\left(X_{1 \ldots c}, Y_{1 \ldots s}\right)= \begin{cases}0 & \text { se } c=0 \text { e } s=0 \\ \operatorname{dist}_{\text {Ham }}\left(X_{1 \ldots c-1}, Y_{1 \ldots s-1}\right)+ & \\ \beta\left(x_{c}, y_{s}\right) & \text { se } c=s \text { e } c, s>0 \\ \infty & \text { se } c \neq s\end{cases}
$$

- A distância de Levenshtein (Levenshtein, 1965, 1966), ao contrário da distância de Hamming, pode medir a distância entre duas strings com tamanhos diferentes. Permite operações de inserção, exclusão e substituição, atribuindo a todas o custo 1, independente da operação e dos caracteres envolvidos. A distância dist $_{\text {Levenshtein }}(X, Y)$ retorna o número mínimo de inserções, exclusões e substituições necessárias para tornar $X=Y$. A função é definida pela seguinte recorrência:

$$
\operatorname{dist}_{L e v}\left(X_{1 \ldots c}, Y_{1 \ldots s}\right)= \begin{cases}\max \{c, s\} & \text { se } c=0 \\
\min \left\{\begin{array}{c}
\operatorname{dist}_{\text {Lev }}\left(X_{1 \ldots c-1}, Y_{1 \ldots s-1}\right)+\beta\left(x_{c}, y_{s}\right) \\
\operatorname{dist}_{\text {Lev }}\left(X_{1 \ldots c}, Y_{1 \ldots s-1}\right)+1 \\
\operatorname{dist}_{\text {Lev }}\left(X_{1 \ldots c-1}, Y_{1 \ldots s}\right)+1
\end{array}\right\} & \text { se } c, s>0\end{cases}
$$

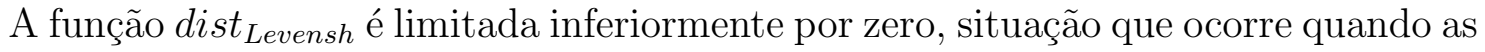
sequências de entrada são iguais, e superiormente pelo tamanho da maior sequência do domínio.

- A distância de Levenshtein Generalizada, assim como a distância de Levenshtein simples, permite apenas as operações de inserção, exclusão e substituição. No entanto, o custo das operações pode ser definido de maneira menos restrita, podendo ser qualquer número real não negativo. Além disso, diferentes operações com diferentes elementos podem ter custos específicos atribuídos. Por fim, ao contrário da distância de Levenshtein simples, qualquer função pode ser utilizada para agregar o custo individual das operações e retornar o valor da distância para a função $d_{\text {LevenshG }}$.

- A distância Episódica (Das et al., 1997) permite apenas operações de inserção, todas com custo 1. É utilizada em problemas que requerem encontrar a sucessão correta de uma série de eventos ocorridos em um período de tempo relativamente 
curto. Essa métrica de distância não é simétrica e existem casos em que é possível transformar $X$ em $Y$ (na realidade, só é possível transformar $X$ em $Y$ quando $X$ é uma subsequência de $Y)$. Dessa forma, $d_{\text {episodica }}(X, Y)=(|X|-|Y|)$ ou $\infty$.

- A distância da Subsequência Comum Mais Longa (Needleman e Wunsch, 1970) permite as operações de inserção, exclusão e substituição, atribuindo a todas custo 1. Como o nome indica, ela mede o comprimento da maior subsequência coincidente dos elementos entre $X$ e $Y$, respeitando a posição dos elementos. A distância $d_{S C M L}$ indica o número de elementos não coincidentes. A distância é simétrica e limitada inferiormente por zero (quando as sequências de entrada são iguais) e superiormente pela soma dos tamanhos de $X$ e $Y$.

- A distância de Smith-Waterman (Smith e Waterman, 1981) permite apenas as operações de inserção e exclusão de comprimento arbitrário. A distância determina as regiões semelhantes entre duas strings, encontrando um par de segmentos, um de cada uma das duas strings, de tal forma que não há nenhum outro par de segmentos com maior similaridade (homologia). Para exclusões de comprimento $k$ são dadas o custo $W_{k}$. Uma similaridade $s(X, Y)$ é dada entre os elementos das strings $X$ e $Y$. Para encontrar o par de segmentos com maior similaridade, é construída a matriz $H$ pela seguinte recorrência:

$$
\begin{gathered}
H(k, 0)=H(0, l) \text { para } 0 \leq k \leq|X| \text { e } 0 \leq l \leq|Y| \\
H(i j)=\max \left\{\begin{array}{c}
H(i-1, j-1)+s\left(X_{i}, Y_{j}\right) \\
H(i-k, j)-W_{k} \\
H(i, j-l)-W_{l} \\
0
\end{array}\right\}, 1 \leq i \leq|X| \text { e } 1 \leq j \leq|Y|
\end{gathered}
$$

A fórmula para $H_{i j}$ considera a possibilidade de terminar os segmentos em qualquer $X_{i}$ ou $Y_{j}$. Assim, se $X_{i}$ e $Y_{j}$ estão relacionados, a similaridade é $H(i-1, j-1)+$ $s\left(X_{i}, Y_{j}\right)$. Se $X_{i}$ está ao final de uma exclusão de comprimento $k$, a similaridade é $H(i-k, j)-W_{k}$. Se $Y_{j}$ está ao final de uma exclusão de comprimento $l$, a similaridade é $H(i, j-l)-W_{l}$. Finalmente, um zero é incluído para prevenir os cálculos negativos da similaridade, indicando que não há semelhança até $X_{i}$ e $Y_{j}$.

As seguintes métricas são exemplos de métricas que calculam a similaridade entre duas strings: 
- O Coeficiente de Dice retorna valores entre zero e 1, sendo 1 quando as sequências de entrada são completamente iguais. Esta medida pode ser utilizada também no modelo vetorial como na comparação direta de strings. A medida de similaridade entre duas strings $(X, Y)$ é definida como o dobro do número de tokens comuns de $X$ e $Y$, dividido pelo número total de tokens testados em ambas entidades. A função é definida pela seguinte equação:

$$
\operatorname{sim}_{\text {Dice }}(X, Y)=\frac{2 * Q(X \cap Y)}{Q(X)+Q(Y)}
$$

onde a função $Q(\cdot)$ retorna a quantidade de tokens que compõem uma string. Por exemplo, para calcular a similaridade entre duas strings: $X=$ "Universidade de São Paulo" e $Y=$ "Universidade Federal de São Carlos". Contabilizamos a quantidade de tokens que contém $X, Y$ e o número de tokens em comum. Assim, a distância dist $_{\text {Dice }}$ entre $X$ e $Y$ será:

$$
\operatorname{sim}_{\text {Dice }}(X, Y)=\frac{2 * 3}{4+5}=0,667
$$

- A similaridade do Cosseno é uma medida semelhante ao Coeficiente de Dice. Para medir a similaridade entre duas strings, utiliza-se a Equação 3.18:

$$
\operatorname{sim}_{\text {cosseno_S }}(X, Y)=\frac{Q(X \cap Y)}{\sqrt{Q(X) * Q(Y)}}
$$

Utilizando o exemplo anterior, neste caso a similaridade entre $X$ e $Y$ é 0,671 .

- Outra métrica utilizada para medir a similaridade entre strings é o Coeficiente de Matching. A métrica retorna valores entre zero e 1, sendo que 1 indica que as strings de entrada são completamente iguais. A função é definida pela seguinte equação:

$$
\operatorname{sim}_{\text {matching }}(X, Y)=\frac{Q(X \cap Y)}{\max \{Q(X), Q(Y)\}}
$$

onde max retorna a quantidade de tokens da string com mais tokens. Para o exemplo anterior, a similaridade sim $_{\text {matching }}$ entre as strings $X$ e $Y$ é 0,6 .

- O Coeficiente de Overlap é uma medida pela qual, se uma string $X$ é uma subsequência de $Y$, ou o inverso, então, o coeficiente de similaridade será 1 . A métrica é definida pela Equação 3.20:

$$
\operatorname{sim}_{\text {overlap }}(X, Y)=\frac{P(X \cap Y)}{\min \{P(X), P(Y)\}}
$$


onde a função $P(\cdot)$ retorna a quantidade de caracteres que compõem uma string. Seguindo com o exemplo anterior, o valor de dist ${ }_{\text {overlap }}$ para $X$ e $Y$ :

$$
\operatorname{sim}_{\text {overlap }}(X, Y)=\frac{19}{\min \{25,34\}}=0,76
$$

- Q-gram é outra medida de similaridade entre strings. Uma string é dividida em substrings de comprimento variável $q(q \geq 1)$, por meio do "deslizamento" de uma janela de comprimento $q$ sobre a string de entrada. Por exemplo, a string $X=$ "Universidade de São Paulo" seria dividida nas seguintes substrings para $q=3$ : (\#,\#,U); (\#,U,n); (U,n,i); (n,i,v); (i,v,e); (v,e,r); (e,r,s); (r,s,i); (s,i,d); (i,d,a); (d,a,d); (a,d,e); (d,e, ); (e, ,d); ( ,d,e); (d,e, ); (e, ,S); ( ,S,ã); (S,ã ,o); (ã ,o, ); (o, ,P); $(, \mathrm{P}, \mathrm{a}) ;(\mathrm{P}, \mathrm{a}, \mathrm{u}) ;(\mathrm{a}, \mathrm{u}, \mathrm{l}) ;(\mathrm{u}, \mathrm{l}, \mathrm{o}) ;(\mathrm{l}, \mathrm{o}, \%)$ e $(\mathrm{o}, \%, \%)$, com \# e \% indicando o inicio e o final da string. Matemáticamente é definido como:

$$
\operatorname{sim}_{\text {qgram }}(X, Y)=\frac{2 * G(X \cap Y)}{G(X)+G(Y)}
$$

onde a função $G(\cdot)$ retorna o número de substrings de comprimento $q$ na string de entrada.

Outra abordagem é o cálculo da dissimilaridade entre documentos baseado em uma aproximação da complexidade de Kolmogorov (Li et al., 2004). Esta abordagem busca quantificar a complexidade (quantidade de informação) de strings e outros objetos de forma objetiva e absoluta. No entanto, a complexidade de Kolmogorov não é uma função computável. Apesar disso, uma aproximação para a verdadeira complexidade é possível usando algoritmos de compressão. Seguindo este abordagem, Cilibrasi e Vitanyi (Cilibrasi e Vitanyi, 2005) propuseram uma medida denominada Normalized Compression Distance, em que dadas duas strings $X$ e $Y$ calcula a seguinte aproximação para a complexidade de Kolmogorov:

$$
\operatorname{dist}_{N C D}(X, Y)=\frac{C(X Y)-\min \{C(X), C(Y)\}}{\max \{C(X), C(Y)\}}
$$

onde $X Y$ é a concatenação de $X$ e $Y$, e $C(X)$ é o tamanho em bytes da compressão da string $X$ por um compressor. O valor de $\operatorname{dist}_{N C D}(X, Y)$ é um número entre zero e $1+\delta$, onde $\delta$ é uma função de limitação do compressor. Quanto menor o valor de $\operatorname{dist}_{N C D}(X, Y)$, mais similares são $X$ e $Y$. Algoritmos como o LZW (Lempel-Ziv-Welch) e PPMZ (Predição por coincidência parcial com string de tamanho ilimitado) podem ser utilizados como compressores. 
A scaled Normalized Compression Distance (Telles et al., 2007) é uma métrica baseada na distância $N C D$. Esta retorna zero quando aplicada sobre dois documentos idênticos. A métrica visa superar os problemas relacionados à precisão do compressor e é dada pela Equação 3.24:

$$
\operatorname{dist}_{N C D s}(X, Y)=\operatorname{dist}_{N C D}(X, Y)+\frac{\operatorname{dist}_{N C D}(X, X)+\operatorname{dist}_{N C D}(Y, Y)}{2}
$$

A grande vantagem de métricas sobre strings é não requerer representações intermediárias da coleção de documentos, como o modelo do espaço vetorial. Em coleções que sofrem adição ou remoção de novos documentos, a representação vetorial deve ser gerada novamente a cada alteração da coleção. Medidas de similaridade/dissimilaridade entre strings não sofrem com este problema, pois nenhuma representação intermediária adicional é utilizada. Dessa forma a adição ou remoção de documentos em uma coleção não altera as relações de similaridade entre os documentos que já estavam presentes. No entanto, o cálculo destas distâncias tem, em geral, um alto custo computacional, tornando o processo de computar as dissimilaridades lento.

\subsection{Considerações Finais}

Neste capítulo foram apresentados dois modelos vetoriais como técnicas de representação de documentos: VSM e iVSM. Apesar do VSM ser útil na geração de mapas visuais de conjuntos de textos, não é incremental, ou seja, novas adições à coleção forçam o recálculo de todo o espaço e das dissimilaridades entre instâncias. Nesse sentido, o iVSM, baseado no VSM, foi proposto por Pinho (2009). Ambos os modelos descrevem um documento como um vetor de frequências de termos. Em ambos os casos, para melhorar a qualidade da representação gerada e reduzir sua dimensionalidade são utilizados métodos como a remoção de stopwords, lematização e cortes de Luhn.

Também foram apresentadas algumas métricas para computar a similaridade ou dissimilaridade entre documentos. Uma primeira possibilidade são as métricas aplicáveis aos modelos vetoriais, como a distância Euclidiana, de Manhattan e do Cosseno, esta última empregada para medir distância em conjuntos de dados esparsos. Uma segunda possibilidade são as métricas que calculam a similaridade entre dois documentos de forma direta, aplicando operações simples sobre o tamanho compacto dos documentos (strings), retor- 
nando o menor número de operações de edição necessárias para transformar uma string em outra. 


\subsection{Considerações Iniciais}

Uma vez definido o cálculo de dissimilaridade para um conjunto de textos, a etapa da geração de um mapa visual tem como objetivo exibir esses textos em um espaço bi ou tridimensional. Técnicas de agrupamento também podem ser aplicadas para identificar grupos de textos semelhantes, desenhar fronteiras ou associar legendas aos grupos.

Neste capítulo são apresentadas algumas das técnicas disponíveis para a geração de layouts de conjuntos textuais mediante projeções multidimensionais e árvores filogenéticas. Para finalizar, algumas medidas de avaliação da qualidade de mapas visuais são apresentadas.

\subsection{Técnicas de Projeção Multidimensional}

Uma das abordagens de visualização utilizadas neste projeto foram projeções multidimensionais. O objetivo de tais técnicas é criar representações visuais, em baixa dimensionalidade, que permitam descrever visualmente e eficientemente a distribuição de dados multidimensionais volumosos. Em geral, uma técnica de projeção mapeia dados $n$-dimensionais em um espaço $p$-dimensional para $p \leq n$, em geral, com $p=1,2$ ou 3 para espaços visuais, 
de forma que, no espaço projetado, as distâncias entre os elementos gráficos correspondam ao grau de dissimilaridade dos dados no espaço original. A seguir, uma definição formal de projeção multidimensional, segundo Tejada et al. (2003) para um espaço bidimensional $(p=2)$ :

Definição 4.1 (Projeção Multidimensional) : Dado um conjunto de dados $X$ no espaço $n$-dimensional e um critério de dissimilaridade dist $: \mathbb{R}^{n} \rightarrow \mathbb{R}$ entre instâncias $n$-dimensionais dos dados. Pretende-se encontrar um conjunto de pontos $Y$ em $\mathbb{R}^{2}$ tal que se $\alpha: X \rightarrow Y$ é uma relação bijetora e dist $t_{2}: \mathbb{R}^{2} \rightarrow \mathbb{R}$ um critério de proximidade em $\mathbb{R}^{2}$, visando tornar a diferença $\mid \operatorname{dist}\left(x_{i}, x_{j}\right)-$ dist $_{2}\left(\alpha\left(x_{i}\right), \alpha\left(x_{j}\right)\right) \mid$ tão próxima de zero quanto possivel, $\forall x_{i}, x_{j} \in X$.

Cada ponto do conjunto $Y$ representa uma instância dos dados cujas coordenadas podem ser usadas para gerar uma representação visual. A posição desses pontos na representação reflete informações sobre a estrutura do conjunto, expressa por meio da vizinhança espacial entre pares de instâncias na projeção, formação de grupos visuais e o relacionamento entre tais grupos. Assim, elementos posicionados próximos entre si indicam que as instâncias de dados que eles representam são similares, com relação à medida de dissimilaridade considerada.

A Definição 4.1 requer uma medida de dissimilaridade entre elementos $n$-dimensionais. Assim, a matriz de dissimilaridade é a base para a projeção. Dado um conjunto de $N$ documentos, a matriz de dissimilaridade contém as distâncias entre pares de documentos. A Tabela 4.1 representa uma matriz de distâncias, sendo que $\operatorname{dist}\left(d_{i}, d_{j}\right)$ representa a distância entres os documentos $i$ e $j$ segundo alguma das métricas de dissimilaridade, por exemplo as apresentadas na Seção 3.3.

\begin{tabular}{|ccccc|}
\hline 0 & & & & \\
$\operatorname{dist}\left(d_{2}, d_{1}\right)$ & 0 & & & \\
$\operatorname{dist}\left(d_{3}, d_{1}\right)$ & $\operatorname{dist}\left(d_{3}, d_{2}\right)$ & 0 & & \\
$\vdots$ & $\vdots$ & $\vdots$ & $\ddots$ & \\
$\operatorname{dist}\left(d_{N}, d_{1}\right)$ & $\operatorname{dist}\left(d_{N}, d_{2}\right)$ & $\operatorname{dist}\left(d_{N}, d_{3}\right)$ & $\cdots$ & 0 \\
\hline
\end{tabular}

Tabela 4.1: Matriz de distâncias.

No grupo de pesquisa Visualization, Imaging and Computer Graphics (VICG), diversas técnicas de projeção multidimensional vêm sendo investigadas e incorporadas à plataforma de visualização VisPipeline. A plataforma, desenvolvida em Java, implementa várias técnicas de visualização multidimensional e de posicionamento de pontos baseadas 
em similaridade de conteúdo dos objetos de dados, bem como funcionalidades de interação, pré-processamento e algumas ferramentas de análise. A seguir apresentamos algumas das técnicas para a geração de mapas visuais disponíveis na ferramenta VisPipeline e que aceitam como entrada uma matriz de dissimilaridade. Para ilustrar essas técnicas foi utilizado o corpus de 574 artigos científicos classificados em três áreas de pesquisa: Case-Based Reasoning- representado em vermelho nas figuras a seguir, Inductive Logic Programming em verde e Information Retrieval em azul (mais detalhes na Seção 5.3).

\subsubsection{Interactive Document Map}

A técnica IDMAP foi desenvolvida com o objetivo de projetar e analisar coleções de documentos (Minghim et al., 2006). É baseada na combinação de duas técnicas: (i) FastMap (Faloutsos e Lin, 1995), para criar uma projeção inicial das instâncias; e (ii) ForceScheme (Tejada et al., 2003) utilizada para ajustar os pontos projetados de modo a compensar a informação perdida na projeção inicial. A combinação das duas técnicas tem como fim reduzir o custo computacional da ForceScheme mediante a geração de um layout inicial com a técnica FastMap. Embora a técnica gere representações que conseguem refletir as relações de similaridade entre as instâncias, ela apresenta limitações de tempo quando aplicada a grandes coleções de textos. O complexidade da técnica é $O\left(N^{2}\right)$, onde $N=|X|$.

A Figura 4.1 exibe um mapa de documentos gerado com a IDMAP, a partir de uma matriz de distâncias calculadas sobre um modelo vetorial da coleção e utilizando a distância do cosseno.

\subsubsection{Projection by Clustering}

A técnica ProjClus busca manter ou melhorar a qualidade dos layouts gerados com a técnica IDMAP, mas reduzindo seu custo computacional (Paulovich e Minghim, 2006). A geração de um mapa visual sumariza-se em três passos: (i) primeiro, os $N$ elementos de um conjunto de dados são divididos em $\sqrt{N}$ agrupamentos empregando o algoritmo bisecting k-means (Tan et al., 2005). (ii) Em seguida, os centroides de cada agrupamento são calculados e projetados utilizando FastMap e ForceScheme. Posteriormente, cada conjunto de pontos pertencente a cada agrupamento é projetado separadamente, em um espaço normalizado. (ii) Por último, os agrupamentos já projetados são posicionados no desenho final do mapa de acordo com a posição de seus centroides. A complexidade do algoritmo 


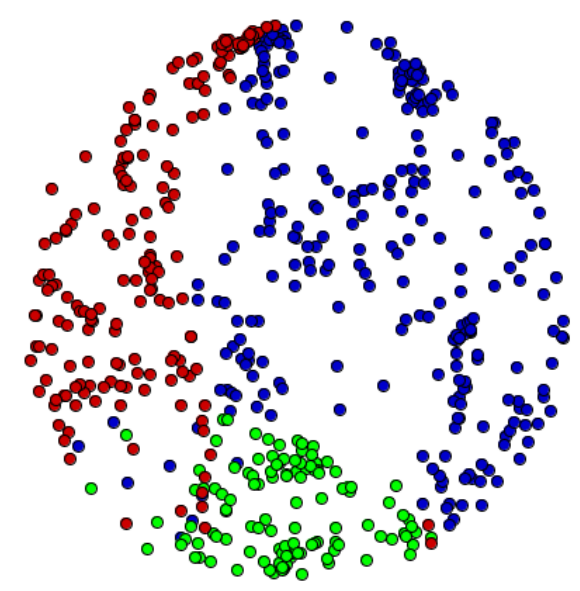

Figura 4.1: Mapa do conjunto de documentos CBR-ILP-IR gerado com a técnica IDMAP.

é $O\left(N^{\frac{3}{2}}\right)$. A Figura 4.2 exibe um mapa de documentos gerado com a técnica ProjClus, a partir do mesmo conjunto de dados e representação empregada no caso anterior.

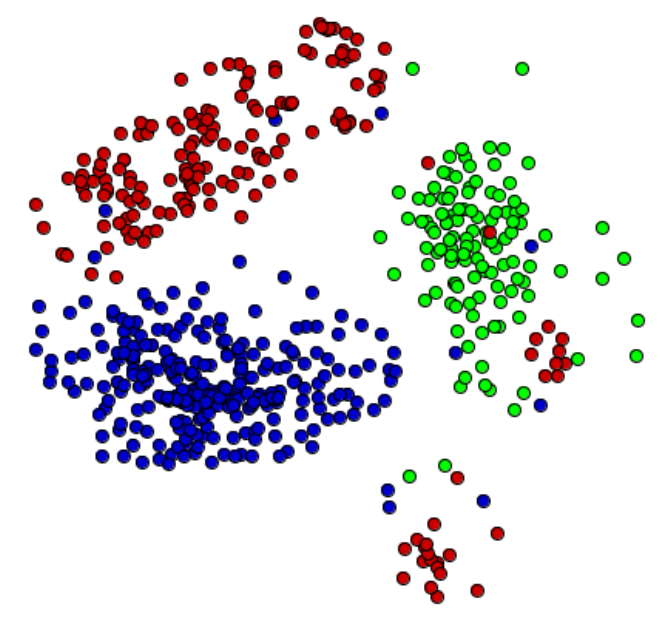

Figura 4.2: Mapa gerado para o conjunto CBR-ILP-IR usando ProjClus.

\subsubsection{Least Square Projection}

A técnica de projeção multidimensional LSP (Paulovich, 2008; Paulovich et al., 2008) é capaz de projetar conjuntos de dados em um tempo computacional satisfatório, preservando as relações de vizinhança entre as instâncias $n$-dimensionais no espaço projetado. Primeiramente, um sub-conjunto de pontos $C$, chamado de pontos de controle, é selecionado 
do conjunto original e projetado em $\mathbb{R}^{p}$ por algum método de redução de dimensionalidade, por exemplo FastMap. Logo, considerando-se as relações de similaridade entre os objetos do espaço original e as coordenadas cartesianas dos pontos de controle $C$, constrói-se um sistema linear cuja solução define as posições dos pontos restantes em $\mathbb{R}^{p}$, cuja complexidade é $O(N \sqrt{k})$, onde o índice $k$ representa o número de condição da matriz $A^{T} A$ (Shewchuk, 1994). Portanto, a complexidade final da LSP é $O(\max N \sqrt{N}, N \sqrt{k})$. A Figura 4.3 exibe um mapa gerado com a técnica LSP para o mesmo conjunto de dados e representação utilizados anteriormente.

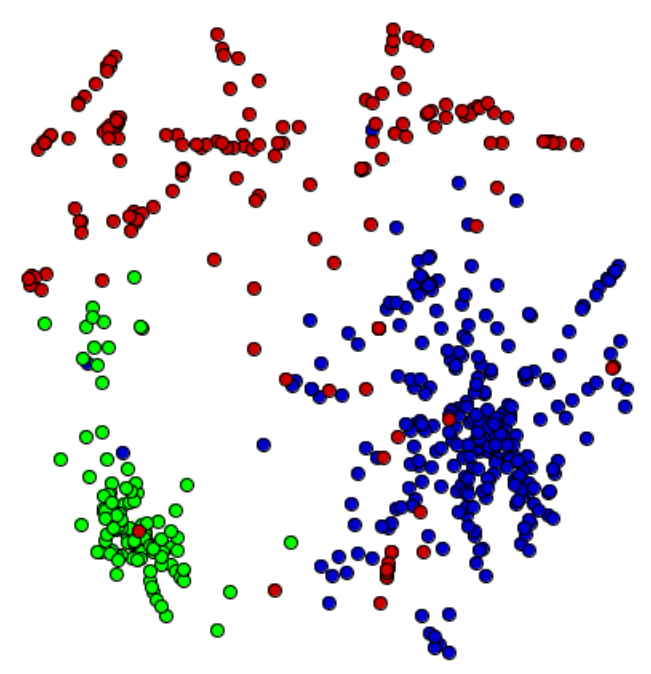

Figura 4.3: Mapa de documentos do CBR-ILP-IR gerado com a técnica LSP.

\section{3 Árvores Filogenéticas}

A filogenia é o termo comumente utilizado para denotar o estudo do processo de evolução de grupos de espécies, assim como das relações existentes entre elas (Setubal e Meidanis, 1997). Essas relações de evolução podem ser ilustradas com a construção de árvores filogenéticas, cujas folhas representam os indivíduos ou espécies, os nós internos representam ancestrais e o tamanho dos caminhos ou ramos representam a distância evolutiva. A Figura 4.4 ilustra um exemplo de árvore filogenética construída com a ferramenta MATLAB. A imagem descreve as diferentes evoluções de cinco diferentes espécies de hominídeos, usando os códigos de adesão para as sequencias mitocondriais D-loop ${ }^{1}$. Dela podemos

\footnotetext{
${ }^{1}$ Displacement loop ou D-loop é um termo utilizado em biologia molecular para definir um circuito de ssDNA que é formado, por exemplo, quando um fragmento de ssDNA curto hibridiza com uma sequência complementar de um dos fios de dsDNA (Singleton, 2010)
} 
inferir que "German_Neanderthal" e "Russian_Neanderthal" estão mais próximos entre si do que das outras espécies. A informação foi obtida do National Center for Biotechnology Information (NCBI) em http://www.ncbi.nlm.nih.gov/.

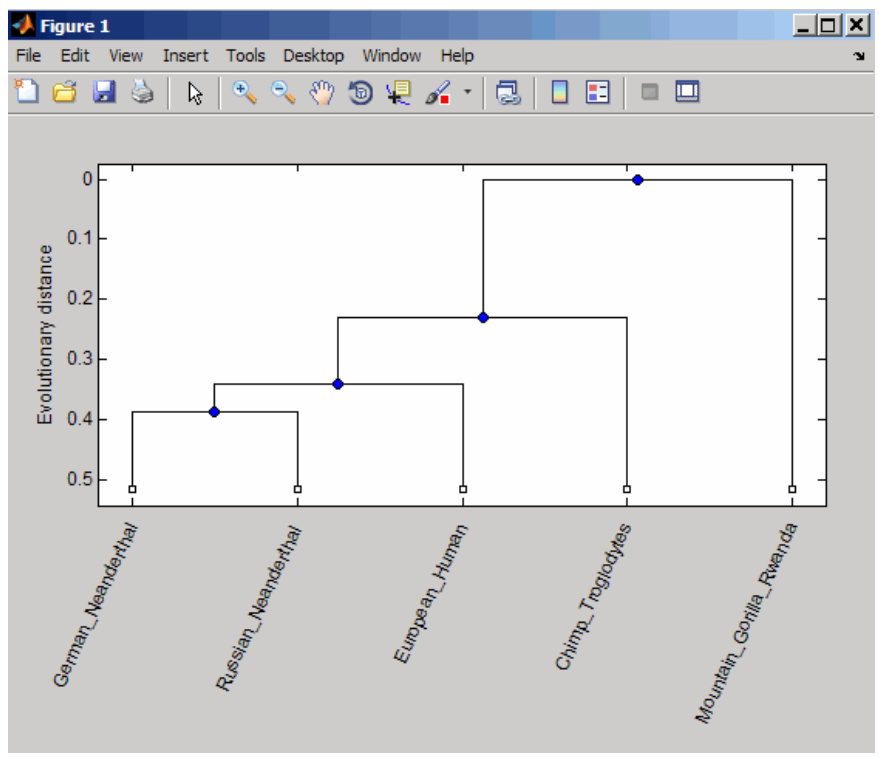

Figura 4.4: Árvore filogenética gerada com a ferramenta MATLAB

As árvores filogenéticas podem ser utilizadas como árvores de similaridade, sendo que, as folhas representam as instâncias de um conjunto, os nós internos representam objetos hipotéticos e o comprimento dos arcos define a similaridade entre as instâncias.

Um dos métodos mais representativos para a construção de árvores filogenéticas é o algoritmo NJ, apresentado a seguir.

\subsubsection{Neighbor Joining}

Cuadros et al. (2007) estudaram a aplicação de árvores filogenéticas usando a técnica NJ baseada no layout radial, para visualizar coleções de documentos textuais. O método NJ, proposto por Saitou e Nei (1987), constrói uma árvore sem raiz, com topologia criada a partir de uma matriz de distâncias, segundo algum critério de reconstrução. O critério de reconstrução busca encontrar os dois objetos com menor valor no cálculo da soma das distância entre ramos (chamados de vizinhos). Para isso aplica-se a Equação 4.1:

$$
S_{i j}=\frac{1}{2(N-2)}\left[\sum_{r \neq i, j}^{N}\left(\text { dist }_{i r}+d_{j r}\right)+\frac{1}{2} \text { dist }_{i j}+\frac{1}{N-2} \sum_{(r, l \neq i, j) \&(r<l)}^{N} \operatorname{dist}_{r l}\right]
$$


onde $d_{i s t} t_{i j}$ é o valor na matriz de distância relativo aos elementos dos índices $i, j$ e os índices $r$ e $l$ representam todos os objetos exceto os selecionados, ou seja, $i$ e $j$. Escolhido o par de objetos vizinhos a serem agrupados em um novo nó $A$, o tamanho dos novos ramos é calculado pelo método Fitch-Margoliash (Fitch e Margoliash, 1967):

$$
\begin{aligned}
L_{i A} & =\frac{d i s t_{i j}+d i s t_{i z}-d i s t_{j z}}{2} \\
L_{j A} & =\frac{d i s t_{i j}+d i s t_{j z}-d i s t_{i z}}{2}
\end{aligned}
$$

onde $z$ representa o grupo dos objetos da árvores, com exceção dos objetos $i$ e $j$. Os valores dist $_{i z}$ e dist ${ }_{j z}$ são determinados pelas distâncias de $i$ e $j$ aos demais objetos:

$$
\begin{aligned}
d i s t_{i z} & =\frac{\sum_{r \neq j}^{N} d i s t_{i r}}{N-2} \\
d i s t_{j z} & =\frac{\sum_{r \neq i}^{N} d i s t_{i r}}{N-2}
\end{aligned}
$$

Logo, a distância entre o novo objeto e os demais é calculada, $\forall r \leq N$ excluindo $i$ e $j$ :

$$
\operatorname{dist}_{i-j, z}=\frac{d i s t_{i r}+d i s t_{j r}}{2}
$$

Uma das vantagens das árvores NJ é que oferecem uma visão tanto global como local da estrutura do conjunto de dados visualizado. As principais limitação desta técnica são a complexidade computacional, de ordem $O\left(N^{3}\right)$ e a saturação do espaço visual devido à presença dos nós hipotéticos. Assim, na literatura existem diferentes trabalhos com o objetivo de melhorar a velocidade do algoritmo NJ, como o de Mailund et al. (2006), que tentam diminuir o tempo de busca para encontrar o par de vizinhos mais próximos usando a estrutura Quad-tree. No melhor dos casos a ordem de processamento fica em $O\left(N^{2}\right)$. Outro trabalho foi apresentado por Paiva et al. (2011), no qual o tempo de processamento é reduzido implementando os algoritmos Fast e Rapid Neighbor Joining, além de aliviar a sobrecarga visual utilizando um procedimento de rescrita linear do grafo baseado na promoção de nós. A Figura 4.7 apresenta um mapa visual gerado com a técnica NJ utilizando o algoritmo Rapid Neighbor Joining, a partir da mesma representação e medida de distância utilizados anteriormente. 


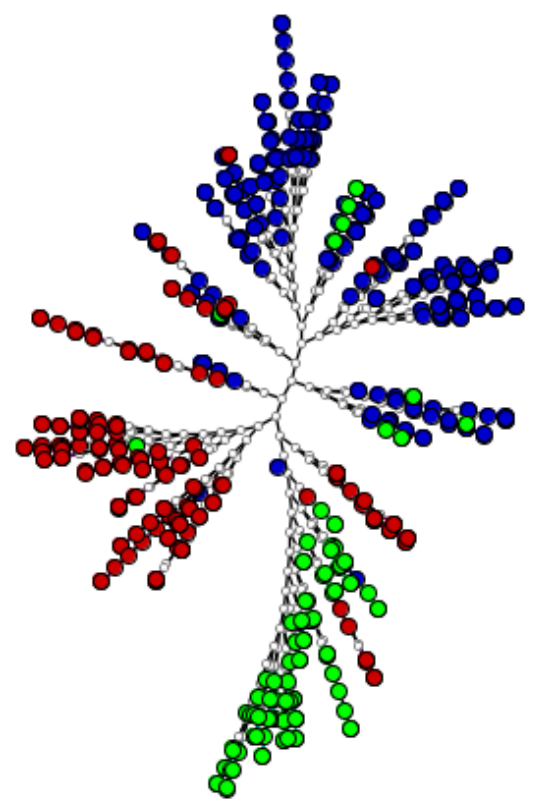

Figura 4.5: Mapa de documentos do conjunto CBR-ILP-IR gerado com a técnica NJ

\subsection{Medidas de Avaliação da Qualidade de Mapas Visuais}

Existem algumas medidas objetivas para comparar a qualidade visual de diferentes mapas gerados por projeções e árvores utilizando diferentes medidas de distância e modelos de representação. Um exemplo é Informação Mútua Normalizada (NMI) (Srivastava e Sahami, 2009) que mede a precisão de um algoritmo de agrupamento, por exemplo $k$-means, ou de uma projeção multidimensional para refletir, no espaço projetado, as classes existentes no conjunto de dados. Outro exemplo é a Neighborhood Preservation (NP) que visa avaliar a preservação da vizinhança dos objetos multidimensionais no layout final, sendo que a precisão final é a média das precisões para cada ponto Paulovich (2008). Para nossos estudos consideramos duas: o Coeficiente de Silhueta (CS), que tenta capturar a qualidade dos agrupamentos identificáveis no espaço de alta dimensionalidade (ou no espaço projetado), e a curva Neighborhood Hit (NH), que tenta captar em que medida um layout preserva a separação das classes conhecidas.

\subsubsection{Coeficiente de Silhueta}

O Coeficiente de Silhueta (CS) ou índice da silhueta, avalia a qualidade de um agrupamento por meio das diferenças entre as distâncias intra grupo e inter grupo entre as instâncias de dados (Kaufman e Rousseeuw, 2005). Para conjuntos de dados classificados, 
um grupo pode ser definido pelas instâncias pertencentes a uma mesma classe. O processo para calcular o valor de silhueta para uma instância individual é composto por três passos:

1. Para a $i$-ésima instância, calculamos a média das distâncias entre $i$ e todas as outras instâncias pertencentes ao mesmo grupo. Esse valor será denominado $a_{i}$ e dá uma medida da coesão do grupo.

2. Para a $i$-ésima instância, calculamos a média das distâncias entre $i$ e todas as outras instâncias de outros grupos. O valor mínimo encontrado será denominado $b_{i}$ e dá uma medida da separação entre os grupos.

3. Para a $i$-ésima instância, a silhueta é

$$
s_{i}=\frac{\left(b_{i}-a_{i}\right)}{\max \left(a_{i}, b_{i}\right)}
$$

Logo, o CS de um agrupamento é calculado pela média da silhueta de todas as $N$ instâncias do conjunto de dados:

$$
C S=\frac{1}{N} \sum_{i=1}^{N} \frac{\left(b_{i}-a_{i}\right)}{\max \left(a_{i}, b_{i}\right)}
$$

O valor do índice da silhueta está no intervalo $[-1,1]$. Se o agrupamento possui apenas um elemento, assume-se que o valor da silhueta é zero. Valores maiores do CS indicam melhor coesão e separação entre os grupos. Aplicado no espaço multidimensional, o coeficiente de silhueta não avalia o layout, mas a capacidade do algoritmo de distância de identificar grupos coesos e bem separados. A Figura 4.6 apresenta os valores do coeficiente de silhueta para os quatro mapas visuais apresentados anteriormente.

\subsubsection{Neighborhood Hit}

A Neighborhood Hit (NH) (Paulovich et al., 2008) é uma estratégia para avaliar numericamente quão destacadas estão as classes pré-existentes nos mapas visuais gerados com árvores NJ e projeções multidimensionais. Dada uma pré-classificação, a estratégia visa analisar a capacidade do layout de preservar as classes em uma mesma vizinhança, favorecendo a sua percepção. A ideia é tomar os $k$-vizinhos mais próximos de um ponto projetado no plano e verificar que proporção desses pertencem à mesma classe do ponto de referência. O cálculo desses vizinhos é diferente para cada técnica, árvores ou projeções. No caso das projeções, o cálculo é realizado em função de uma distância, por exemplo a distância Euclidiana, entre os pontos no plano de projeção segundo uma ordem crescente (Paulovich et al., 2006). Quando mais separados e agrupados estiverem os pontos 


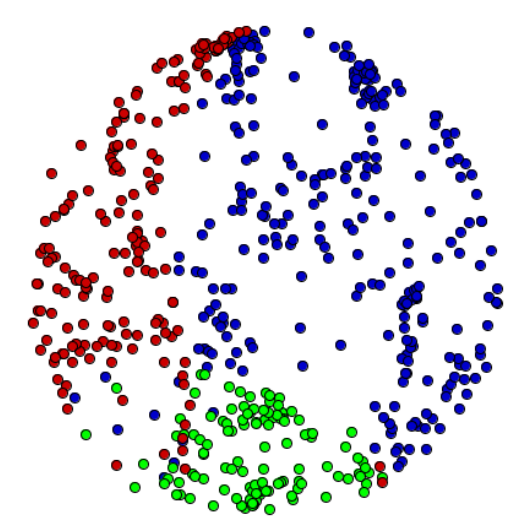

(a) IDMAP: $\mathrm{CS}=0.264$

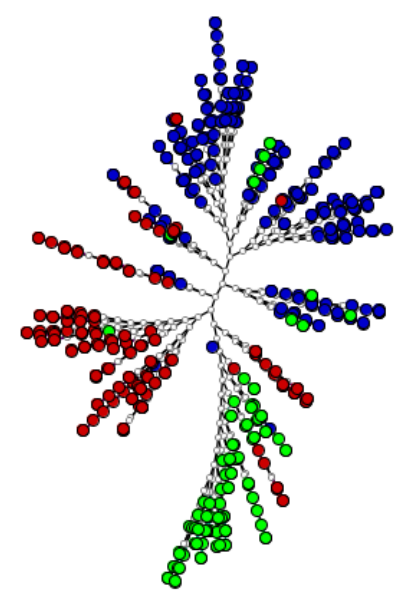

(c) NJ: $\mathrm{CS}=0.221$

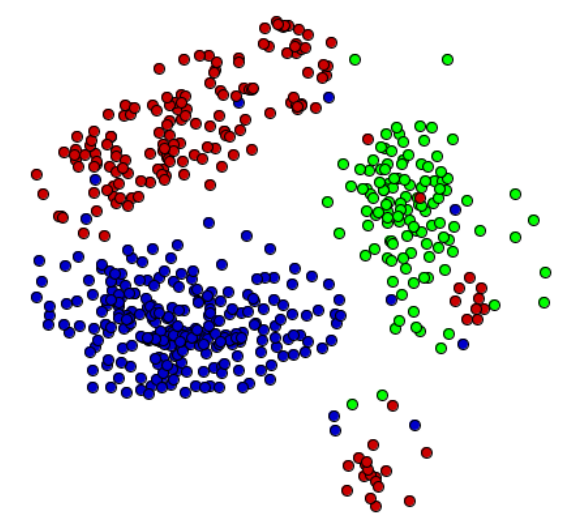

(b) ProjClus: $\mathrm{CS}=0.423$

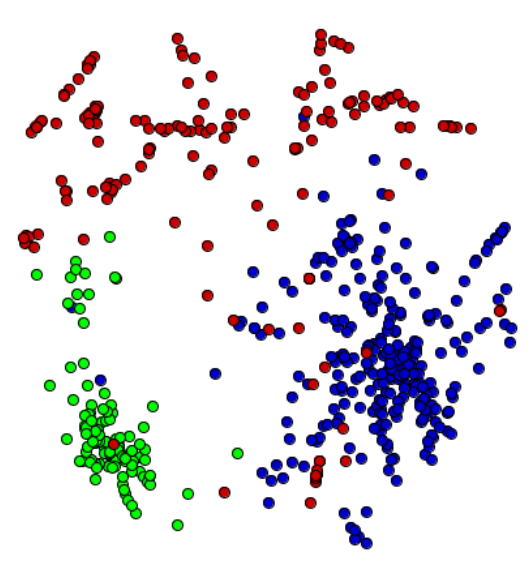

(d) LSP: $\mathrm{CS}=0.52$

Figura 4.6: Quatro diferentes projeções com seus respectivos coeficientes de silhueta para o conjunto de artigos científicos.

no layout final, de acordo com as classes, maior será a precisão. Para as árvores NJ, a precisão é determinada em duas fases: (i) percorrendo a árvore segundo o critério de vizinhança para NJ e (ii) calculando quantos desses vizinhos pertencem à mesma classe. A travessia determinada pela árvore respeita a ordem em que um par de nós folha for unido a um nó interno. Assim, para obter os $k$ vizinhos mais próximos a um nó $u$ são realizados os seguintes passos: (i) visitar o nó $A$ ancestral imediato de $u$. (ii) Logo, percorrer todos os nós oriundos do primeiro vizinho mais próximo de $A$. (iii) Da mesma forma, percorrer todos os nós oriundos do segundo vizinho mais próximo de $A$. (iv) Finalmente, percorrer todos os nós antepassados do terceiro vizinho mais próximo de $A$. A árvore é percorrida 
até obter a sequencia $\left(u_{1}, u_{2}, \ldots, u_{k}\right)$ para as $k$ folhas vizinhas de $u$. Esse processo é realizado para todos os nós folhas da árvore.

No trabalho de Cuadros (2007) são comparados os resultados da precisão obtidos de mapas gerados com árvores NJ e projeções multidimensionais para diferentes conjuntos de documentos textuais. Os autores concluíram que, para amostras variando de um a trinta vizinhos mais próximos, a técnica NJ apresentou a maior porcentagem de separação das classes existentes nos conjuntos de dados testados. Os experimentos também comprovaram que a precisão visual dos pontos no layout é aplicável às distâncias computadas sobre a representação vetorial dos textos, como também a distâncias de cálculo direto, i.e., obtidas comparando diretamente as strings. Isso pode ser visto na Figura 4.7, que apresenta as curvas NH de diferentes técnicas de posicionamento de pontos obtidas de matrizes de distância calculadas usando a medida NCDs para o conjunto de artigos científicos CBR-ILP-IR-SON (documentos do conjunto CBR-ILP-IR mais artigos científicos na área de Sonificação (SON-Sonification)). Observamos que a curva de precisão gerada para a árvore NJ apresentou os melhores resultados (valores mais próximos a 1), seguido pelas técnicas LSP e ProjClus.

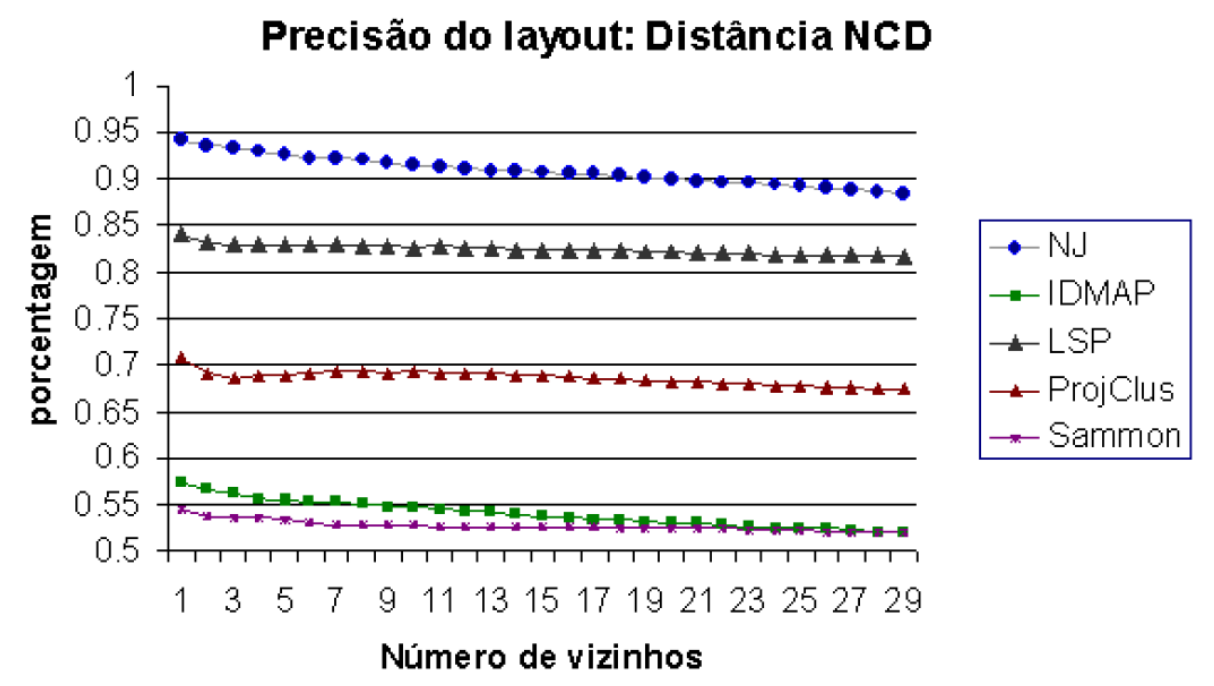

Figura 4.7: Curvas de precisão de diferentes técnicas para a geração de mapas visuais usando matrizes de distância calculadas com NCD. Extraído de (Cuadros, 2007)

\subsection{Considerações Finais}

Neste capítulo foram apresentadas técnicas de projeção multidimensional e árvores filogenéticas para gerar representações visuais, em baixa dimensionalidade, de conjuntos de 
dados multidimensionais, como textos. Adicionalmente, diferentes medidas de avaliação da qualidade visual de diferentes mapas visuais gerados por técnicas como NJ e LSP, foram apresentadas.

Com este capítulo terminamos a revisão bibliográfica dos conceitos que serão aplicados em nossos estudos. Assim, no próximo capítulo são apresentados os elementos e as configurações utilizados no estudo comparativo. 


\section{Descrição do Estudo Experimental}

\subsection{Considerações Iniciais}

Este trabalho de mestrado teve por objetivo estudar como o cálculo da similaridade adotado para gerar a matriz de distância afeta o layout final gerado por projeções multidimensionais e árvores filogenéticas, como diferentes medidas se comparam neste contexto, bem como investigar a viabilidade de soluções incrementais. O cálculo da similaridade é realizado de duas maneiras: aplicando medidas de distância sobre uma representação vetorial dos documentos, ou comparando diretamente strings. Basicamente, as matrizes geradas são visualizadas em uma representação gráfica que revela as relações de similaridade entre os textos analisados.

O diagrama da Figura 5.1 sumariza o processo geral de construção de mapas visuais adotado neste trabalho. Inicialmente, dado um conjunto de textos, a matriz de dissimilaridade é computada. Para isso, duas abordagens são consideradas:

- A primeira aplica alguma métrica para estabelecer o critério de dissimilaridade sobre uma representação vetorial dos documentos (VSM ou iVSM, descritas no Capítulo 3).

- A segunda calcula diretamente a dissimilaridade utilizando alguma métrica para comparar strings textuais. 
Obtida esta matriz de dissimilaridade, uma representação visual é gerada utilizando técnicas de projeção multidimensional ou árvores filogenéticas. Finalmente, os layouts são avaliados visual e quantitativamente apoiados por técnicas como NH e CS, descritas na Seção 4.4 .

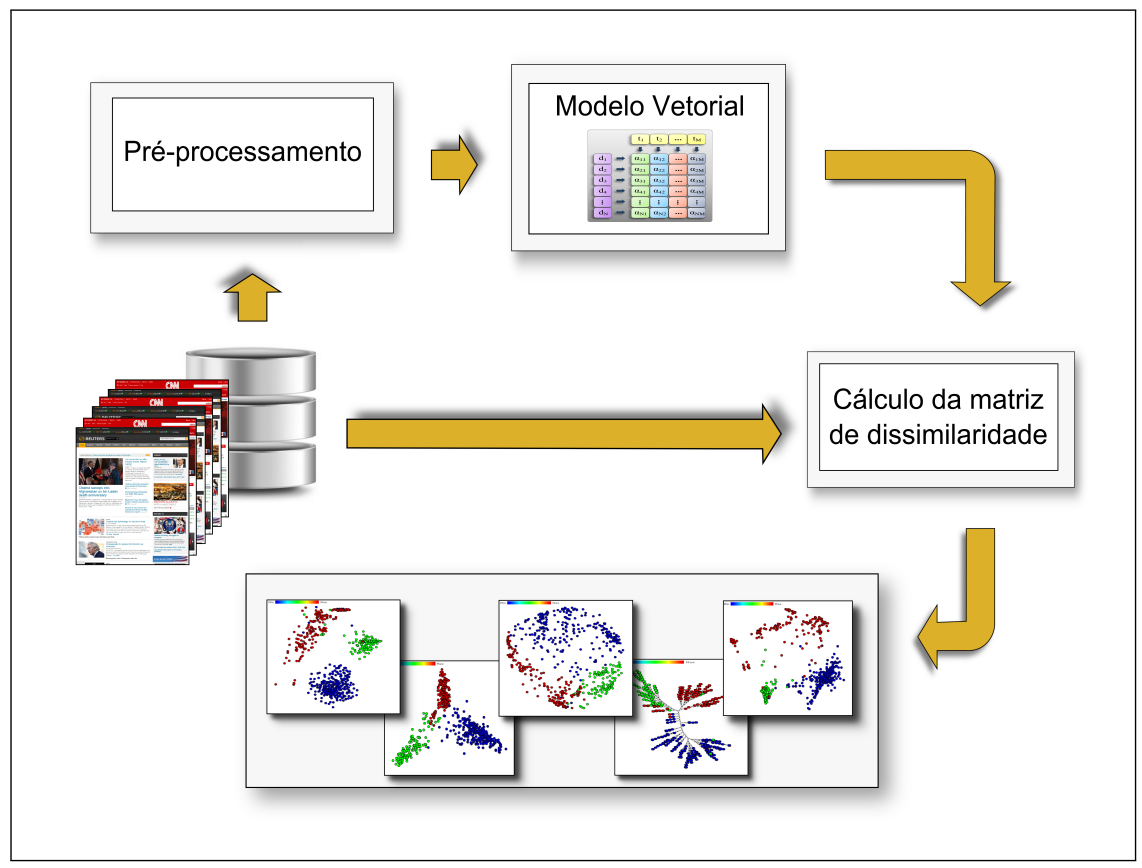

Figura 5.1: Pipeline de visualização

As duas abordagens descritas para o cálculo da matriz de dissimilaridade foram incorporadas à plataforma de visualização VisPipeline. Esta plataforma permite-nos conectar visualmente componentes e ferramentas para criar pipelines de visualização e gerar mapas visuais.

Este capítulo está organizado como segue: na Seção 5.2 apresentam-se os módulos incorporados à ferramenta VisPipeline, desenvolvidos para viabilizar o nosso estudo comparativo. Em seguida, na Seção 5.3 três conjuntos de textos de natureza distintas são descritos. Na Seção 5.4 apresentam-se as quinze métricas de similaridade entre strings consideradas e comparadas nos experimentos. Por fim, as configurações utilizadas na geração dos modelos de linguagem e as matrizes de dissimilaridade com base nas medidas de comparação de strings são detalhadas na Seção 5.5. 


\subsection{Módulos Desenvolvidos no VisPipeline}

Na Figura 5.2 apresenta-se a interface do VisPipeline. O layout divide-se em três subjanelas: a janela (a) apresenta uma lista de todos módulos disponíveis para gerar mapas visuais utilizando técnicas de projeção multidimensional e árvores filogenéticas, módulos para o pré-processamento de textos e também de imagens, ferramentas para a análise de projeções, medidas de distância, etc. A janela (b) é o espaço designado para a criação dos pipelines adicionando e unindo, na ordem desejada, os módulos listados em (a). Cada módulo tem diferentes tipos de parâmetros de entrada e saída, a união dos módulos deve respeitar a natureza dos parâmetros. Por fim, a janela (c) apresenta uma breve descrição dos módulos adicionados na janela (b), e relata também o estado da execução dos processos depois de que o botão Execute é acionado.

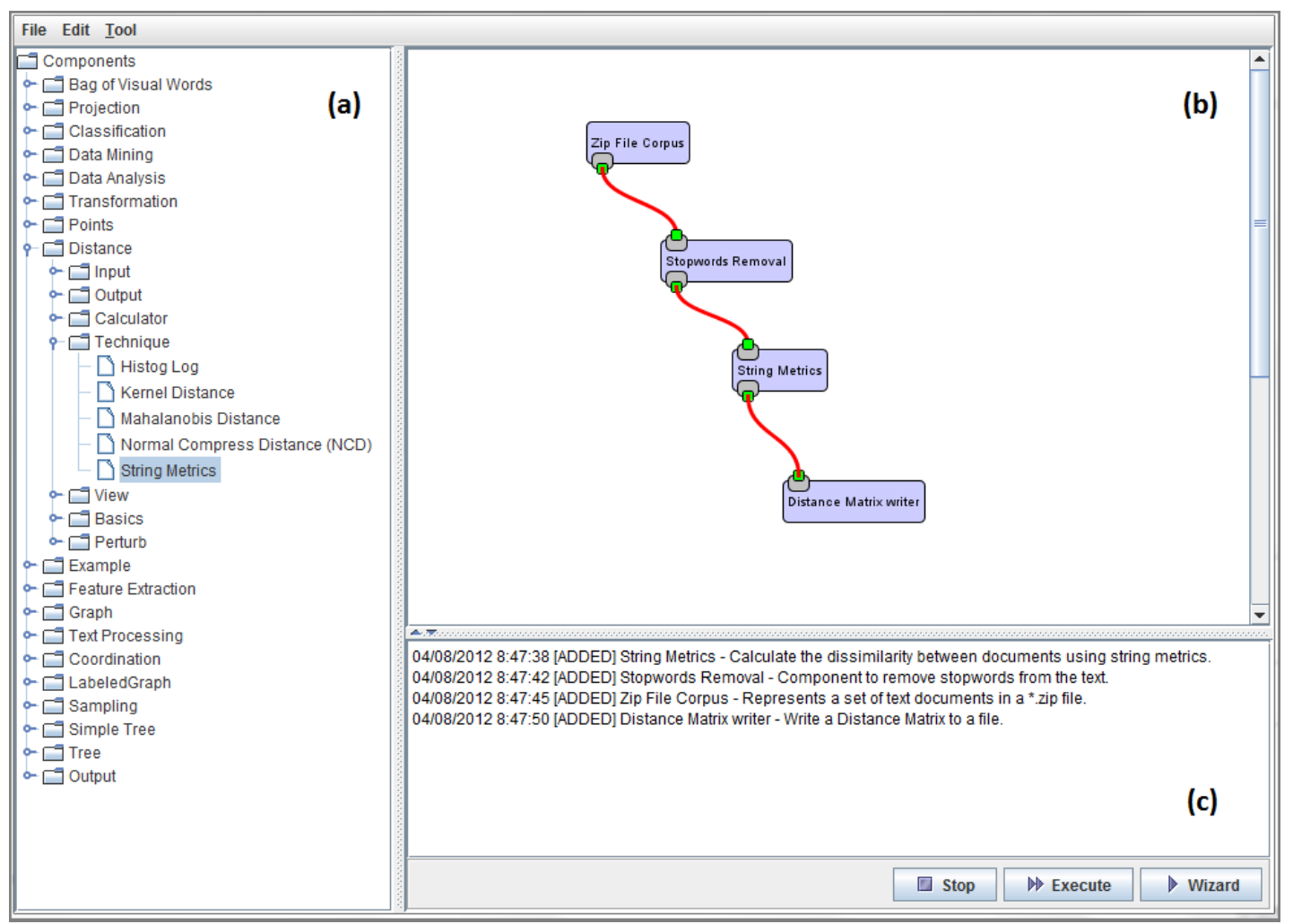

Figura 5.2: VisPipeline

Dois módulos foram incorporados à ferramenta neste trabalho: o primeiro computa o cálculo da dissimilaridade entre documentos utilizando medidas de comparação de strings. A Figura 5.3(a) apresenta um exemplo de pipeline utilizado para gerar uma matriz de distâncias de um conjunto de textos. A dissimilaridade é calculada usando métricas de comparação de strings (módulo String Metrics). O pipeline gerado é executado de 
forma descendente, começando o processo com a leitura do conjunto de textos (apresentado como um arquivo .zip). Em seguida, as palavras não relevantes podem ser removidas dos textos (este processo é opcional) e as stopwords podem ser especificadas dependendo da natureza do conjunto de textos. No módulo seguinte, a dissimilaridade entre documentos é calculada. O módulo inclui 13 diferentes métricas para o cálculo da dissimilaridade comparando strings textuais (ver Figura 5.3(b) e Seção 5.4). O pipeline finaliza com a criação de um arquivo contendo a matriz de distâncias com os valores de dissimilaridade entre todos os pares de documentos, que servirá como entrada para as técnicas de geração de mapas visuais.

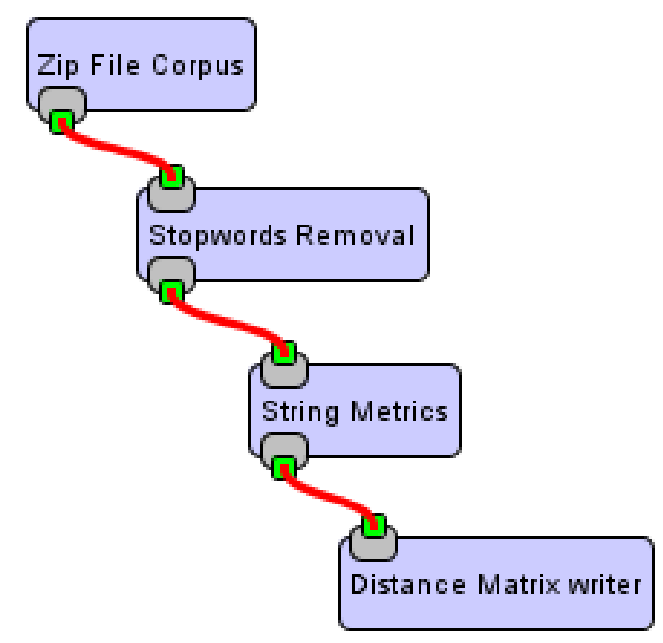

(a)

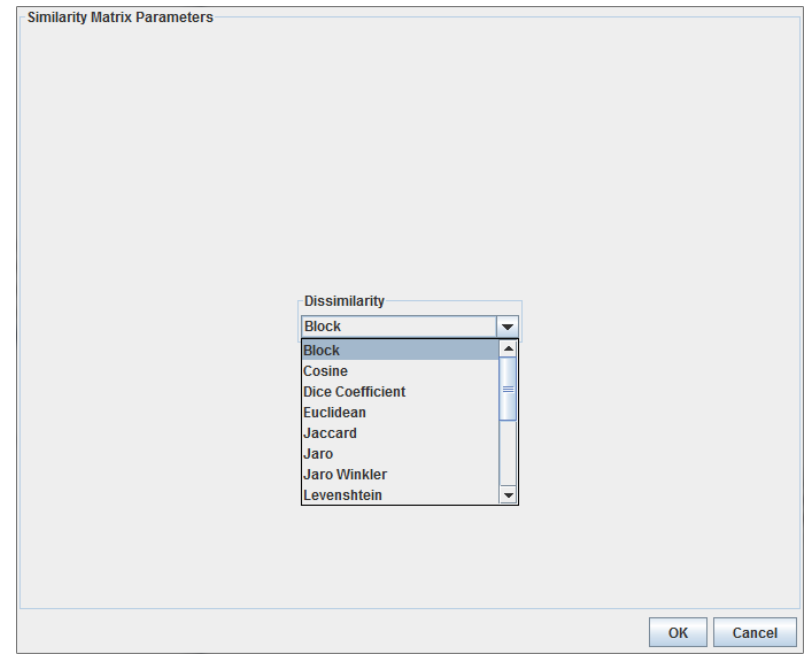

(b)

Figura 5.3: VisPipeline: Pipeline criado para computar uma matriz de dissimilaridade entre documentos, utilizando o módulo String Metrics.

O segundo módulo computa o iVSM para os documentos de uma coleção. O algoritmo é o mesmo utilizado na ferramenta incBoard (Pinho, 2009). Na Figura 5.4(a) apresenta-se o pipeline gerado para este fim. O processo começa com a leitura do conjunto de textos. No módulo iVSM, algumas configurações devem ser preenchidas (ver Figura 5.4(b)). Uma das ações necessárias é a especificação do modelo de linguagem a ser utilizado, o qual foi gerado anteriormente. Em seguida, valores do pré-processamento, tais como o algoritmo de lematização, o arquivo de stopwords e os valores dos cortes de Luhn (com opção de serem calculados de maneira automática), devem ser especificados. O módulo gera, para cada texto, um vetor de frequências de termos que nele ocorrem, aplicando as configurações anteriormente introduzidas. O pipeline finaliza com a criação de um arquivo 
contendo os valores de frequência para cada documento, que se utilizará como entrada para as técnicas de geração de mapas visuais.

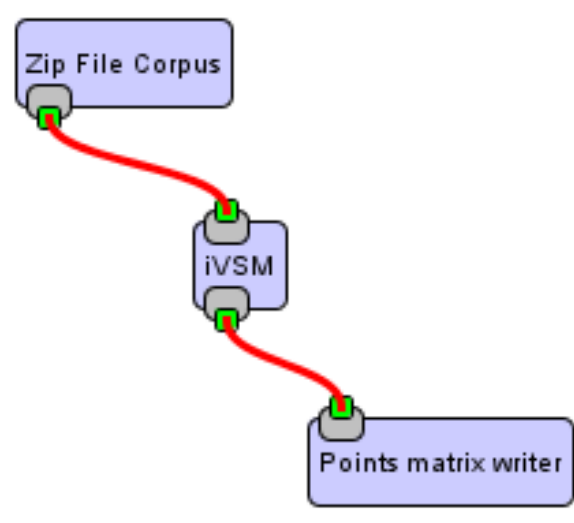

(a)

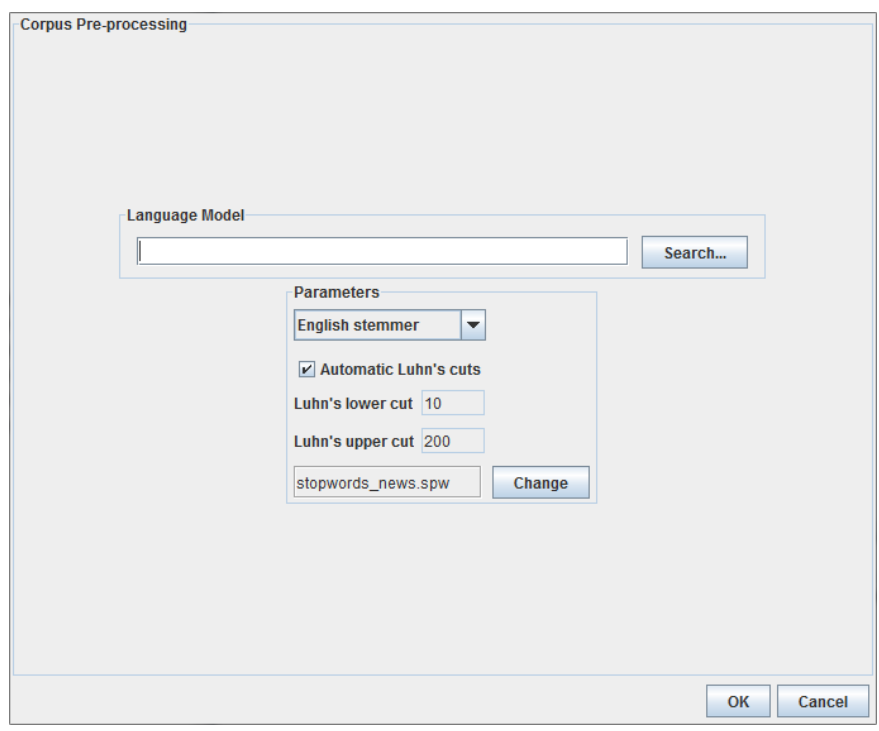

(b)

Figura 5.4: VisPipeline: Pipeline para computar do modelo iVSM dos documentos de uma coleção.

\subsection{Coleções de Textos}

A seguir são descritos os conjuntos de textos considerados nos experimentos: dois de notícias eletrônicas e um de artigos científicos.

\section{CBR-ILP-IR}

Este conjunto de textos consiste de 574 artigos científicos em inglês classificados em três áreas de pesquisa: (i) raciocínio baseado em casos (Case-Based Reasoning - CBR) com 276 documentos; (ii) programação em lógica indutiva (Inductive Logic Programming - ILP) com 119 documentos e (iii) recuperação de informação (Information Retrieval - IR) com 179 documentos. Ele foi construído agregando-se artigos obtidos de fontes associadas a cada uma dessas áreas, como anais de congressos especializados. Assim, os artigos CBR e ILP foram obtidos manualmente a partir do conjunto de notas Lecture Notes on Artificial Intelligence (LNAI), e os artigos IR foram coletados da internet. Para cada documento são considerados os seguintes atributos: título, autores, afiliação, resumo e referências 
bibliográficas. Este conjunto foi utilizado anteriormente para testes na integração de regras de associação e técnicas de projeção em mineração visual de textos (Lopes et al., 2007), para comparar técnicas de projeção (Paulovich et al., 2008), para avaliar métodos de agrupamento de dados (Almeida e Lopes, 2009) e técnicas de aprendizado ativo em tarefas de classificação (Motta et al., 2009). Está disponível na página http://infoserver. lcad.icmc.usp.br/infovis2/DataSets

\section{News2011}

Este conjunto de dados classificado News2011 for gerado neste trabalho como complemento aos existentes para a realização de testes com dados atuais. Foi desenvolvido um programa para coletar notícias fornecidas pelos feeds RSS dos provedores de notícias na Internet - Reuters, AP, BBC e CNN. A tecnologia RSS é utilizada para publicar conteúdo Web que muda ou é atualizado regularmente. Para isso, são utilizados feeds RSS que recebem estas atualizações e oferecem o conteúdo Web, ou resumos de conteúdo, juntamente com os links para as versões completas deste conteúdo. A Figura 5.5 apresenta um exemplo dos feeds RSS oferecidos por CNN.com e Reuters. Dos dados fornecidos pelos feeds RSS, foram considerados só o título e a descrição de cada notícia.

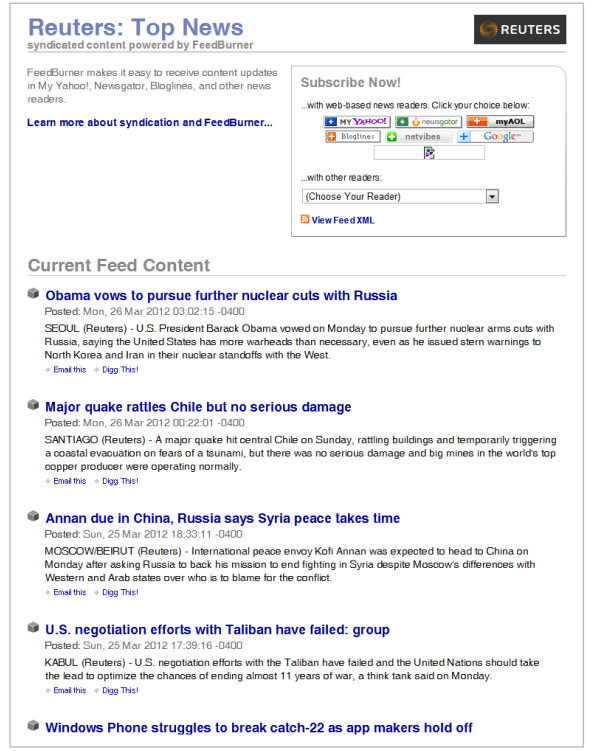

(a) Reuters

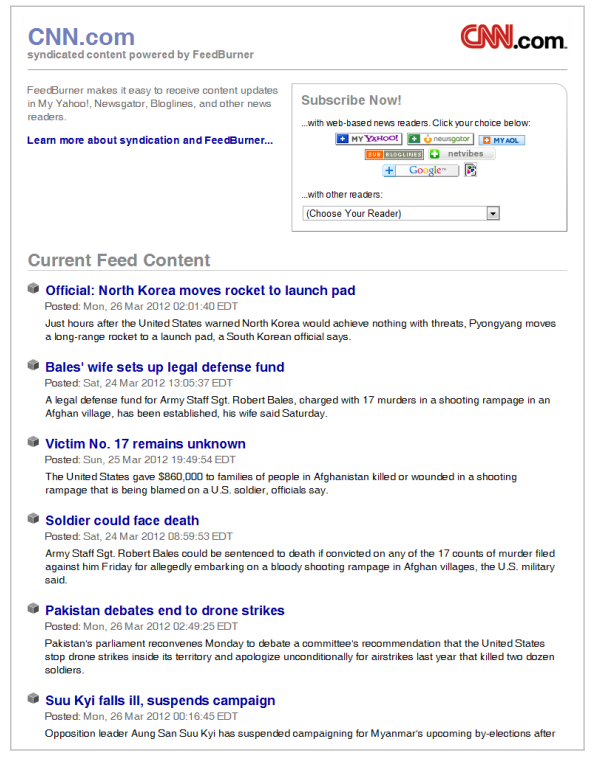

(b) CNN

Figura 5.5: As Figuras 5.5(a) e 5.5(a) apresentam um exemplo dos feeds RSS das notícias topo dos fornecedores de notícias Reuters e CNN.com, respectivamente. 
A coleta foi realizada durante 28 dias entre os meses de junho e julho de 2011 (duas semanas de cada), recuperando-se mais de 30.000 notícias dos quatro provedores. Desse grande conjunto foram escolhidas apenas as notícias com maior quantidade de documentos com um tema em comum. A escolha e classificação das notícias foram feitas manualmente, obtendo-se 1.771 documentos distribuídos em 23 classes diferentes. A Tabela 5.1 sumariza o conteúdo das 23 classes que compõem o conjunto, que está disponível no mesmo endereço do conjunto CBR-ILP-IR.

\begin{tabular}{|c|c|c|}
\hline Nome de classe & $\begin{array}{l}\text { Quantidade de } \\
\text { documentos }\end{array}$ & Descrição \\
\hline Afghanistan & 89 documentos & Conflitos no Afeganistão \\
\hline AmyWinehouse_death & 45 documentos & Morte de Amy Winehouse \\
\hline British_open & 35 documentos & Campeonato de golfe - British Open \\
\hline Bullet_train_China & 29 documentos & Acidente de trem na China \\
\hline E.coli_Europe & 93 documentos & Surto de epidemia de E. coli na Europa \\
\hline England_vs_India(sports) & 16 documentos & Competição de críquete (Lord's)- Inglaterra vs Índia \\
\hline Fukushima_Japan & 36 documentos & $\begin{array}{l}\text { Problemas na planta nuclear de Fukushima depois do } \\
\text { terremoto do Japão }\end{array}$ \\
\hline Greek_debt & 91 documentos & Crise econômica da Grécia \\
\hline Hacking_London & 336 documentos & Escândalo pelo hacking de telefone em Londres \\
\hline Heat_wave_USA & 17 documentos & $\begin{array}{l}\text { Notícias sobre a enorme onda de calor que atingiu grande } \\
\text { parte dos Estados Unidos }\end{array}$ \\
\hline Libya & 110 documentos & Conflitos na Líbia \\
\hline Mladic & 62 documentos & $\begin{array}{l}\text { Extradição do Ratko Mladic ao tribunal de crimes } \\
\text { de guerra das Nações Unidas }\end{array}$ \\
\hline Mumbai & 35 documentos & Explosão de três bombas em bairros lotados de Mumbai \\
\hline NASA & 34 documentos & Final da era do ônibus espacial - pousado do Atlantis \\
\hline NFL & 21 documentos & $\begin{array}{l}\text { - Votação dos proprietários da NFL para aprovação } \\
\text { do novo contrato com jogadores }\end{array}$ \\
\hline Norway & 166 documentos & $\begin{array}{l}\text { Atentado na Noruega - explosão de uma bomba e } \\
\text { tiroteios num acampamento }\end{array}$ \\
\hline President_Peru & 16 documentos & Eleições presidenciais no Peru \\
\hline Saline_contaminated & 13 documentos & Mortes por soro contaminado num hospital do Stockport \\
\hline Soma lia & 29 documentos & Notícias sobre a fome em áreas da Somália pela seca \\
\hline Syria & 102 documentos & Conflitos na Síria \\
\hline USA_crisis & 261 documentos & Crise econômica dos Estados Unidos \\
\hline Venezuelan_President & 26 documentos & Tratamento de câncer do presidente Chavez - Venezuela \\
\hline Yemen & 109 documentos & Conflitos governamentais no Iêmen \\
\hline
\end{tabular}

Tabela 5.1: Descrição do conjunto de dados News2011.

\section{NewsReuters}

A coleção Reuters-21578, disponibilizada em http://www . daviddlewis . com/resources/ testcollections/reuters21578, tem sido uma referência para trabalhos em avaliação e classificação texto (Apté et al., 1994a,b; Hayes et al., 1990) e outros mais recentes (Huh e Fienberg, 2012; Selvaraj et al., 2011; Subramanya e Bilmes, 2008; Yang e Lee, 2011). 
Esta é uma coleção de 21.578 notícias eletrônicas, inicialmente recolhidas e rotuladas pela Carnegie Group Inc. e Reuters Ltd. no decurso do desenvolvimento do sistema de categorização de texto CONSTRUE (Hayes e Weinstein, 1991). O conjunto Reuters21578 está distribuído em 22 arquivos, cada um com 1000 documentos (exceto o último, que contém 578 documentos). Os arquivos estão em formato SGML. As tags SGML são usadas para dividir cada notícia e cada documento em seções. Cada notícia está dividida nas seguintes categorias: data, notas ou observações, tópicos, lugares, pessoas, orgs, exchanges, empresas, unknown e texto que está dividido em autor, linha de tempo, título e corpo da notícia. Algumas das categorias podem não ter conteúdo.

NewsReuters é um subconjunto de 3.988 notícias da coleção Reuters-21578. Foram consideradas só aquelas notícias cuja categoria tópicos é identificada por uma única palavra (coffee com 114 documentos, crude com 354 documentos, 2.686 documentos para o tópico earn, 210 documentos para o tópico interest, ship com 156 documentos, sugar com 135 documentos e finalmente trade com 333 documentos) pela qual foram classificadas. Para as notícias selecionadas, foram considerados apenas o conteúdo dos atributos título e corpo da categoria texto, para gerar o modelo vetorial.

\subsection{Medidas para o Cálculo da Similaridade entre Strings}

Nos experimentos descritos no Capítulo 6 utilizamos quinze métricas para o cálculo de similaridade entre strings, disponibilizadas pela biblioteca de código aberto SimMetrics ${ }^{123}$ : distância de block, similaridade do Cosseno, coeficiente de Dice, distância Euclidiana, distância de Jaccard, distância de Jaro, distância de JaroWinkler, distância de Levenshtein, Coeficiente de Matching, distância de MongeElkan, distância de NeedlemanWunch, Coeficiente de Overlap, Qgram, distância de SmithWaterman, distância de Soundex. Na implementação da biblioteca todas as métricas calculam a similaridade entre duas strings; logo, para transformar essa medida em uma medida de dissimilaridade basta utilizar o seu complemento. Na Figura 5.6 apresenta-se o gráfico dos tempos de processamento para calcular a similaridade entre duas strings do mesmo tamanho, para as diferentes métricas. Considerando diferentes tamanhos da string, definido pela quantidade de tokens que a compõe, o eixo $X$ informa os tamanhos e o eixo $Y$ informa o tempo para processar a similaridade entre elas (em segundos). Observa-se na Figura 5.6(a) que, quanto maior o

\footnotetext{
${ }^{1}$ http://sourceforge.net/projects/simmetrics/

${ }^{2}$ http://staffwww.dcs.shef.ac.uk/people/S.Chapman/stringmetrics.html (deadlink)

${ }^{3}$ http://web.archive.org/web/20070110010145/http://www.dcs.shef.ac.uk/ sam/ stringmetrics.html
} 
tamanho da string, mais demorado torna-se o cálculo da similaridade, sendo que a distância MongeElkan mostra-se como a mais demorada. Na Figura 5.6(b) apresenta-se os gráficos da Figura 5.6(a), sem o gráfico da distância MongeElkan. Das métricas restantes, SmithWaterman, Qgram, Levenshtein, Jaro e JaroWinkler resultaram como as mais demoradas.

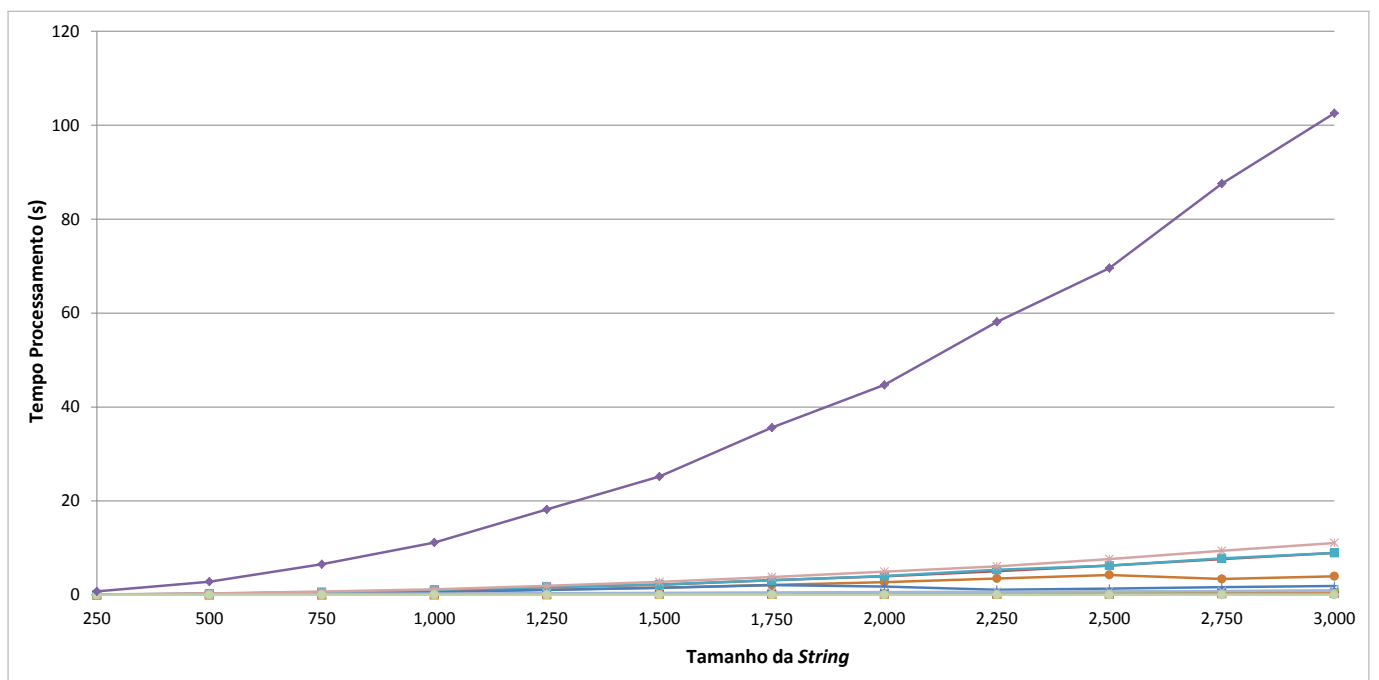

(a)

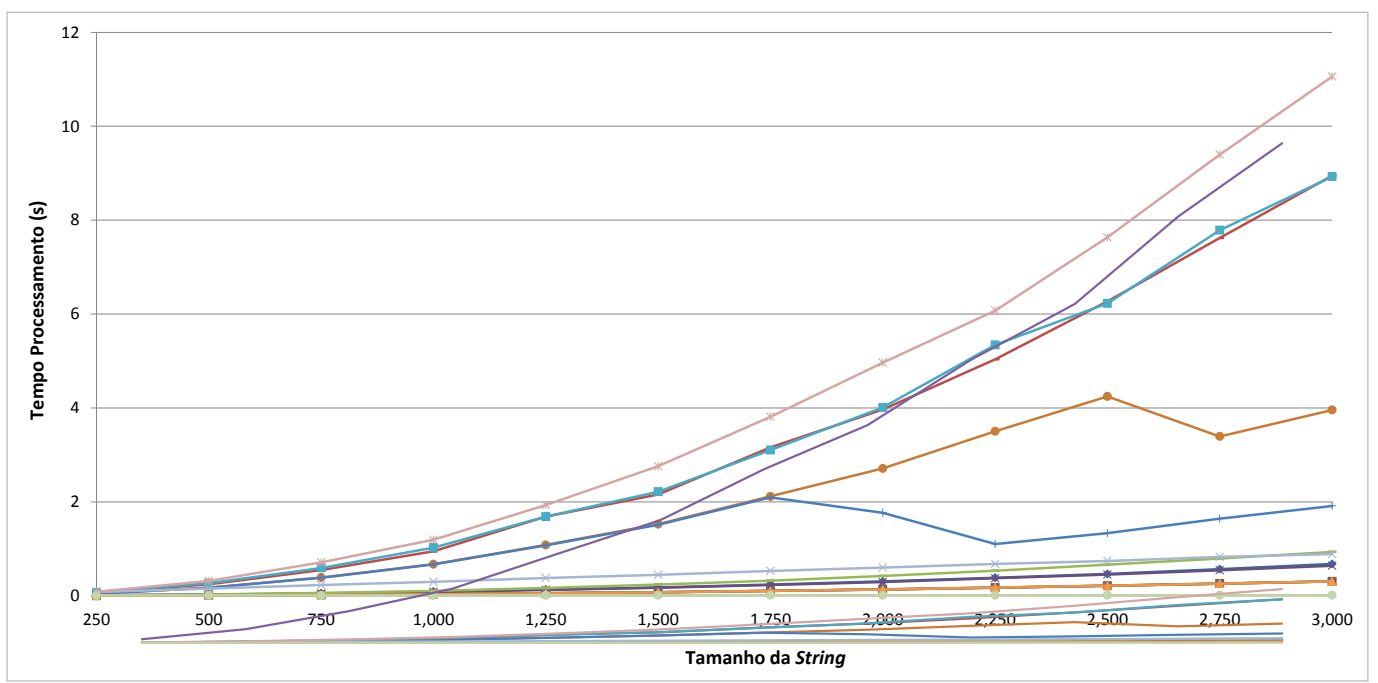

(b)

\begin{tabular}{|c|c|c|c|c|}
\hline$\rightarrow$ MongeElkan & $\rightarrow$ Block & $\#$-Cosseno & $₫$ Coef. Dice & *Euclidiana \\
\hline * Jaccard & $\rightarrow$ Jaro & +JaroWinkler & -Levenshtein & -Coef. Matching \\
\hline -N-NeedlemanWunch & $\pitchfork$ Coef. Overlap & - Qgram & \#SmithWaterman & $\rightarrow$ Soundex \\
\hline
\end{tabular}

Figura 5.6: Tempos de processamento de diferentes medidas de comparação de strings, no cálculo da dissimilaridade entre duas strings de igual tamanho. A Figura 5.6(a) apresenta os gráficos gerados para as quinze métricas utilizadas. A Figura 5.6(b) apresenta o gráfico anterior, excluindo a medida MongeElkan. 


\subsection{Configuração dos Experimentos}

Nesta seção são apresentados detalhes sobre a geração dos modelos de linguagem usados no cálculo do modelo vetorial incremental nos experimentos. Ainda nesta seção apresentase detalhes do pré-processamento dos textos na abordagem de geração de matrizes de distância por meio de comparação de strings.

\subsubsection{Configuração do Modelo de Linguagem}

Dois modelos de linguagem, para conjuntos de textos de natureza distintos, foram gerados para serem utilizados nos experimentos:

1. Modelo de Linguagem para artigos científicos: Para gerar este modelo de linguagem foi utilizada uma coleção de documentos conhecida como all, utilizada anteriormente para comparar técnicas de projeção em (Paulovich et al., 2008) e disponível em http://infoserver.lcad.icmc.usp.br/infovis2/DataSets. Esta coleção consiste de 2.814 artigos científicos em inglês, divididos em 6 classes definidas segundo a procedência do artigo. Para cada documento foram considerados os seguintes atributos: título, autores, afiliação, resumo e referências bibliográficas. A Tabela 5.2 sumariza as configurações utilizadas para gerar o modelo vetorial desse conjunto de dados. Uma vez gerado o modelo vetorial, procede-se na construção do modelo de linguagem como foi detalhado na Seção 3.2.2. Logo, este modelo de linguagem atuará como uma representação inicial aproximada de alguma coleção de artigos científicos desconhecida.

\begin{tabular}{|c|c|}
\hline Estratégia de pré-processamento & Valores \\
\hline \hline Remoção de termos irrelevantes & sim, incluindo termos específicos \\
& utilizados nesse tipo de documento \\
Redução dos termos a seus radicais & não \\
Cortes de Luhn & não \\
Medida de frequência & TF-IDF \\
\hline
\end{tabular}

Tabela 5.2: Configuração utilizada na geração do modelo vetorial para o conjunto de dados all

2. Modelo de Linguagem para notícias: Para gerar o modelo de linguagem para documentos que representam notícias foi utilizada uma coleção de documentos conhecida como AP_BBC_CNN_Reuters disponível em http://infoserver. lcad.icmc.usp.br/infovis2/DataSets. Este conjunto de dados inclui 2.684 artigos jornalísticos em inglês disponibilizados na Internet em formato RSS pelas 
agências Reuters, BBC, CNN e Associated Press. Estes artigos foram coletados durantes dois dias em Abril de 2006. Para cada documento são considerados os atributos título e descrição. A Tabela 5.3 sumariza as configurações utilizadas para gerar o modelo vetorial desse conjunto de dados. Novamente, uma vez gerado o modelo vetorial, procede-se na construção do modelo de linguagem, que atuará como representação inicial aproximada de uma coleção de notícias desconhecida.

\begin{tabular}{|c|c|}
\hline Estatégia de pré-processamento & Valores \\
\hline \hline Remoção de termos irrelevantes & sim, incluindo termos específicos \\
& utilizados nesse tipo de documento \\
Redução dos termos a seus radicais & não \\
Cortes de Luhn & não \\
Medida de frequência & TF-IDF \\
\hline
\end{tabular}

Tabela 5.3: Configuração utilizada na geração do modelo vetorial para o conjunto de dados AP_BBC_CNN_Reuters

\subsubsection{Configuração das Medidas de Similaridade de Strings}

Conforme exposto anteriormente, uma grande vantagem de usar métricas sobre strings é que elas não requerem estratégias de pré-processamento, como acontece no modelo do espaço vetorial. No entanto, o cálculo destas distâncias tem, em geral um alto custo computacional, pois o tempo de processamento está diretamente relacionado ao tamanho das strings. Assim, quanto maior esse tamanho, maior o tempo de processamento no cálculo das dissimilaridades. Na tentativa de reduzir o tamanho das strings processadas, termos irrelevantes presentes foram removidos antes realizar o cálculo das dissimilaridade. A métrica Qgram utiliza $q=3 \mathrm{em}$ todos os experimentos. Com o objetivo de investigar o efeito de aplicar essa estratégia nos resultados, layouts visuais foram gerados utilizando as técnicas LSP e NJ sobre as coleções CBR-ILP-IR e News2011. As Figuras 5.7 e 5.8 apresentam alguns dos resultados de precisão (NH) (no intervalo de um a trinta vizinhos mais próximos) de quatro métricas diferentes sobre as coleções CBR-ILP-IR e News2011, respectivamente. Enquanto aos parâmetros da LSP, para o conjunto CBR-ILP-IR foram utilizados 57 pontos de controle e 15 vizinhos mais próximos e, para o conjunto News2011, utilizamos 177 pontos de controle e 15 vizinhos mais próximos. Note-se que, de maneira geral, a precisão melhora quando termos irrelevantes, nas strings, são removidos. Por outro lado, observamos alguns comportamentos diferenciados, como é o caso dos gráficos de NH das Figuras 5.7(a) e 5.7(b), correspondentes à similaridade do Cosseno em que a remoção de stopwords foi favorável para a projeção LSP, mas não para a árvore NJ. Assim, 
a Tabela 5.4 sumariza a configuração utilizada para cada métrica, com base nos testes de precisão segundo a NH, visando obter o melhor desempenho qualitativo das métricas de comparação de strings no cálculo da matriz de dissimilaridades nos três conjuntos de textos: CBR-ILP-IR, News2011 e NewsReuters.

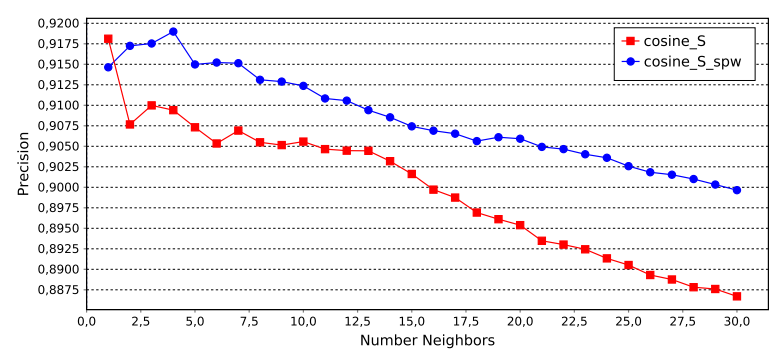

(a) Cosseno (strings) com LSP

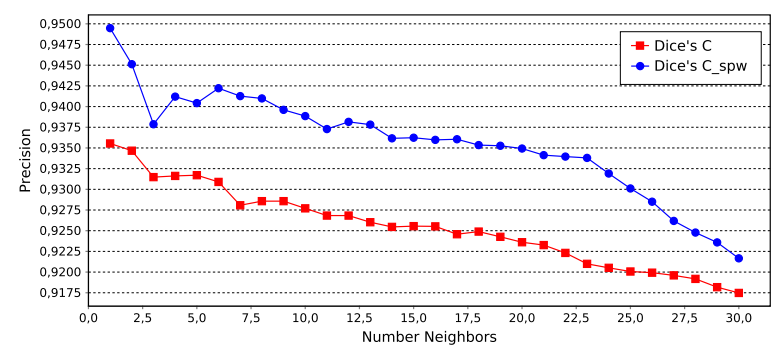

(c) Coeficiente de Dice com LSP

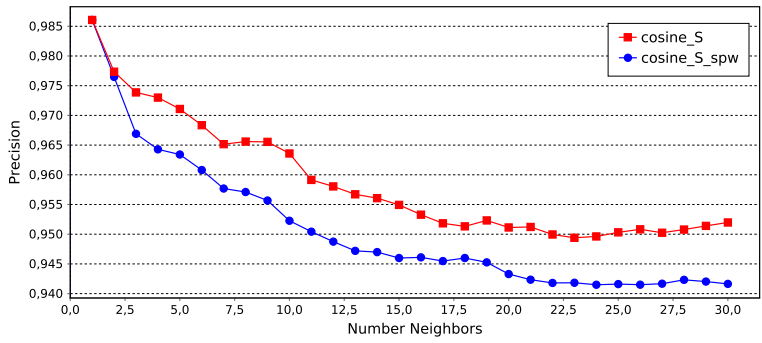

(b) Cosseno (strings) com NJ

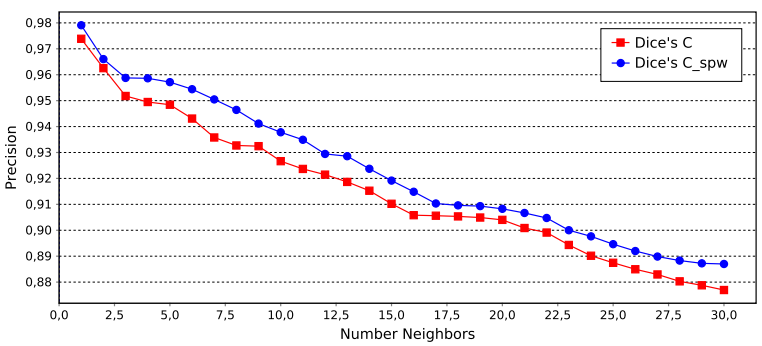

(d) Coeficiente de Dice com NJ

Figura 5.7: Gráficos de precisão para o conjunto CBR-ILP-IR

Quanto aos tempos de processamento na geração da matriz de dissimilaridade, sabese que as métricas de comparação de strings são mais demoradas do que o cálculo da similaridade do cosseno sobre os modelos vetoriais. A Figura 5.9 apresenta os tempos de processamento para todas as métricas testadas, incluindo a NCDs. As matrizes de dissimilaridade foram geradas utilizando um computador com dois processadores Hexa Core Intel(R) Xeon(R) X5690 de 3,46GHz e 32GB de memoria RAM.

Esperava-se que, aplicando a estratégia de remoção de stopwords, os tempos de processamento fossem reduzidos em todos os casos, mas como observamos na Figura 5.9, isso não acontece sempre. É o caso, por exemplo, do conjunto de textos CBR-ILP-IR: das quinze métricas apresentadas, só em duas (Jaro e JaroWinkler) o tempo de processamento foi reduzido com a remoção prévia de stopwords. Esse comportamento não acontece nos outros dois conjuntos, em que só quatro métricas não apresentaram esta melhora: as métricas Qgram e NCDs no conjunto News2011 e Levenshtein, NCDs e Soundex no conjunto NewsReuters. 


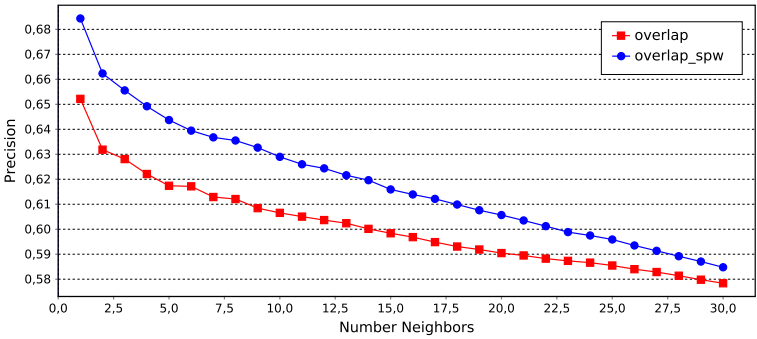

(a) Coeficiente de Overlap com LSP

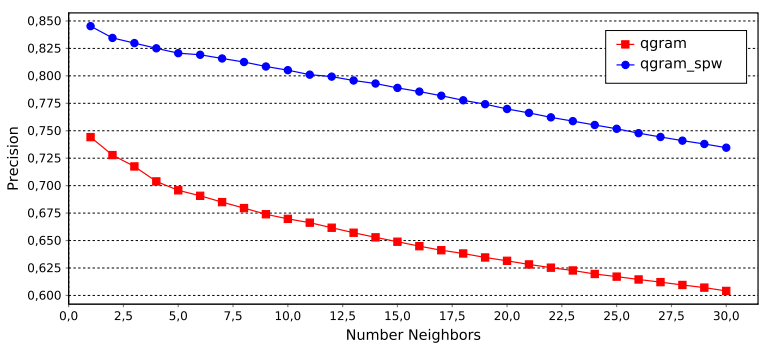

(c) QGram com LSP

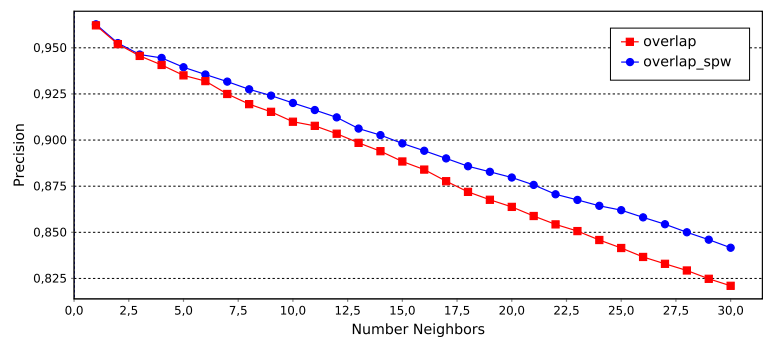

(b) Coeficiente de Overlap com NJ

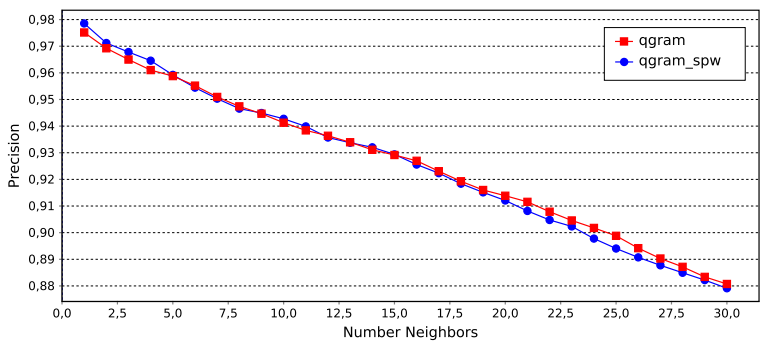

(d) QGram com NJ

Figura 5.8: Gráficos de precisão para o conjunto News2011

\subsection{Considerações Finais}

Neste Capítulo foram apresentados os módulos desenvolvidos para conduzir nossos experimentos. Os módulos foram desenvolvidos na linguagem de programação Java e introduzidos na ferramenta VisPipeline. Os conjuntos de textos, assim como as métricas de comparação de strings, utilizados nos experimentos foram descritos. Também foram apresentados detalhes sobre geração dos modelos de linguagem, assim como sobre as configurações empregadas no pré-processamento dos textos, anteriormente ao cálculo das dissimilaridades, nas métricas baseadas em strings.

No próximo capítulo são apresentados os resultados obtidos no estudo comparativo que considera diferentes layouts gerados com várias métricas de comparação de strings e com os modelos VSM e iVSM e a distância do cosseno. 


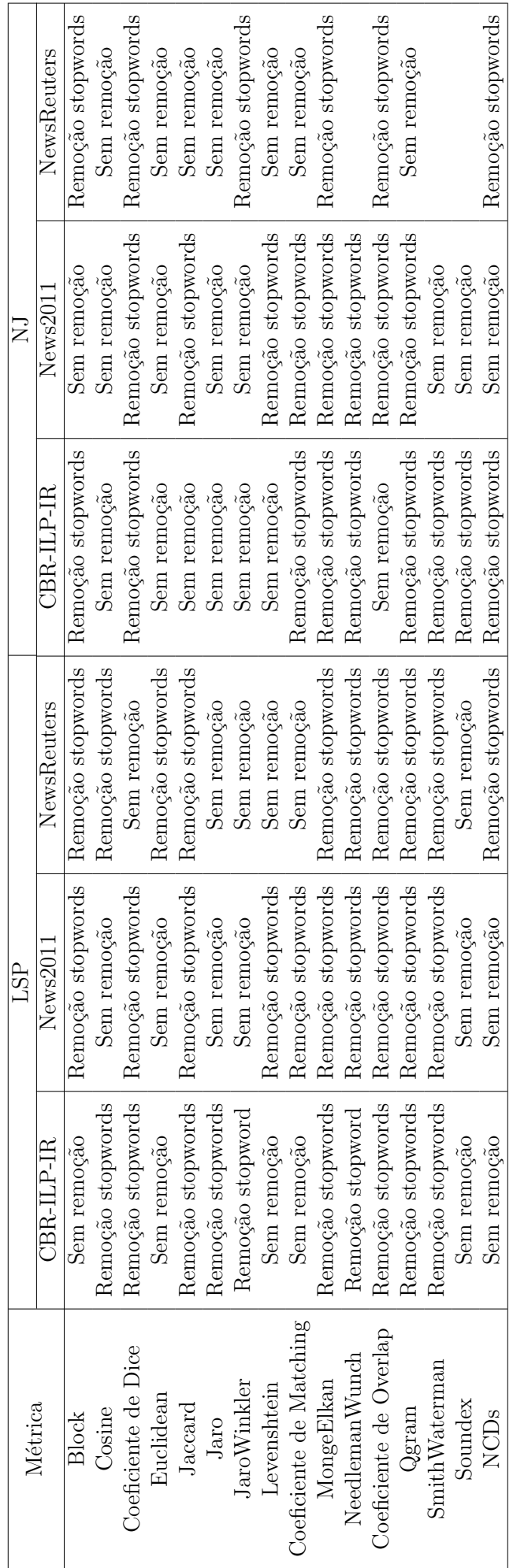

Tabela 5.4: Pré-processamento aplicado (remoção de stopwords) aos textos previamente ao cómputo das métricas de comparação de strings. 


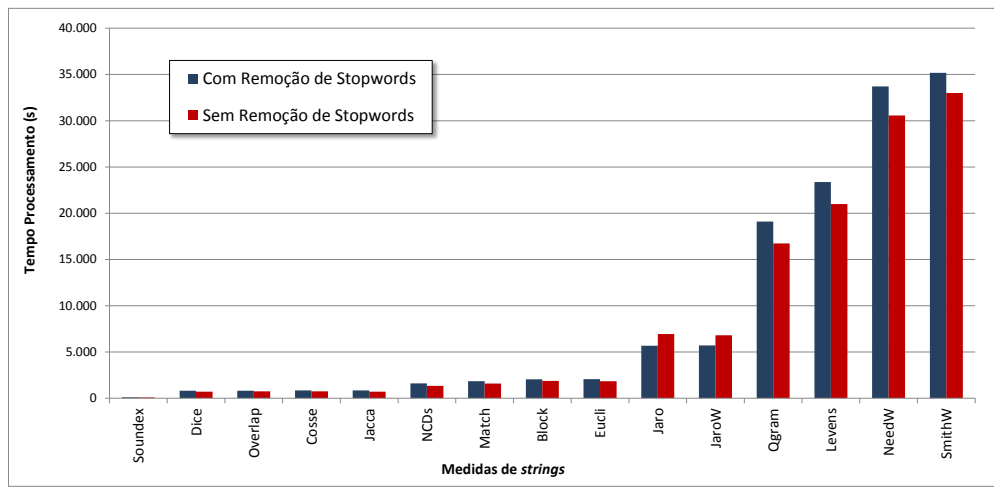

(a) Conjunto CBR-ILP-IR

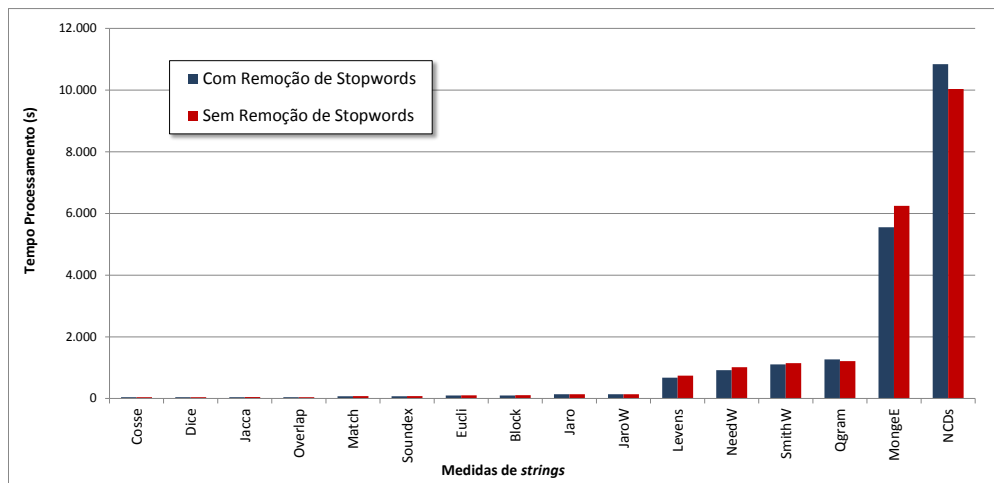

(b) Conjunto News2011

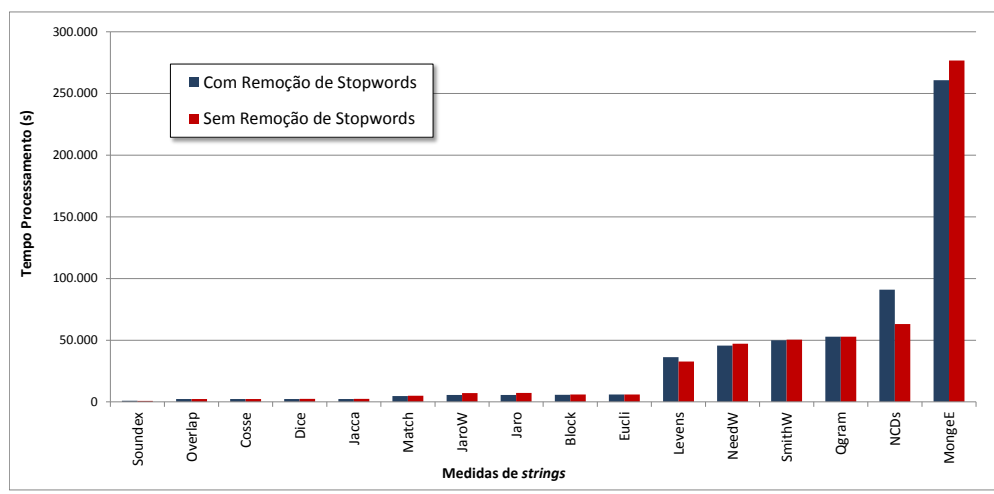

(c) Conjunto NewsReuters

Figura 5.9: Tempos de processamento em segundos, para o cálculo da matriz de dissimilaridade utilizando diferentes métricas de comparação de strings com e sem remoção de stopwords. 


\subsection{Considerações Iniciais}

Esse capítulo descreve os experimentos computacionais realizados com a finalidade de analisar layouts gerados por matrizes de dissimilaridade computadas com métricas de comparação de strings e com a distância do cosseno sobre o modelo VSM convencional e sobre o modelo iVSM.

Os experimentos foram divididos em três etapas, cada qual com objetivos diferentes. As próximas seções são dedicadas a apresentar alguns cenários de análise de conjuntos textuais de diferentes domínios utilizando a ferramenta VisPipeline e os resultados obtidos. Na Seção 6.2 comparamos a capacidade de preservação de vizinhança nos layouts obtidos com matrizes de similaridade computadas com diferentes métricas de comparação de strings. A Seção 6.3 apresenta uma comparação visual dos layouts obtidos com a LSP e com a NJ, usando como entrada matrizes de dissimilaridade computadas empregando a distância do cosseno sobre representações VSM e iVSM, respectivamente. Os gráficos de precisão NH para os layouts também são comparados. Por fim, com base na análise comparativa conduzida no primeiro experimento, os layouts das cinco medidas com os melhores resultados de precisão NH foram selecionados, e comparados com layouts obtidos com matrizes de dissimilaridade computadas com a NCDs e a distância do cosseno 
sobre os modelos VSM e iVSM. Os resultados do estudo comparativo são apresentados na Seção 6.4.

\subsection{Experimento 1: Comparando Medidas sobre Strings}

Nesse primeiro experimento, quinze medidas apresentadas na Seção 5.4 são comparadas quanto à capacidade de preservação da vizinhança em layouts visuais construídos para dois conjuntos de textos (CBR-ILP-IR e News2011), utilizando LSP e árvores NJ.

As matrizes de dissimilaridade para todas as métricas foram computadas e projetadas utilizando a LSP (consideramos duas configurações distintas nos parâmetros da LSP, mudando o número de pontos de controle e o número de vizinhos) e a NJ. Os mapas gerados foram avaliados pela qualidade visual e também comparando suas correspondentes curvas NH. A Figura 6.1 apresenta três gráficos de precisão relativos ao conjunto de textos CBR-ILP-IR com a árvore NJ e com duas configurações da projeção LSP. Para cada métrica um layout foi gerado e sua curva de precisão NH computada, para serem comparadas.

Foram utilizados 57 pontos de controle e 15 vizinhos como parâmetros da técnica LSP, nas projeções cujas curvas NH são ilustradas na Figura 6.1(a). Esses valores são definidos pela LSP como ótimos, onde o número de pontos de controle é um valor próximo a $10 \%$ da quantidade de documentos, e o número de vizinhos é um valor entre 10 e 15 (Paulovich, 2008). De modo geral, dois grandes grupos de curvas podem ser identificados. Na parte superior, encontram-se as curvas NH das projeções que melhor caracterizam as classes existentes no conjunto de textos (valores de precisão acima de 0,78 ), sendo que o layout gerado com a matriz de dissimilaridade computada pelo Coeficiente de Dice alcançou os melhores resultados, com valores de precisão próximos a 0,95 (sendo o valor máximo 1,0). Na parte inferior do gráfico, com valores de precisão abaixo do 0,6 , encontramse as projeções que visivelmente foram as piores e, portanto, identificadas como menos precisas, entre elas as computadas com as métricas JaroWinkler e Soundex. Uma segunda configuração nos parâmetros de entrada da LSP foi utilizada, com 177 pontos de controle e número de vizinhos igual a 15, com o objetivo de estudar o seu efeito no layout final. Com essa nova configuração novas projeções foram geradas e os resultados de precisão são apresentados na Figura 6.1(b).

Com essa nova configuração, algumas medidas apresentaram melhor desempenho em relação a anterior, é o caso dos mapas visuais computadas com matrizes de dissimilaridade obtidas com a distância de Jaccard, Cosseno e Coeficiente de Overlap, os que ficaram entre 
os mais precisos, junto com o Coeficiente de Dice. Mas também foram observados resultados piores, por exemplo, as métricas Qgram e SmithWaterman, cujos valores de precisão foram menores em relação aos apresentados no gráfico anterior. Observa-se também que, como observado no gráfico anterior, as métricas JaroWinkler e Soundex resultaram sendo as menos precisas.

A tarefa de definir a configuração adequada para obter projeções com os melhores resultados visuais e melhor precisão torna-se difícil e subjetiva. Esse problema não existe na árvore NJ, em que nenhuma configuração precisa ser feita, o que representaria uma vantagem sobre técnicas paramétricas, como a LSP. A Figura 6.2(c) mostra o gráfico NH das árvores NJ geradas para o mesmo conjunto de artigos científicos. Note-se que, novamente, as métricas que apresentaram os piores resultados foram a JaroWinkler e Soundex. Neste caso, a matriz de dissimilaridade calculada com o Coeficiente de Overlap apresentou os melhores resultados com valores de precisão entre 0,965 e 1,0. O grupo das melhores inclui também as medidas Qgram e a do Cosseno.

Gráficos de precisão para projeções LSP e árvores NJ foram geradas também para o conjunto de documentos News2011, apresentados na Figura 6.2. Neste caso, as configurações utilizadas nas projeções LSP foram: (a) 177 pontos de controle e número de vizinhos igual a 15 (Figura 6.2(a)); e (b) 150 pontos de controle e número de vizinhos igual a 20 (Figura 6.2(b)). Nas duas configurações, a métrica com menor precisão foi a Jaro, com valores em torno de 0,2 (lembrando que o máximo é 1,0) e a que apresentou melhores resultados foi a Qgram, com valores entre 0,75 e 0,82. Já com a NJ, os valores de precisão melhoram para todas as métricas: a Qgram continua em primeiro lugar, com valores entre 0,89 e 0,96, seguida de perto pelas métricas do Cosseno, Coeficiente de Matching, Coeficiente de Dice, Coeficiente de Overlap, Jaccard, SmithWaterman e Block.

Os gráficos NH gerados para cada conjunto de textos revelam que as projeções e árvores geradas com matrizes de dissimilaridade construídas usando as métricas JaroWinkler, Jaro, Soundex e NeedlemanWunch são as que visivelmente produziram os piores resultados, i.e., mostraram-se menos precisas. Aquelas que apresentaram melhores resultados, preservando melhor os agrupamentos das classes definidas nos conjuntos de textos foram: Coeficiente de Matching, Coeficiente de Dice, Coeficiente de Overlap, Jaccard, Block, Cosseno e QGram, sendo que a vantagem dependente do conjunto de textos. Dessa forma, cinco dessas métricas que parecem as mais promissoras foram escolhidas para experimentos posteriores: Qgram, Coeficientes de Overlap, Coeficientes de Dice, o Coeficiente de Matching e Cosseno a qual, desde este ponto, é identificada como cosseno_S, para 


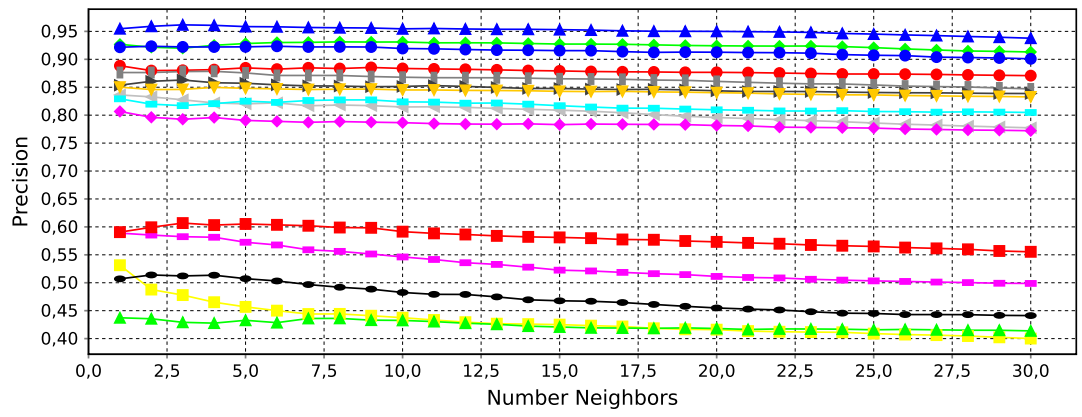

- euclidean $\rightarrow$ cosine $\mathrm{S} \rightarrow$ jaroWinkler $\rightarrow$ levenshtein - matching's $\mathrm{C}-$ mongeElkan

$\rightarrow$ needlemanWunch $\rightarrow$ overlap's C $\Vdash$ qgram $\leftarrow$ smithWaterman - soundex $\rightarrow$ block $₫$ dice's C

$\rightarrow$ jaccard - - jaro

(a) LSP $57-15$

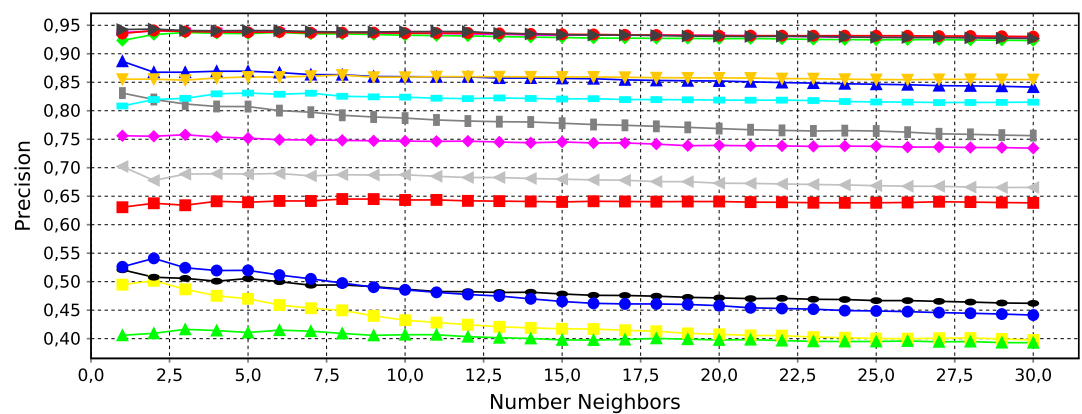

- euclidean - jaro $\rightarrow$ jaroWinkler $\rightarrow$ levenshtein - matching's $\mathrm{C}-$ mongeElkan

- needlemanWunch $\rightarrow$ overlap's $\mathrm{C} \Vdash$ qgram $\leftarrow$ smithWaterman - soundex $\rightarrow$ dice's $\mathrm{C} \star$ block

$\rightarrow$ jaccard - - cosine_s

(b) LSP 177-15

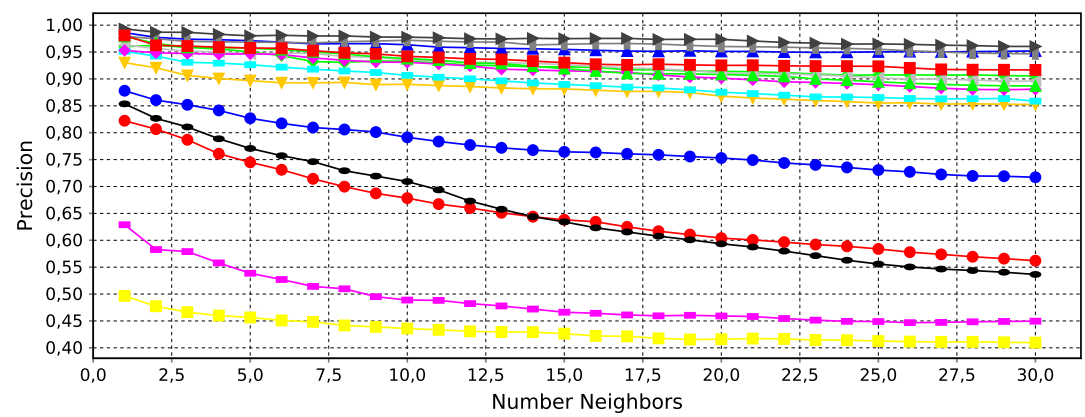

- - jaccard $\bullet$ euclidean - dice's $C \leftrightarrow$ - levenshtein - matching's $C \rightarrow$ mongeElkan

$\rightarrow$ needlemanWunch $\rightarrow$ overlap's $C \rightarrow$ qgram $\rightarrow$ smithWaterman - soundex $\rightarrow$ jaro $\neq$ cosine_S

$\rightarrow$ block - - jaroWinkler

(c) NJ

Figura 6.1: Resultados de precisão das projeções do conjunto de artigos científicos CBRILP-IR

diferenciá-la com a métrica do cosseno utilizada para o cálculo da dissimilaridade nos modelos vetoriais. 


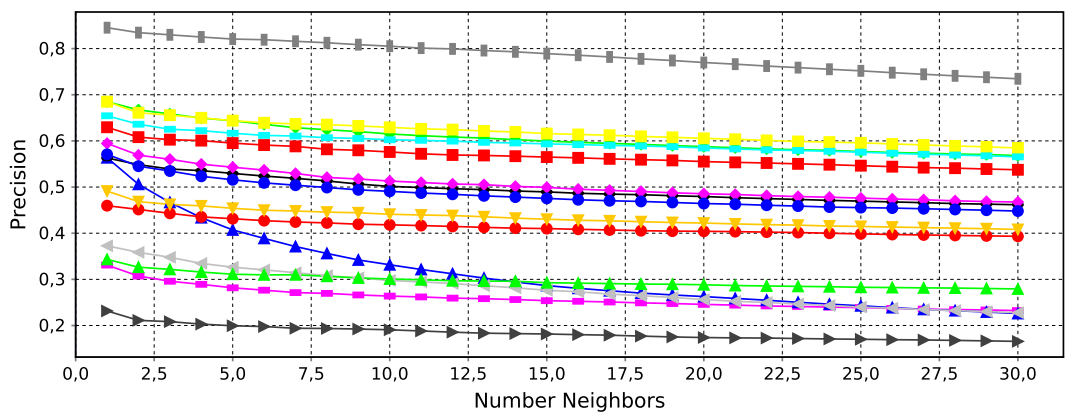

- block $\bullet$ dice's C $\rightarrow$ euclidean $\rightarrow$ jaccard - cosine_S $\rightarrow$ mongeElkan $\rightarrow$ matching's $C \rightarrow$ jaro

- qgram $\leftarrow$ jaroWinkler - overlap's $\mathrm{C} \rightarrow$ levenshtein $₫$ soundex $\leftrightarrow$ smithWaterman

- needlemanWunch

(a) LSP 177-15

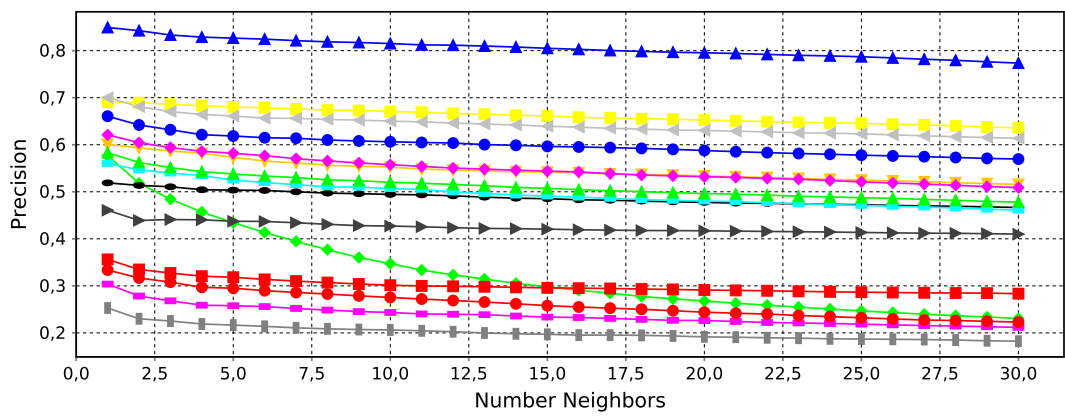

- - euclidean $\rightarrow$ - block $\rightarrow$ jaccard $\rightarrow$ dice's C - - cosine_S $\rightarrow$ matching's C $\rightarrow$ mongeElkan

$\rightarrow$ levenshtein $\rightarrow$ jaro $\nleftarrow$ smithWaterman - overlap's $\mathrm{C} \rightarrow$ jaroWinkler $\rightarrow$ qgram $\rightarrow$ soundex

- needlemanWunch

(b) LSP 150-20

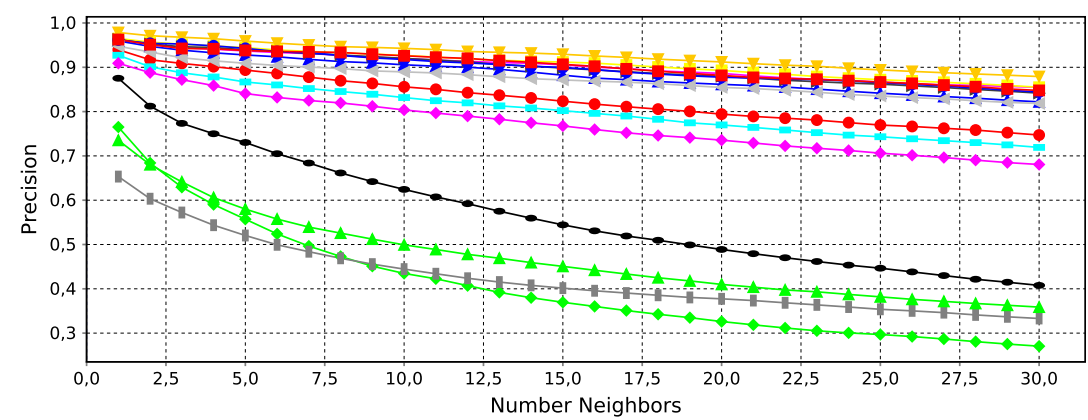

- - jaccard $\bullet$ block $\rightarrow$ jaroWinkler $\bullet$ mongeElkan - levenshtein $\rightarrow$ qgram $\bullet$ needlemanWunch

$\rightarrow$ overlap's $\mathrm{C} \Vdash$ soundex $\rightarrow$ smithWaterman - dice's $\mathrm{C} \rightarrow$ euclidean $₫$ matching's $\mathrm{C} \rightarrow$ jaro

- - cosine_S

(c) NJ

Figura 6.2: Resultados de precisão das projeções do conjunto de notícias News2011 


\subsection{Experimento 2: Comparando o VSM e iVSM}

O estudo a seguir busca verificar como a iVSM se adapta à tarefa de representar documentos como um vetor de frequências, e como se compara com o Vector Space Model (VSM) convencional.

Como foi explicado em capítulos anteriores, o iVSM será construído com base nos termos presentes no modelo de linguagem. Assim, as Figuras 6.3 e 6.4 buscam ilustrar o comportamento do modelo vetorial incremental, conforme novos documento são adicionados.

A Figura 6.3 apresenta um ranking das frequências dos cinco termos mais frequentes em um determinado momento, durante a construção do modelo vetorial incremental para o conjunto CBR-ILP-IR. O eixo horizontal refere-se ao momento temporal, representado pelo número de documentos adicionados até esse ponto; e o eixo vertical indica a porcentagem de documentos que incluiu o termo expresso. A fim de entender melhor a representação, consideramos apenas as frequências dos cinco termos mais frequentes e seu porcentual relativo, a medida que a coleção é 'construída' (simulando um cenário incremental). No instante de tempo um, ou seja, depois de adicionado o primeiro documento, as cinco palavras mais frequentes no modelo vetorial incremental foram: data: $\mathrm{DF}=1.436$ (21,86\%), system: $\mathrm{DF}=1.223(18,62 \%)$, computer: $\mathrm{DF}=1.325$ (20,17\%), visualization: $\mathrm{DF}=1.316(20,03 \%)$ e science $: \mathrm{DF}=1.269(19,32 \%)$. No próximo momento considerado, depois de adicionados cem documentos, os mesmos termos se mantem no ranking, mas algumas frequências mudaram: data: $\mathrm{DF}=1.461(21,85 \%)$, system: $\mathrm{DF}=1.314(19,65 \%)$, computer: $\mathrm{DF}=1.326(19,83 \%)$, visualization: $\mathrm{DF}=1.316(19,68 \%)$ e science: $\mathrm{DF}=1.269$ $(18,98 \%)$. Note que o termo science não incrementou sua frequencia, pelo que, no terceiro momento, ele não é mais um dos cinco mais frequentes, sendo substituído pelo termo information: $\mathrm{DF}=1.976$ (26,3\%). Finalmente, esses cinco termos foram mantidos até a coleção ter 500 documentos adicionados.

A Figura 6.4 apresenta, nos mesmos moldes, um ranking da frequências dos cinco termos mais frequentes durante a construção do modelo vetorial incremental para o corpus News2011. Note-se que nesse caso a mudança dos termos melhor ranqueados foi significativa ao longo da construção do modelo. Algumas observações interessantes: (i) os termos mais frequentes no primeiro momento foram, em sua totalidade, diferentes dos termos no momento 1.700; (ii) o termo washington fez parte do ranking no intervalo de 100 até 500 documentos, sendo que aparece novamente no momento 900 e, novamente, na faixa de 


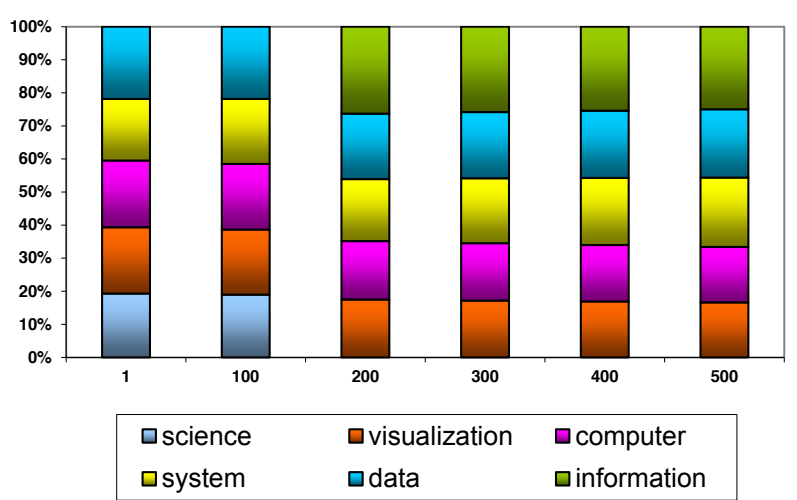

Figura 6.3: Ranking dos cinco termos mais frequentes no modelo vetorial incremental, para o conjunto CBR-ILP-IR, em diferentes instantes de tempo.

1.300 até 1.700 . Isso evidencia como o modelo vetorial incremental adapta-se à natureza da coleção, conforme novos documentos são adicionados.

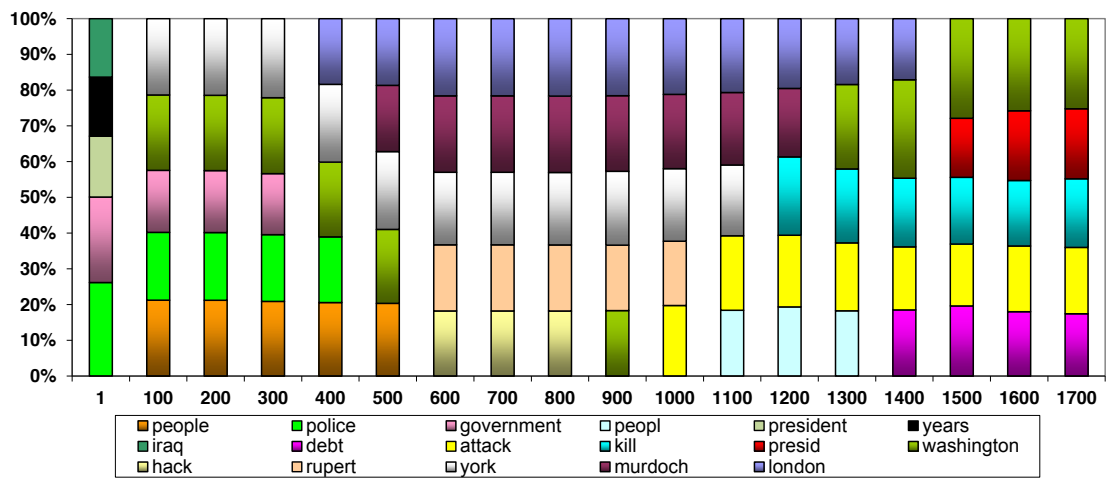

Figura 6.4: Ranking das cinco primeiras palavras com maior $D F$ no modelo vetorial incremental, para conjunto de textos News2011, para diferentes instantes de tempo.

Em seguida, mapas visuais foram gerados utilizando o iVSM e VSM, que foram posteriormente comparados segundo seus valores de precisão NH e Coeficiente de Silhueta (CS). Não consideramos o CS de layouts gerados por projeções LSP porque o cálculo das distâncias no espaço bidimensional é sensível ao formato do agrupamento, e tende a privilegiar agrupamentos de formatos arredondados. Como tal, não é necessariamente uma medida significativa da qualidade de agrupamentos no espaço visual quando os formatos dos grupos variam amplamente. Os conjuntos de textos CBR-ILP-IR e News2011 foram empregados, e duas estratégias de pré-processamento clássicas utilizadas: remoção de stopwords e redução dos termos a seus radicais. Os valores dos cortes de Luhn para o iVSM são calculados automaticamente, tal como definido nas Equações 3.5 e $3.6 \mathrm{com}$ cli $=2 \%$ e $c l s=90 \%$, enquanto para o VSM foi aplicado corte inferior igual a dez, e nenhum corte 
superior. Em ambos os casos, a matriz de dissimilaridade foi calculada usando a medida do cosseno.

As curvas NH das visualizações construídas com as técnicas LSP e NJ, para o conjunto CBR-ILP-IR, são apresentadas nas Figuras 6.5 e 6.7, em que as linhas de cor azul representam os mapas visuais gerados com a técnica iVSM e as de cor vermelha as geradas com a VSM. A Figura 6.5 apresenta os resultados de precisão para as projeções LSP (pontos de controle $=57$ e número de vizinhos $=15$ ). No gráfico observa-se que os valores de precisão das duas técnicas encontram-se próximos, com valores entre 0,9 e 0,96. Isso indica que ambas geraram layouts nos quais são facilmente identificáveis as três classes do conjunto de textos. Nota-se, também, que o iVSM apresentou melhores resultados, comparado ao VSM. Com o intuito de inferir se essa diferença é estatisticamente significativa utilizamos o teste de hipóteses denominado "Teste t de Student". O Teste t comprova se a média de dois grupos é significativamente diferente. Formulamos a hipótese nula ("As duas curvas não tem diferença significativa") e, consequentemente, a hipótese alternativa ("As duas curvas tem diferença significativa"). Nos experimentos, o nível de significância foi definido em $\alpha=0,05$. A variável p-valor é utilizada para sintetizar o resultado do teste. A hipótese nula pode ser rejeitada caso o p-valor seja menor do que $\alpha$.

O p-valor obtido para as curvas NH da Figura 6.5 foi 1,629E-11 (p-valor menor do que $0,05)$ indicando que a diferença entre os valores de precisão das duas curvas é estatisticamente significativo.

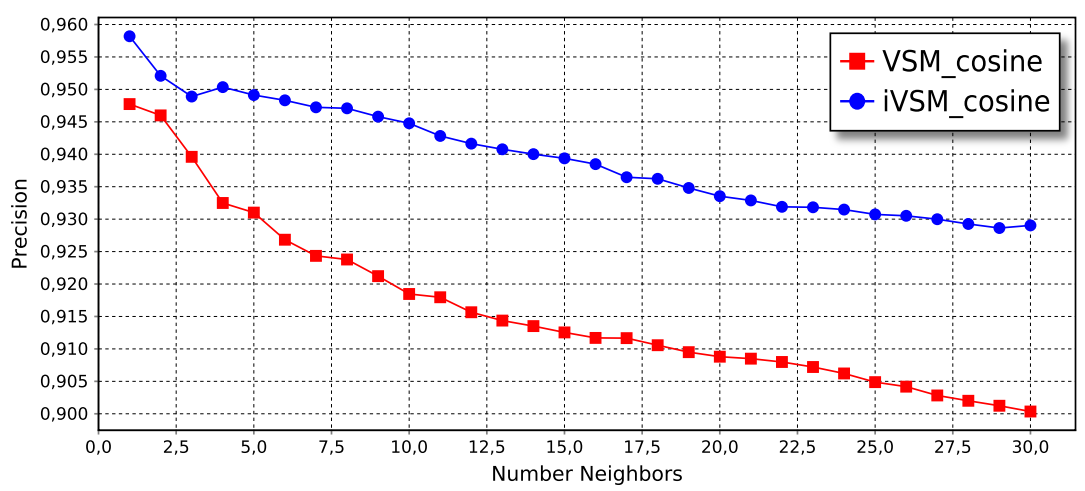

Figura 6.5: Resultados de precisão das projeções LSP geradas para o conjunto do artigos CBR-ILP-IR.

Os layouts gerados para o conjunto de dados CBR-ILP-IR usando a técnica LSP são mostrados na Figura 6.6, em que os documentos são representados como círculos e coloridos de acordo com sua classe. As projeções revelam as três classes existentes no conjunto de dados, sendo que os círculos de cor vermelha são documentos que pertencem 
à classe IR, os de cor verde são da classe ILP e finalmente a classe CBR é representada pela cor azul. Ambas as projeções apresentam alguma mistura entre classes, mas algumas diferenças são claramente identificáveis. Por exemplo, a projeção gerada com o VSM (ver Figura 6.6(a)) apresenta alguns elementos da classe vermelha junto aos da classe azul (círculo laranja) o que acontece em menor quantidade na projeção gerada com o iVSM. Por outro lado, misturas entre as classes verde e vermelha (círculo azul claro) aparecem em ambas as projeções, isso pode se dever ao fato de que a classificação desse conjunto é uma rotulação baseada na fonte do artigo, e não uma classificação feita com base no conteúdo dos textos. Observa-se, também, que os elementos das três classes ficaram mais próximos entre si na Figura 6.6(b), o que permite identificar claramente os três grupos, mas isso também provoca maior sobreposição de pontos que na projeção gerada com o modelo vetorial, o que poderia tornar difícil uma análise local na classe.

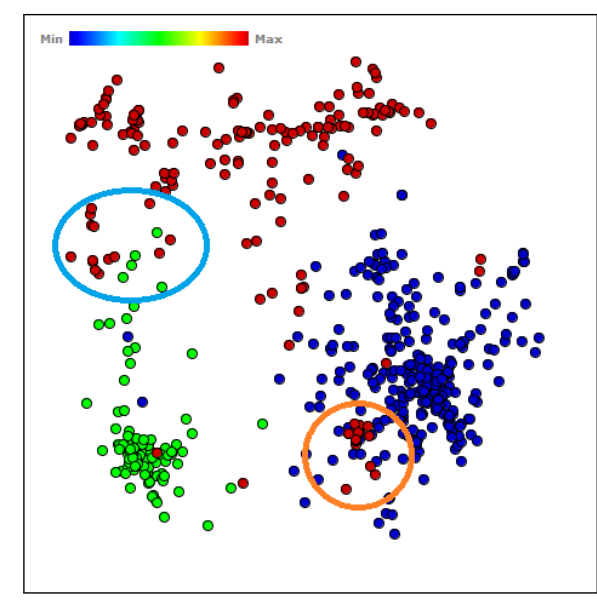

(a) VSM

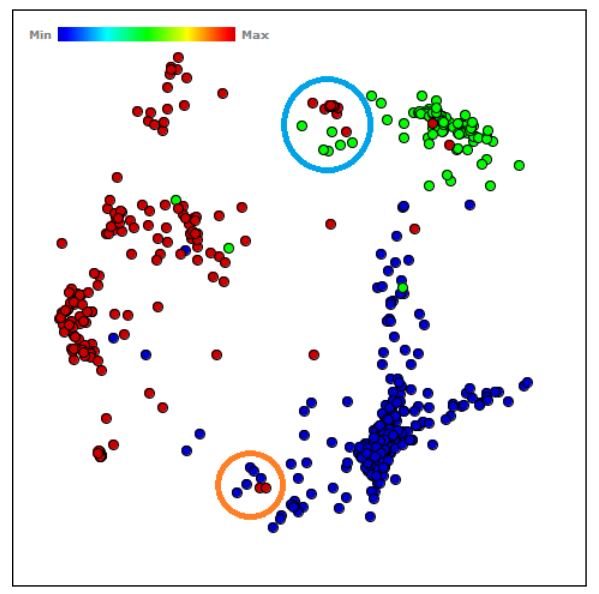

(b) iVSM

Figura 6.6: Projeções multidimensionais para o conjunto de dados CBR-ILP-IR. Ambas as projeções apresentam misturas entre as classes existentes no conjunto de dados, por exemplo, os elementos indicados no interior dos circulo azul claro e laranja. Elementos das três classes ficaram mais próximos entre si no layout gerado com o modelo iVSM, o que permite identificar claramente os três grupos, mas isso também provoca maior sobreposição de pontos que na projeção gerada com o modelo vetorial, o que poderia tornar difícil uma análise local na classe.

A Figura 6.7 expõe os resultados de precisão para as árvores NJ desse mesmo conjunto de textos. Novamente a técnica iVSM apresentou melhores resultados, comparado ao VSM. O p-valor obtido para esse gráfico foi muito próximo de zero (p-valor=1,199E-14), pelo que a diferença entre estas curvas é estatisticamente significativa. Na Figura 6.8 são apresentadas as árvores NJ geradas para este conjunto de textos. Análogo às projeções LSP, as duas árvores refletem as três classes do conjunto de dados. Ambas as árvores 
apresentam alguma mistura entre as três classes, mas o layout gerado com o modelo incremental as apresenta em menor quantidade. Por exemplo, mistura de elementos das classes vermelha e verde dentro da circunferência laranja, é mais acentuada no modelo vetorial VSM. O mesmo acontece com os elementos dentro das circunferências verde e azul claro, pelo que se conclui que a árvore gerada com o iVSM agrupou melhor, visualmente, os elementos. O coeficiente de silhueta reforça esta percepção. O CS da árvore gerada com o iVSM é igual a 0,1450, contra o valor 0,0406 para o VSM. A análise numérica dos resultados (NH e CS) indica que, para o conjunto de textos CBR-ILP-IR, o modelo vetorial incremental obteve melhores resultados, comparado ao VSM, o que coincide com a inspeção visual.

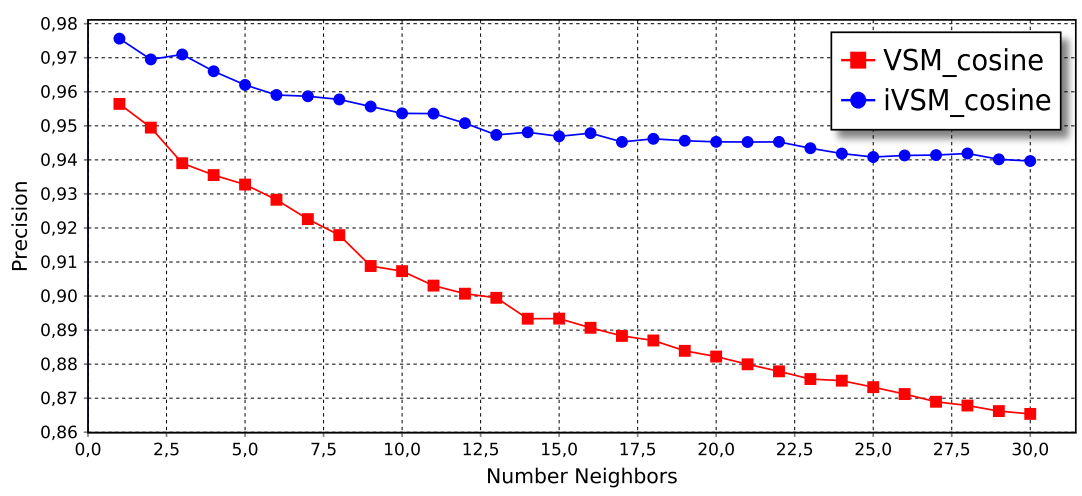

Figura 6.7: Resultados de precisão das árvores NJ geradas para o conjunto do artigos CBR-ILP-IR, utilizando a medida do cosseno sobre os modelos VSM e iVSM.

A seguir são apresentadas as curvas de precisão e mapas visuais correspondentes ao conjunto de notícias News2011. Na Figura 6.9 são apresentadas as curvas NH correspondentes às projeções geradas com a técnica LSP utilizando 177 pontos de controle e 15 vizinhos. Nota-se que, contrário aos resultados obtidos para o conjunto de artigos científicos, a projeção gerada como o VSM apresentou melhores resultados que o iVSM, com valores entre 0,85 e 0,945. Utilizando novamente o teste t de Student, verificamos que esta diferença é considerada estatisticamente significativa ( $\mathrm{p}$-valor=0,029). Uma análise visual é feita para verificar se estas diferenças são evidenciadas nos layouts gerados, os quais são apresentados na Figura 6.10. Embora alguma mistura entre grupos seja inevitável, devido ao número de classes na coleção (23 classes), algumas diferenças são claramente identificáveis. Por exemplo, elementos da classe amarela (circunferência preta) ficaram mais bem agrupados no layout gerado com o VSM. No layout gerado pelo iVSM, os elementos misturados dentro da circunferência rosa pertencem a três classes diferentes, que foram separadas na projeção a esquerda. Encontramos outros dois casos similares, 


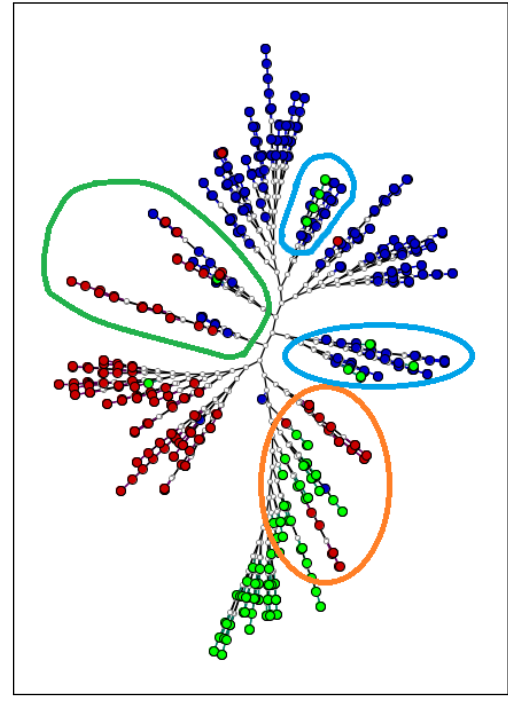

(a) VSM

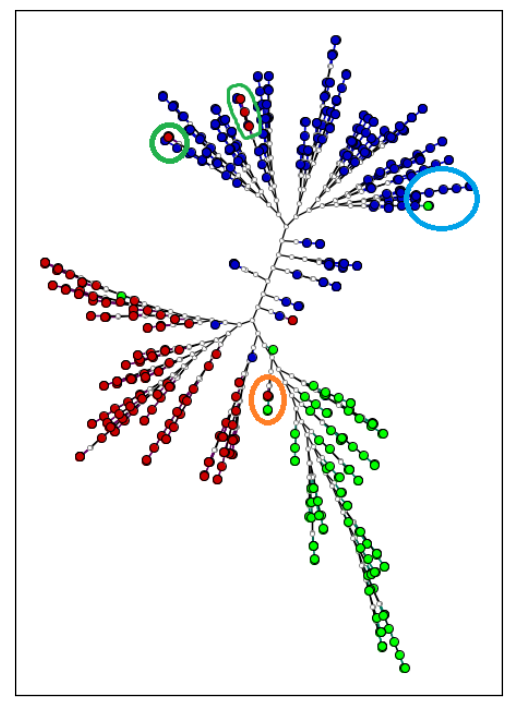

(b) iVSM (gráfico rotacionado $\left.90^{\circ}\right)$

Figura 6.8: As árvores NJ geradas para o conjunto de textos CBR-ILP-IR. As duas árvores refletem as três classes do conjunto de dados, mas com alguma mistura entre as classes. O layout gerado com o modelo incremental apresentou menos mistura entre elementos de diferentes classes, como se observa nos setores indicados pelas circunferências nas cores verde, laranja e azul claro.

com os elementos dentro das circunferências roxa e cinza, que novamente ficaram mais bem agrupados na projeção gerada com o VSM. Obtivemos casos também favoráveis para a projeção da direita, por exemplo, os elementos da classe laranja (circunferência azul claro), que ficaram mais bem agrupados comparados à projeção do modelo vetorial. Ainda assim, de modo geral os grupos são mais bem identificados na projeção gerada com o VSM.

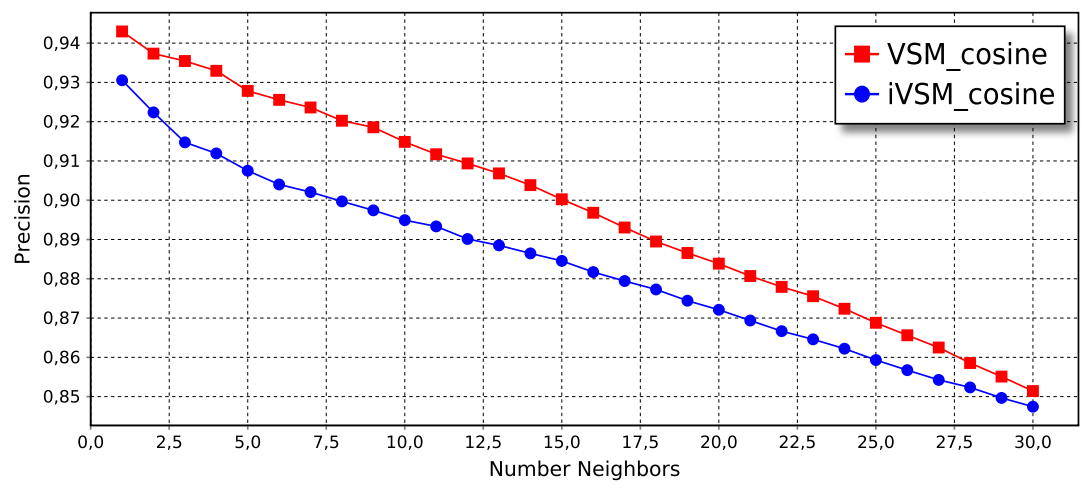

Figura 6.9: Resultados de precisão das projeções LSP geradas para o conjunto News2011. 


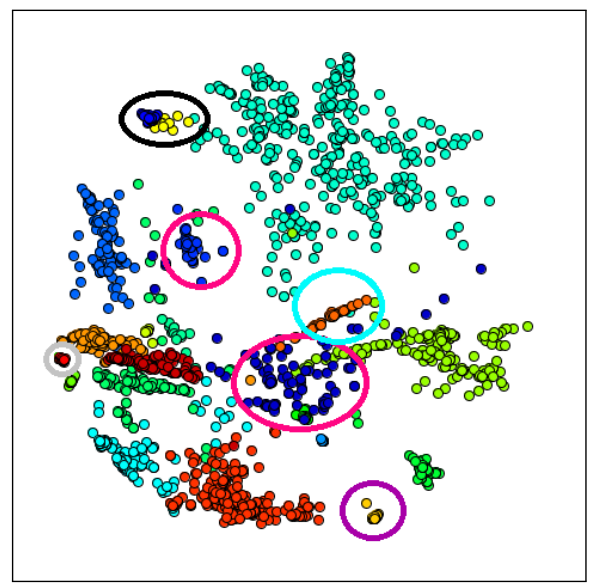

(a) VSM

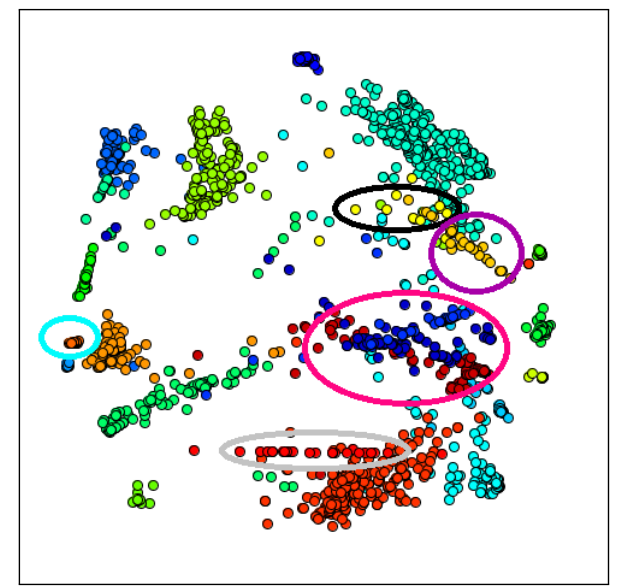

(b) iVSM

Figura 6.10: Projeções multidimensionais LSP para o conjunto de textos News2011. Embora alguma mistura entre grupos seja inevitável, devido ao número de classes na coleção (23 classes), algumas diferenças são identificáveis. Por exemplo, elementos da classe amarela (circunferência preta) ficaram mais bem agrupados no layout gerado com o VSM do que no layout gerado com o iVSM. O mesmo acontece com os elementos das circunferências rosa, roxa e cinza.

Já na Figura 6.11 são apresentadas as curvas de precisão dos mapas visuais gerados com a NJ. Aqui, ambas as curvas são muito próximas, com valores entre 0,87 e 0,97. Esses valores de precisão obtidos com a árvore NJ são melhores que os da projeção LSP, pelo que a árvore identificou e agrupou melhor as classes da coleção. Nota-se que a curva gerada com o modelo vetorial incremental é ligeiramente melhor do que a do VSM. Realizado o teste estatístico, descobrimos que esta diferença não é significativa, pois o p-valor obtido foi 2,256 (p-valor > 0,05). As árvores NJ da coleção são exibidas na Figura 6.12. Realizar um análise visual torna-se difícil devido à quantidade de elementos, classes e cores presentes no layout. A ferramenta Vispipeline oferece um módulo (Identity Coordination) que ajuda na exploração do mapa, permitindo-nos identificar e localizar a presença de um mesmo elemento presente em dois mapas distintos. Elementos selecionados mantem a sua cor e aplica-se transparência aos não selecionados. Alguns exemplos são apresentados na Figura 6.13. Nas Figuras 6.13(a) e 6.13(b) elementos da ciano foram selecionados, observamos que enquanto o VSM agrupou todos os elementos em um só ramo, o iVSM separou a classe em dois diferentes ramos. Ambas as árvores apresentaram alguns elementos da classe verde claro, adicionalmente o layout da esquerda apresentou também elementos das classes azul e vermelho claro. Nas Figuras 6.13(c) e 6.13(d) elementos da classe verde limão foram selecionados. Novamente o layout gerado pelo modelo vetorial incremental 
dividiu a classe em dois ramos distintos. Ambas as seleções apresentaram um elemento da classe vermelho claro. Por último, nas Figuras 6.13(e) e 6.13(f) foram selecionados elementos da classe verde claro. Para esta classe, o layout gerado com o iVSM agrupou melhor os elementos em comparação ao gerado com o VSM, no qual misturou-se com elementos das classes azul, laranja e verde escuro. Em termos do CS, o valor obtido pelo modelo VSM $(\mathrm{CS}=0,2891)$ foi apenas ligeiramente superior ao iVSM (CS=0,2583), indicando que as duas técnicas são, para este conjunto de textos, quantitativamente similares, o que coincide com a inspeção visual.

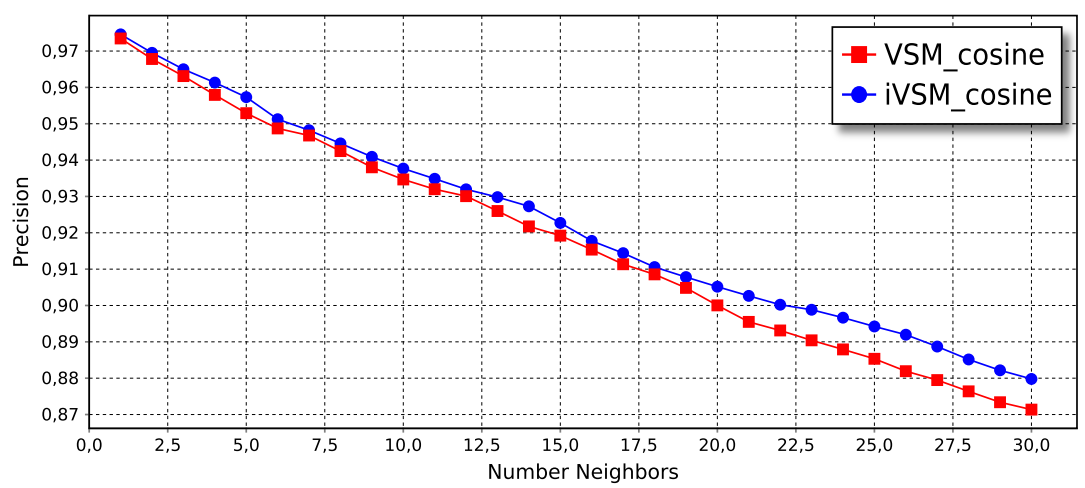

Figura 6.11: Resultados de precisão das árvore NJ geradas para o conjunto News2011.

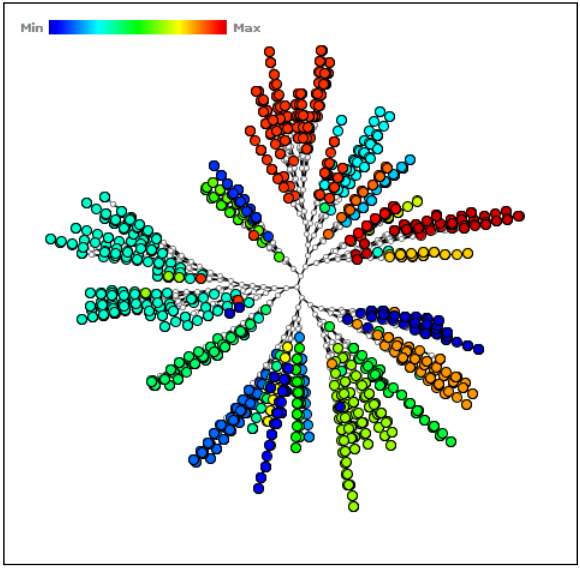

(a) VSM

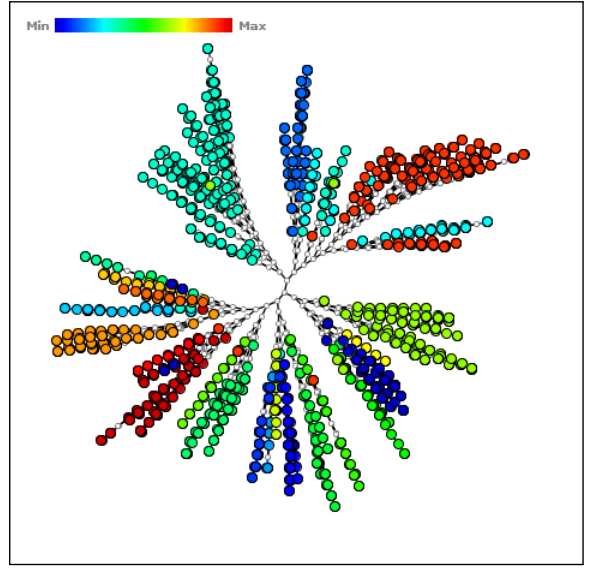

(b) iVSM

Figura 6.12: Árvores NJ geradas para o conjunto de textos News2011. Realizar um análise visual torna-se difícil devido à quantidade de elementos, classes e cores presentes no layout. Na Figura 6.13 uma exploração dessas árvores é realizado, utilizando o módulo Identity Coordination da ferramenta Vispipeline. 


\subsection{Experimento 3: Comparando Medidas sobre Strings e sobre Modelos Vetoriais}

Neste experimento comparamos a capacidade de preservação de vizinhança de layouts obtidos utilizando matrizes de distância calculados com medidas de similaridade entre strings- as analisadas anteriormente, mais a NCDs, além da similaridade do cosseno calculada sobre os modelos vetoriais VSM e iVSM, para os três conjuntos de dados. O pré-processamento aplicado aos textos varia dependendo da medida de dissimilaridade empregada. Para os três conjuntos de dados, na geração dos modelos VSM e iVSM foi aplicada a remoção de stopwords (geral e de domínio específico). Para o VSM adota-se um limiar de Luhn inferior igual a 10 e para iVSM os limiares foram obtidos automaticamente, conforme as Equações 3.5 e $3.6 \mathrm{com} c l i=2 \%$ e $\mathrm{cls}=90 \%$. Para as matrizes de distância baseadas em métricas de comparação de strings utilizamos as configurações estabelecidas na Tabela 5.4.

Os resultados de precisão obtidos para o conjunto CBR-ILP-IR são apresentados na Figura 6.14. Foram consideradas duas configurações na LSP: com 57 e 177 pontos de controle, ambos com 15 vizinhos mais próximos. Na Figura 6.14(a) são apresentados os resultados do NH para a primeira configuração. Observamos que as projeções com maior precisão foram as geradas com matrizes de dissimilaridade obtidas por a distância do cosseno sobre o modelo iVSM, o Coeficiente de Dice e a medida do cosseno_S, com valores entre 0,9 e 0,97. Observamos, também, que as estratégias de pré-processamento aplicadas previamente ao cálculo das dissimilaridades, afetaram consideravelmente os resultados gerados pelo modelo vetorial, comparados aos da Figura 6.5. Ao contrário do VSM, o modelo iVSM não foi afetado por esta mudança no pré-processamento. As projeções identificadas como as menos precisas foram as geradas com matrizes de dissimilaridade computadas com as métricas NCDs e Coeficiente de Matching. Os resultados de precisão para a segunda configuração utilizada, com 177 pontos de controle, são ilustrados na Figura 6.14(b). As métricas com melhores resultados foram o Coeficiente de Dice, Coeficiente de Overlap e a similaridade do cosseno_S, que praticamente apresentaram a mesma precisão. Note-se que projeções LSP geradas com a primeira configuração resultaram mais precisas do que as geradas em esta segunda configuração. Por último, na Figura 6.14(c) são apresentadas as curvas de precisão geradas para as árvores NJ. Aqui, os melhores resultados foram obtidos por quatro métricas de comparação de strings: Coeficiente de Overlap, Qgram, NCDs e cosseno_S, que ficaram muito próximas com valores entre 0,95 e 0,98. É interessante notar que, além da métrica Coeficientes de Matching, a árvore gerada com o Vector 
Space Model (VSM) convencional ficou como uma das menos precisas. Logo, os CS foram calculados, sendo que as árvores que separaram melhor as agrupações foram as matrizes geradas com Coeficientes de Overlap ( $\mathrm{CS}=0,3668)$ e o modelo iVSM ( $\mathrm{CS}=0,3631)$.

Para o conjunto de textos News2011, as projeções LSP são geradas com duas novas configurações. A primeira delas considera 150 pontos de controle e 20 vizinhos mais próximos. Os resultados de precisão NH para esta configuração são apresentados na Figura 6.15(a). Observamos que os modelos vetoriais, VSM e iVSM, obtiveram melhores resultados que as métricas de comparação de strings, sendo que as que apresentaram os piores resultados para esta configuração, foram a NCDs e o similaridade do cosseno_S. Na segunda configuração LSP, ilustrada na Figura 6.15(b), utilizam-se 177 pontos de controle e 15 vizinhos mais próximos. Aqui, o iVSM mantem-se como a mais precisa, seguida pela VSM e a métrica Qgram. Na Figura 6.15(c), apresentamos os gráficos NH para as árvores NJ. As árvores construídas com matrizes de dissimilaridade calculadas com o modelo de espaço vetorial convencional, o iVSM e a métrica Qgram obtiveram os melhores resultados, praticamente apresentaram a mesma precisão, com valores entre 0,875 e 0,975 seguidas de perto pelas métricas cosseno_S, Coeficiente de Dice e Coeficiente de Overlap. O CS de tais árvores revelam que o modelo iVSM $(\mathrm{CS}=0,2762)$ apresentou melhor coesão e separação entre os grupos definidos pelas classes, seguido pelo VSM $(\mathrm{CS}=0,2495)$ e a métrica Qgram $(\mathrm{CS}=0,2028)$.

Por último, na Figura 6.16 são apresentados os gráficos NH para o conjunto de notícias NewsReuters utilizando a técnica de projeção LSP. Duas configurações para as projeções LSP são utilizadas: (i) a primeira com 200 pontos de controle e 20 vizinhos mais próximos e, (ii) uma segunda com 398 pontos de controle e 15 vizinhos mais próximos. Os gráficos de precisão para estas duas configurações são ilustrados nas Figuras 6.16(a) e 6.16(b), respectivamente. Observa-se que em ambos os gráficos, as matrizes construídas com o modelo iVSM obtiveram os melhores resultados de precisão, seguido pelo VSM e a similaridade do cosseno_S, sendo que para estes últimos os valores de precisão foram melhores na primeira configuração utilizada. Os melhores resultados de precisão NH para layouts gerados com NJ (Figura 6.16(c)) foram obtidos por a medidas baseadas na comparação de strings cosseno_S e Coeficiente de Dice, ademais da distância do cosseno aplicada ao modelo iVSM. Layouts computados como a NCDs e a Qgram mostraram os piores resultados. Apesar do seu desempenho inferior, essas medidas produziram curvas com valores acima de 0,87 .

A Tabela 6.1 apresenta um ranking aproximado das posições das curvas NH geradas com as medidas de similaridade computadas nesse experimento. 


\begin{tabular}{|c|c|c|c|c|c|}
\hline \multirow[b]{2}{*}{ Corpus } & \multirow[b]{2}{*}{ Técnica } & \multicolumn{4}{|c|}{ Ranking } \\
\hline & & $1^{\circ}$ & $2^{\circ}$ & $3^{\circ}$ & $4^{\circ}$ \\
\hline \multirow{3}{*}{ CBR-ILP-IR } & LSP (1) & iVSM & Coef. Dice & cosseno_S & Qgram \\
\hline & LSP (2) & Coef. Dice & cosseno_S & Coef. Overlap & iVSM \\
\hline & NJ & Coef. Overlap & Qgram & NCDs & cosseno_S \\
\hline \multirow{3}{*}{ News2011 } & LSP (1) & iVSM & VSM & Qgram & Coef. Overlap \\
\hline & LSP (2) & iVSM & VSM & Qgram & Coef. Overlap \\
\hline & NJ & iVSM & VSM & Qgram & Coef. Dice \\
\hline \multirow{3}{*}{ NewsReuters } & LSP (1) & iVSM & VSM & cosseno_S & Coef. Dice \\
\hline & $\operatorname{LSP}(2)$ & iVSM & VSM & cosseno_S & Coef. Dice \\
\hline & NJ & cosseno_S & Coef. Dice & iVSM & Coef. Overlap \\
\hline \multirow[b]{2}{*}{ Corpus } & & \multicolumn{4}{|c|}{ Ranking } \\
\hline & Técnica & $5^{\circ}$ & $6^{\circ}$ & $7^{\circ}$ & $8^{\circ}$ \\
\hline \multirow{3}{*}{ CBR-ILP-IR } & LSP (1) & VSM & Coef. Overlap & Coef. Matching & NCDs \\
\hline & LSP (2) & VSM & NCDs & Coef. Matching & Qgram \\
\hline & NJ & iVSM & Coef. Dice & VSM & Coef. Matching \\
\hline \multirow{3}{*}{ News2011 } & LSP (1) & cosseno_S & NCDs & Coef. Matching & Coef. Dice \\
\hline & LSP (2) & Coef. Matching & Coef. Dice & cosseno_S & NCDs \\
\hline & NJ & cosseno_S & Coef. Overlap & Coef. Matching & NCDs \\
\hline \multirow{3}{*}{ NewsReuters } & LSP (1) & Coef. Overlap & Qgram & NCDs & Coef. Matching \\
\hline & LSP (2) & Coef. Overlap & Qgram & Coef. Matching & NCDs \\
\hline & NJ & VSM & Coef. Matching & Qgram & NCDs \\
\hline
\end{tabular}

Tabela 6.1: Ranking das curvas NH dos layouts obtidos com métricas baseadas em strings e a distância do cosseno computada sobre os modelos VSM e iVSM, para os três conjuntos de textos.

\subsection{Considerações Finais}

Este capítulo apresentou um estudo comparativo das diferentes medidas de similaridade de texto e como elas afetam a qualidade de mapas visuais baseados no posicionamento de pontos. Concentramo-nos, principalmente, em duas abordagens: as baseadas na representação vetorial (VSM e iVSM) e em medidas de comparação de strings.

$\mathrm{Na}$ Seção 6.2, resultados de precisão $\mathrm{NH}$ obtidos de layouts gerados com matrizes de dissimilaridade computadas com quinze diferentes medidas baseadas em strings, foram comparados. Cinco medidas que apresentaram melhor desempenho foram: Qgram, Cosseno, Coeficiente de Dice, Coeficiente de Overlap e o Coeficiente de Matching. Na Seção 6.3 apresentou-se uma avaliação qualitativa e quantitativa dos layouts obtidos matrizes de dissimilaridade computadas empregando a distância do cosseno sobre as representações VSM convencional e iVSM, respectivamente.

Já na Seção 6.4, layouts gerados com matrizes de dissimilaridade computadas com as abordagens foram comparados. Descobrimos que ambas as abordagens têm pontos a favor, mas de forma geral, o iVSM caracterizou-se por apresentar os melhores resultados quantitativos em muitas das configurações testadas, o que coincidiu com a inspeção visual. 
Finalmente, os resultados mostraram que uma representação adequada do conjunto de textos é de suma importância para o cálculo de similaridade e, por consequência, importante para a qualidade da visualização resultante. 


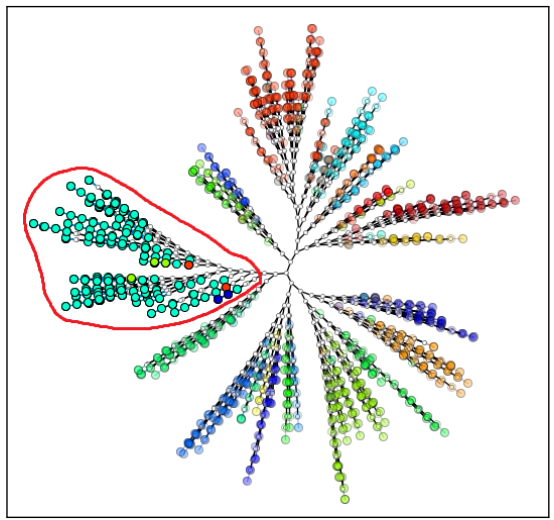

(a) VSM

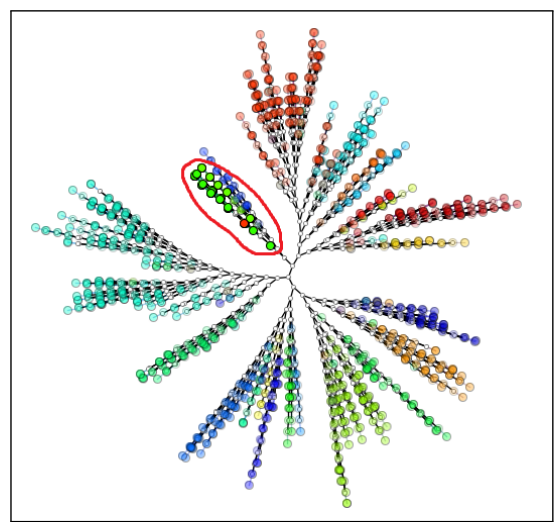

(c) VSM

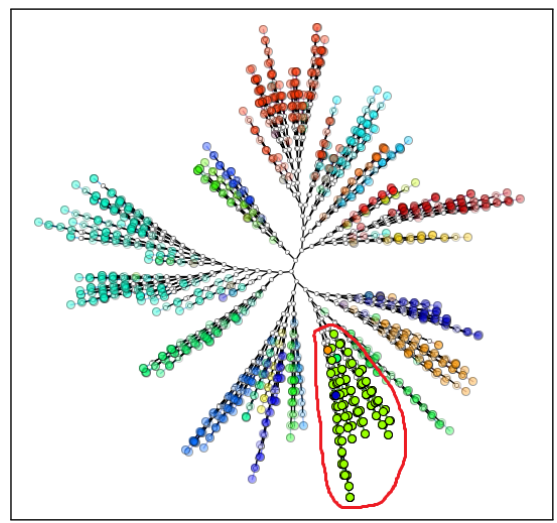

(e) VSM

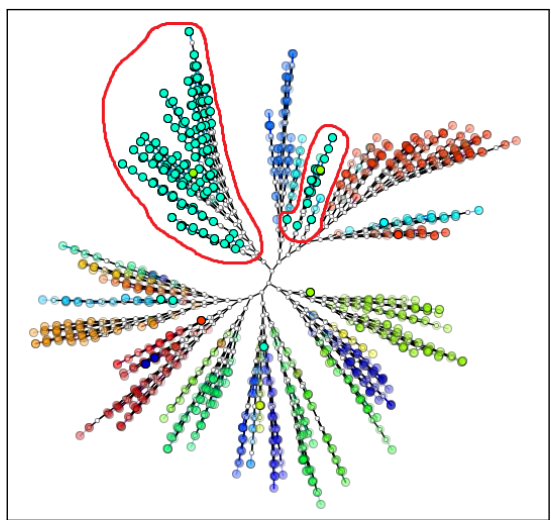

(b) iVSM

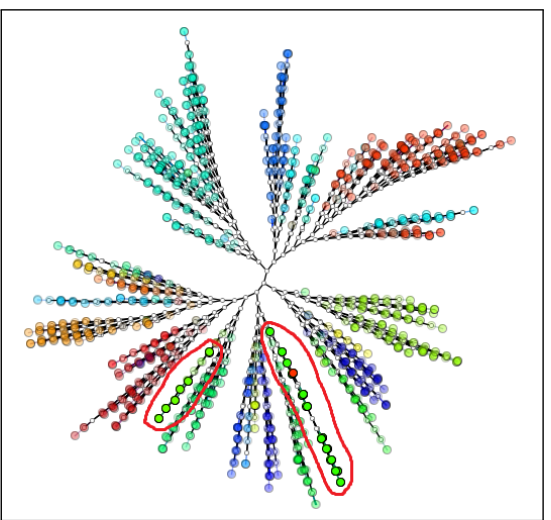

(d) iVSM

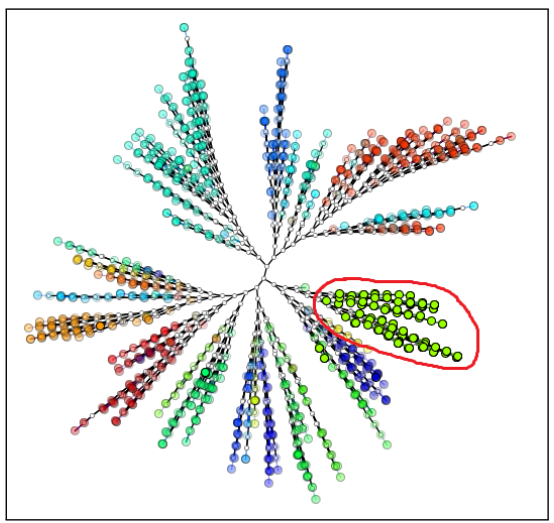

(f) iVSM

Figura 6.13: Exploração das árvores NJ do conjunto News2011 utilizando o módulo Identity Coordination. Nas Figuras 6.13(a) e 6.13(b) elementos da cor ciano foram selecionados, observa-se que enquanto o VSM agrupou todos os elementos em um só ramo, o iVSM separou a classe em dois diferentes ramos. Nas Figuras 6.13(c) e 6.13(d) elementos da classe verde limão foram selecionados. Novamente o layout gerado pelo modelo vetorial incremental dividiu a classe em dois ramos distintos. Por último, nas Figuras 6.13(e) e 6.13(f) foram selecionados elementos da classe verde claro. Para esta classe, o layout gerado com o iVSM agrupou melhor os elementos em comparação ao gerado com o VSM, no qual misturou-se com elementos das classes azul, laranja e verde escuro. 


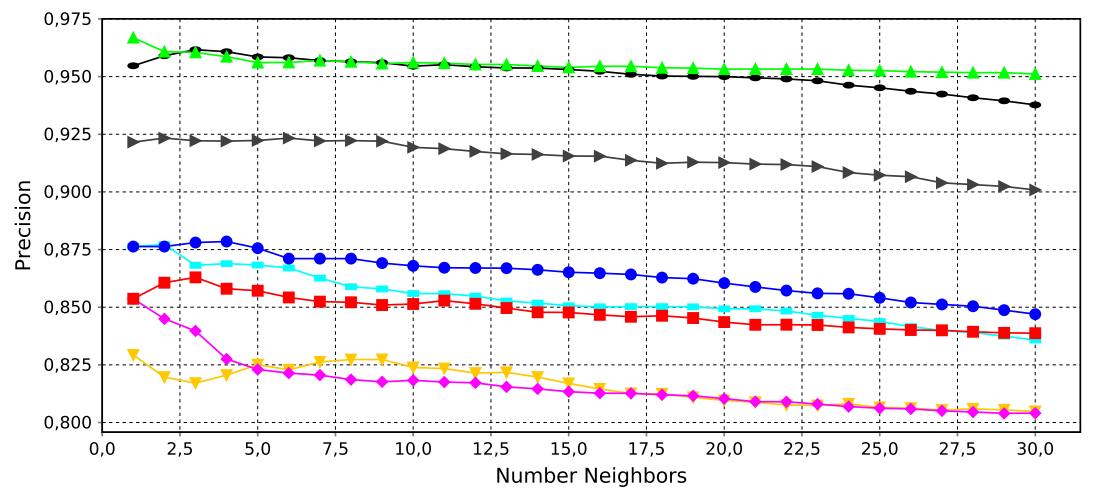

- Overlap's C $\bullet$ qgram $₫$ iVSM_cosine $\rightarrow$ ncds - VSM_cosine $\rightarrow$ Matching's C $\rightarrow$ Dice's $C \rightarrow$ cosine

(a) LSP pontos de controle:57 vizinhos:15

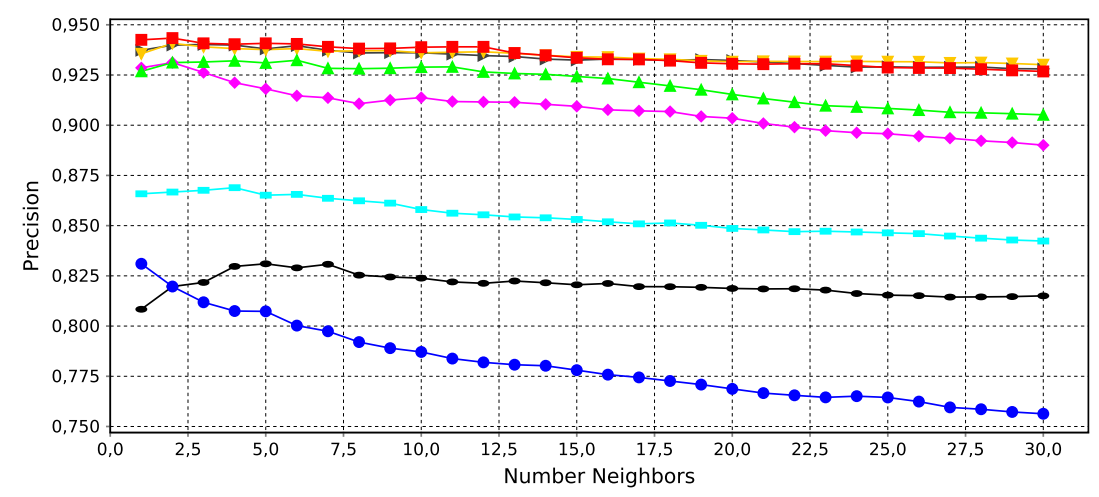

- Overlap's C $\bullet$ qgram $\rightarrow$ iVSM_cosine $\rightarrow$ VSM_cosine - ncds $\rightarrow$ Dice's C $\rightarrow$ Matching's $C \rightarrow$ cosine

(b) LSP pontos de controle:177 vizinhos:15

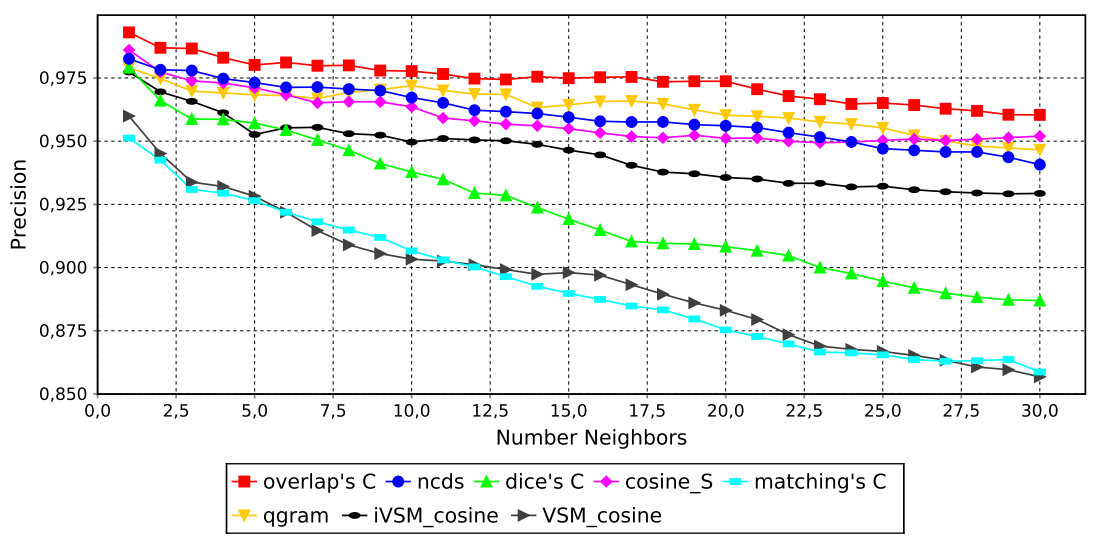

(c) NJ

Figura 6.14: Resultados de precisão das projeções geradas para o conjunto CBR-ILP-IR 


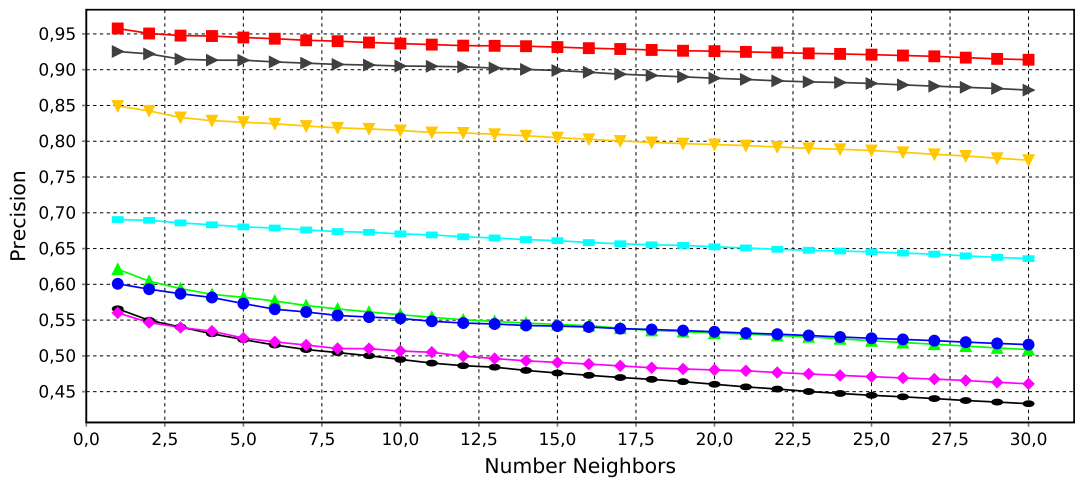

$\rightarrow$ iVSM_cosine $\rightarrow$ Matching's C $\rightarrow$ Dice's $C \rightarrow$ cosine - Overlap's C $\forall$ qgram $\rightarrow$ ncds $\rightarrow$ VSM_cosine

(a) LSP pontos de controle:150 vizinhos:20

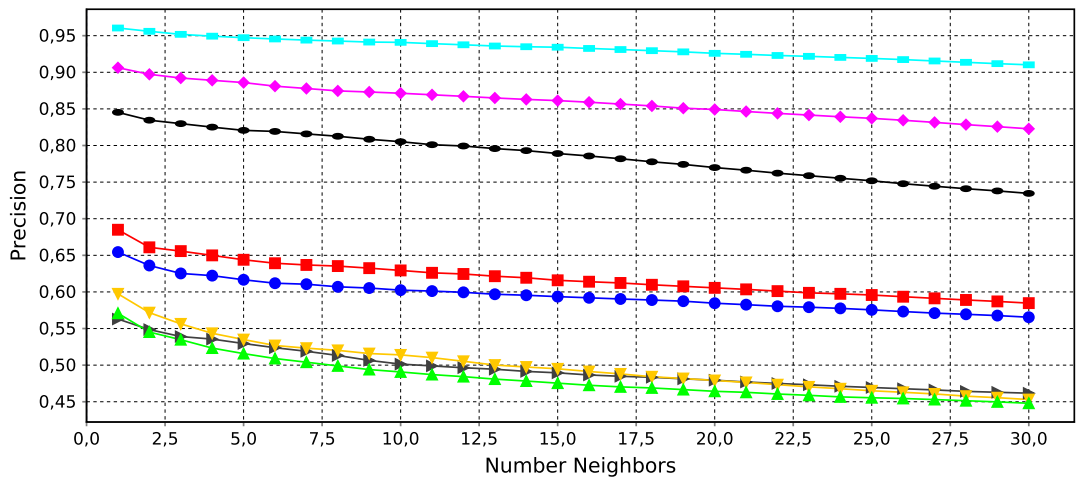

- Overlap's C $\rightarrow$ cosine $₫$ Dice's C $\bullet$ VSM_cosine - iVSM_cosine $\rightarrow$ ncds $\rightarrow$ qgram $\rightarrow$ Matching's C

(b) LSP pontos de controle:177 vizinhos:15

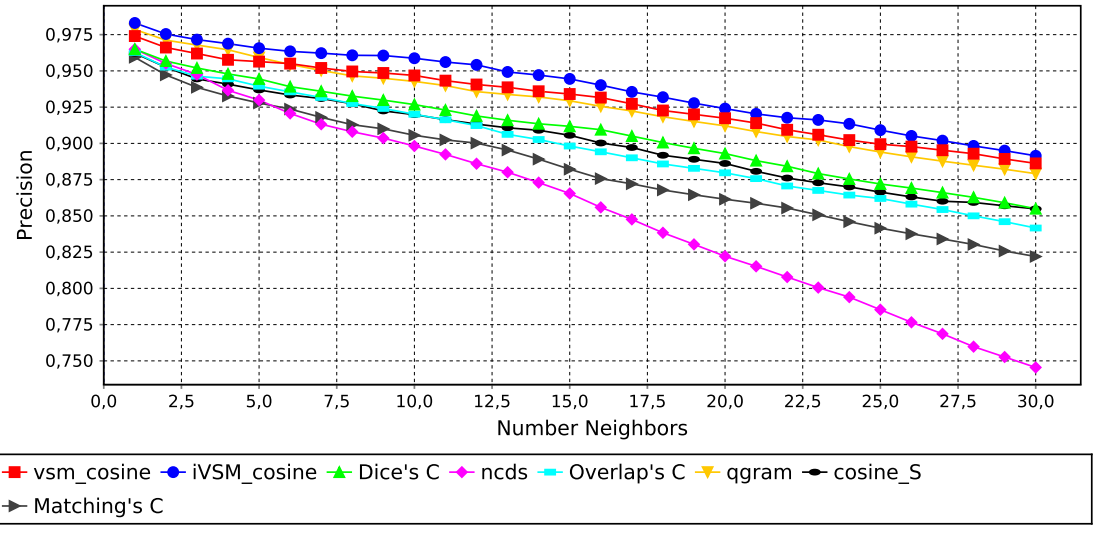

(c) NJ

Figura 6.15: Resultados de precisão das projeções geradas para o conjunto News2011 


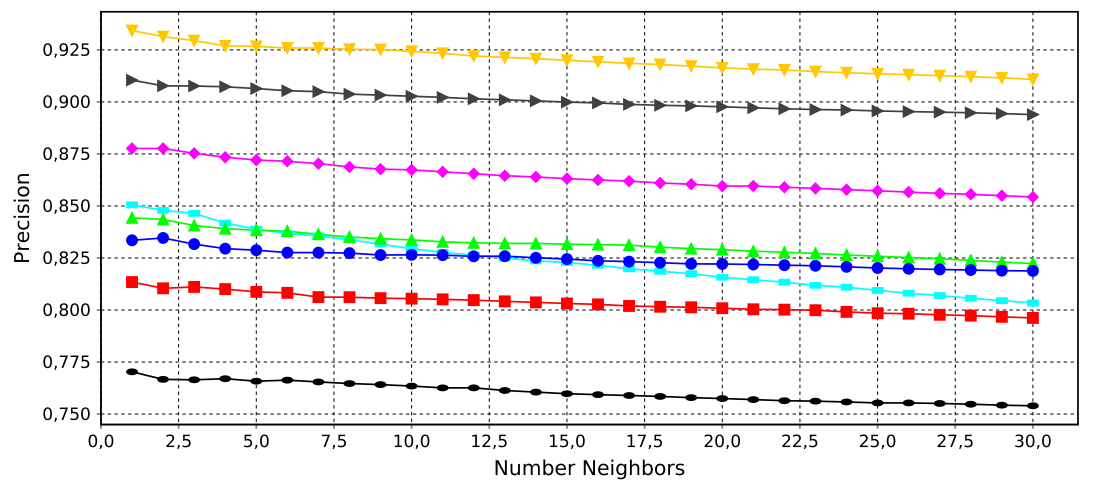

$\rightarrow$ Matching's C $\rightarrow$ Overlap's C $\rightarrow$ Dice's C $\rightarrow$ cosine - - qgram $\rightarrow$ iVSM_cosine $\rightarrow$ ncds $\rightarrow$ VSM_cosine

(a) LSP pontos de controle:200 vizinhos:20

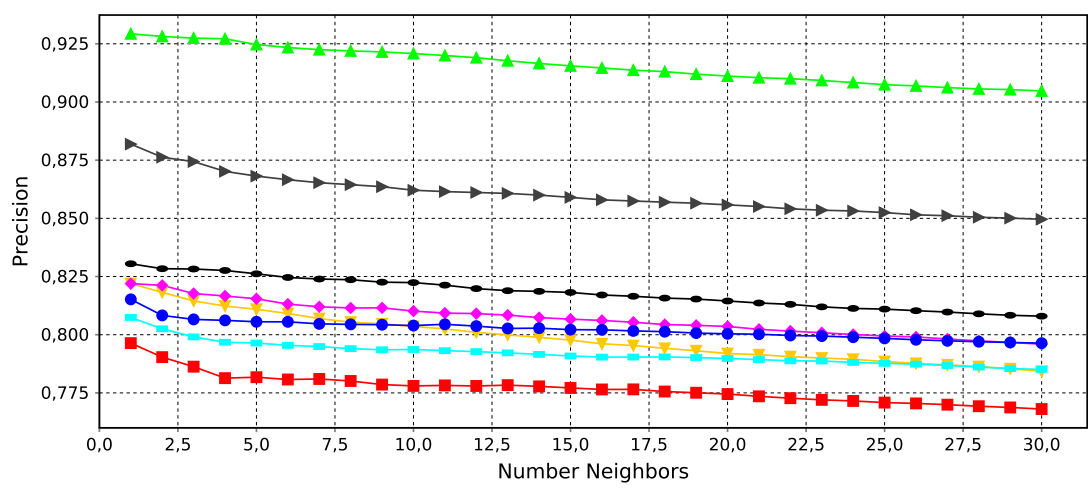

- Matching's C $\bullet$ Overlap's C $\rightarrow$ iVSM_cosine $\rightarrow$ Dice's C - ncds $\rightarrow$ qgram $\rightarrow$ cosine $\rightarrow$ VSM_cosine

(b) LSP pontos de controle:398 vizinhos:15

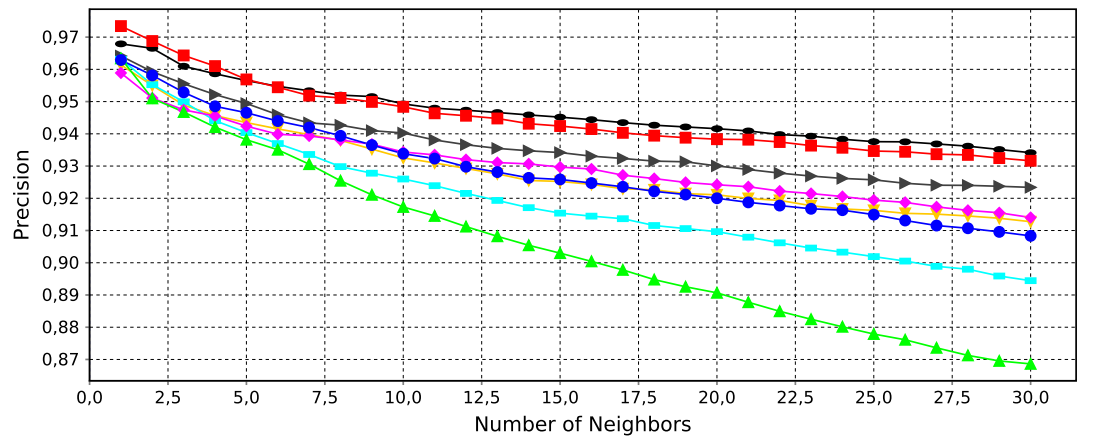

- Dice's C $\bullet-$ Matching's C $\rightarrow$ NCDs $\bullet$ Overlap's C - Qgram $\rightarrow$ VSM_cosine $\bullet$ cosine_S $\rightarrow$ iVSM_cosine

(c) NJ

Figura 6.16: Resultados de precisão das projeções geradas para o conjunto NewsReuters 


\section{Conclusões}

Nesta dissertação foi apresentado um estudo de como o pré-processamento do texto, com especial atenção à eleição de uma medida de (dis)similaridade, afeta a qualidade dos layouts produzido por técnicas de visualização de documentos, em termos de agrupamento e separação de itens com conteúdo altamente relacionado.

Duas abordagens foram consideradas: as medidas de similaridade aplicadas às representações vetoriais dos documentos; e medidas de distância entre textos baseadas na comparação de strings. Para ambas as abordagens, mapas visuais baseados em similaridade foram obtidos e posteriormente comparados utilizando-se curvas $\mathrm{NH}$, em que valores muito próximos a 1,0 refletem layouts com boa capacidade de preservação de classes.

Os estudos de caso apresentados ilustram que visualizações geradas com o modelo vetorial apresentaram os melhores resultados. Algumas métricas baseados em strings também obtiveram bons resultados nas comparações, em especial as medidas Qgram, Cosseno e Coeficiente de Overlap. A grande vantagem das métricas baseadas em strings é que não requerem da criação de um representação intermediária para o conjunto de textos, tais como o modelo vetorial. No entanto, os cálculos de distância tem um elevado custo computacional.

Foi descrito também uma possível modificação do VSM para representar uma coleção dinâmica de documentos textuais. O iVSM, proposto inicialmente por Pinho (2009), constrói um modelo vetorial que pode ser atualizado conforme os documentos são adicionados 
à coleção. Resultados na análise realizada, em relação à capacidade de segregação das classes definidas nos conjuntos de textos, ilustram que iVSM superou o VSM em quase todos os casos, sendo que em outros se colocou muito perto dele. Desde que o VSM não é incremental, ao passo em que muitos dos conjuntos de dados o são, o iVSM converte-se no principal candidato na geração de representações visuais, junto com as técnicas incrementais Multidimensional Scaling (MDS) como incBoard e incSpace (Pinho et al., 2010), de fluxos de texto e coleções de documentos variante no tempo, em que poucos novos cálculos são necessários.

Adicionalmente, dois módulos foram adicionados à ferramenta VisPipeline: o primeiro denominado String Metrics que computa o cálculo da dissimilaridade entre documentos utilizando medidas de comparação de strings; e um segundo que computa o iVSM para os documentos de uma coleção.

Finalmente, um novo conjunto de dados classificado foi gerado neste trabalho, o qual pode ser útil para outros trabalhos do VICG. O conjunto denominado News2011 está disponibilizado em http://infoserver.lcad.icmc.usp.br/infovis2/DataSets.

\subsection{Limitações e Trabalhos Futuros}

Em termos de limitações, todas as representações e abordagens aqui estudadas desconsideram qualquer tipo de análise semântica. Por exemplo, a utilização de lematização de palavras no pré-processamento dos dados impacta na análise semântica, embora de uma forma não muito previsível. Entretanto, esse tipo de processamento e cálculo de dissimilaridade é suficiente para muitas aplicações, além de ser um passo necessário quando o conteúdo dos textos é desconhecido. Representações baseadas na semântica e cálculos de distância nesse cenário são questões que precisam ser mais bem investigadas. Algumas aplicações são altamente dependentes de interpretação semântica e não se pode proporcionar um tratamento independente dela.

Precisa-se também de um estudo sistemático do impacto do modelo de linguagem para conjuntos de textos não homogêneos, sendo que, a principal limitação dos modelos de linguagem é o seu tamanho, que aumenta consideravelmente quando novos tópicos aparecem no conjunto de dados.

Um passo seguinte é avaliar o iVSM e as medidas de comparação de strings em uma configuração verdadeiramente incremental, aplicando-os na exibição de fluxos de texto como, utilizando por exemplo, incBoard ou outras técnicas incrementais. 


\section{Referências Bibliográficas}

Albrecht-Buehler, C.; Watson, B.; Shamma, D. A. Visualizing Live Text Streams Using Motion and Temporal Pooling. IEEE Computer Graphics and Applications, v. 25, n. 3, p. 52-59, 2005.

Disponível em http://ieeexplore.ieee.org/xpls/abs_all.jsp?arnumber= 1438258

Almeida, L. J.; Lopes, A. A. An Ultra-Fast Modularity-Based Graph Clustering Algorithm. In: Proceedings in 14th Portuguese Conference on Artificial Intelligence, 2009, p. 1-9.

Disponível em http://www.icmc.usp.br/〜alneu/papers2007_2009/ WNI2009Almeida_Lopes.pdf

Apté, C.; Damerau, F.; Weiss, S. M. Automated learning of decision rules for text categorization. ACM Transactions on Information Systems, v. 12, n. 3, p. 233-251, 1994a.

Disponível em http://doi.acm.org/10.1145/183422.183423

Apté, C.; Damerau, F.; Weiss, S. M. Towards language independent automated learning of text categorization models. In: Proceedings of the 17th annual international ACM SIGIR conference on Research and development in information retrieval, 1994b, p. $23-30$.

Disponível em http://dl .acm.org/citation. cfm?id=188490.188497

Baeza-Yates, R. A.; Ribeiro-Neto, B. Modern Information Retrieval. AddisonWesley Longman Publishing Co., Inc., 1999. 
Card, S. K.; Mackinlay, J. D.; Shneiderman, B. Readings in Information Visualization: Using Vision to Think. Morgan Kaufmann Publishers Inc., 1999.

Chen, C. Information Visualization: Beyond the Horizon. Springer-Verlag New York, Inc., 2006.

Cilibrasi, R.; Vitanyi, P. Clustering by compression. IEEE Transactions on Information Theory, v. 51, n. 4, p. 1523-1545, 2005.

Disponível em http://ieeexplore.ieee.org/xpls/abs_all.jsp?arnumber= 1412045\&tag=1

Cohen, W.; Ravikumar, P.; Fienberg, S. A Comparison of String Metrics for Matching Names and Records. In: Proceedings of the 2003 ACM SIGKDD Workshop on Data Cleaning, Record Linkage, and Object Consolidation, 2003, p. 13-18.

Disponível em http://www.cs.cmu.edu/ ^wcohen/postscript/kdd-2003-match-ws. pdf

Cuadros, A. M. Mapeamento de dados multidimensionais usando árvores filogenéticas: foco em mapeamento de textos. Dissertação de Mestrado, Instituto de Ciências Matemáticas e de Computação - Universidade de São Paulo, 2007.

Disponível em http://www.teses.usp.br/teses/disponiveis/55/55134/ tde-22012008-111242/pt-br.php

Cuadros, A. M.; Paulovich, F. V.; Minghim, R.; Telles, G. P. Point Placement by Phylogenetic Trees and its Application to Visual Analysis of Document Collections. In: Proceedings of the 2007 IEEE Symposium on Visual Analytics Science and Technology, 2007, p. 99-106.

Disponível em http://dx.doi.org/10.1109/VAST.2007.4389002

Cui, W.; Wu, Y.; Liu, S.; WeI, F.; Zhou, M.; Qu, H. Context-Preserving, Dynamic Word Cloud Visualization. IEEE Computer Graphics and Applications, v. 30, n. 6 , p. 42-53, 2010.

Disponível em http://ieeexplore.ieee.org/xpls/abs_all.jsp?arnumber= 5567084

Das, G.; Fleischer, R.; Gasieniec, L.; Gunopulos, D.; KärkkÄInen, J. Episode Matching. In: Proceedings of the 8th Annual Symposium on Combinatorial Pattern Matching, 1997, p. 12 - 27.

Disponível em http://dl .acm.org/citation. cfm?id=647816.738462 
Faloutsos, C.; Lin, K.-I. FastMap: A Fast Algorithm for Indexing, Data Mining and Visualization of Tradicional and Multidimedia Datasets. ACM SIGMOD Record, v. 24, n. 2, p. 163-174, 1995.

Disponível em http://doi.acm.org/10.1145/568271.223812

Fayyad, U.; Grinstein, G. G.; Wierse, A. Information visualization in data mining and knowledge discovery. Morgan Kaufmann Publishers Inc., 2002.

Fitch, W. M.; Margoliash, E. Construction of Phylogenetic Trees. Science, v. 155, n. 3760, p. 279-284, 1967.

Disponível em http://www.sciencemag.org/content/155/3760/279. citation

Fox, C. A stop list for general text. SIGIR Forum, v. 24, n. 1-2, p. 19-21, 1989. Disponível em http://doi.acm.org/10.1145/378881.378888

Gravano, L.; Ipeirotis, P. G.; Jagadish, H. V.; Koudas, N.; Muthukrishnan, S.; Pietarinen, L.; Srivastava, D. Using q-grams in a DBMS for Approximate String Processing. IEEE Data Engineering Bulletin, v. 24, n. 4, p. 28-34, 2001.

Disponível em http://sites . computer .org/debull/A01DEC-CD.pdf

Hassan-Montero, Y.; Herrero-Solana, V. Improving Tag-Clouds as Visual Information Retrieval Interfaces. In: International Conference on Multidisciplinary Information Sciences and Technologies, 2006, p. 25-28.

Disponível em http://users.cs.fiu.edu/〜yzhan004/rectangularPacking/ tagcloudsImproving.pdf

Havre, S.; Hetzler, E.; Whitney, P.; Nowell, L. ThemeRiver: Visualizing Thematic Changes in Large Document Collections. IEEE Transactions on Visualization and Computer Graphics, v. 8, n. 1, p. 9-20, 2002.

Disponível em http://dx.doi.org/10.1109/2945.981848

Hayes, P. J.; Andersen, P. M.; Nirenburg, I. B.; Schmandt, L. M. TCS: a shell for content-based text categorization. In: Proceedings of the sixth conference on Artificial intelligence applications, 1990, p. 320-326.

Disponível em http://dl.acm.org/citation.cfm?id=96751.96803

Hayes, P. J.; Weinstein, S. P. COnStRUE/TIS: A System for Content-Based Indexing of a Database of News Stories. In: Proceedings of the The Second Conference on Innovative Applications of Artificial Intelligence, 1991, p. 49-64.

Disponível em http://dl . acm.org/citation. cfm? id=645450.653070 
Hopper, E.; Turton, B. C. H. An Empirical Investigation of Meta-heuristic and Heuristic Algorithms for a 2D Packing Problem. European Journal of Operational Research, v. 128, n. 1, p. $34-57,2001$.

Disponível em http://www.sciencedirect.com/science/article/pii/ S0377221799003574

Huang, Y.; Madey, G. Web data integration using approximate string join. In: Proceedings of the 13th international World Wide Web conference on Alternate track papers $\&$ posters, 2004, p. 364-365.

Disponível em http://doi.acm.org/10.1145/1013367.1013477

Huh, S.; Fienberg, S. E. Discriminative Topic Modeling Based on Manifold Learning. ACM Transactions on Knowledge Discovery from Data, v. 5, n. 4, p. 20:1-20:25, 2012. Disponível em http://doi.acm.org/10.1145/2086737.2086740

Kaufman, L.; Rousseeuw, P. J. Finding Groups in Data: An Introduction to Cluster Analysis. Wiley-Interscience, 2005.

Kempken, S.; Luther, W.; Pilz, T. Comparison of distance measures for historical spelling variants. In: Artificial Intelligence in Theory and Practice, 2006, p. 295-304. Disponível em http://dx.doi.org/10.1007/978-0-387-34747-9_31

Korf, R. E. Optimal Rectangle Packing: New Results. In: Proceedings of the 14th International Conference on Automated Planning and Scheduling, 2004, p. 142-149. Disponível em http://www . aaai .org/Papers/ICAPS/2004/ICAPS04-019.pdf

Levenshtein, V. I. Binary codes with correction for deletions and insertions of the symbol 1. Problems of Information Transmission, v. 1, n. 1, p. 12-25, 1965.

Disponível em http://mi.mathnet.ru/eng/ppi69

Levenshtein, V. I. Binary codes capable of correcting deletions, insertions, and reversals. Soviet Physics Doklady, v. 10, n. 8, p. 707-710, 1966.

Li, M.; Chen, X.; Li, X.; Ma, B.; Vitanyi, P. The similarity metric. IEeE Transactions on Information Theory, v. 50, n. 12, p. 3250-3264, 2004.

Disponível em http://ieeexplore.ieee.org/xpls/abs_all.jsp?arnumber= 1362909

Lopes, A. A.; Minghim, R.; Melo, V.; Paulovich, F. V. Mapping Texts Through Dimensionality Reduction and Visualization Techniques for Interactive Exploration of 
Document Collections. In: Proceedings of SPIE-ISET Electronic Imaging - Workshop on Visualization and Data Analysis, 2006, p. 60600T-1-60600T12.

Disponível em http://dx.doi.org/10.1117/12.650899

Lopes, A. A.; Pinho, R. D. D.; Paulovich, F. V.; Minghim, R. Visual text mining using association rules. Computers and Graphics, v. 31, n. 3, p. 316-326, 2007. Disponível em http://dx.doi.org/10.1016/j.cag.2007.01.023

Luhn, H. P. The automatic creation of literature abstracts. IBM Journal of Research and Development, v. 2, n. 2, p. 159-165, 1958.

Disponível em http://dx.doi.org/10.1147/rd.22.0159

Mailund, T.; Brodal, G. S.; Fagerberg, R.; Pedersen, C. N. S.; Phillips, D. Recrafting the Neighbor-Joining Method. BMC Bioinformatics, v. 7, n. 29, 2006.

Disponível em http://www. biomedcentral . com/1471-2105/7/29

Minghim, R.; Paulovich, F. V.; Lopes, A. A. Content-based Text Mapping using Multi-dimensional Projections for Exploration of Document Collections. Visualization and Data Analysis, v. 6060, p. 259-270, 2006.

Disponível em https://www.icmc.usp.br/〜alneu/papers/minghim_etal2006.pdf

Motta, R.; Lopes, A. A.; Oliveira, M. C. F. Centrality Measures from Complex Networks in Active Learning. In: Proceedings of the 12th International Conference on Discovery Science, 2009, p. 184-196.

Disponível em http://dx.doi.org/10.1007/978-3-642-04747-3_16

Needleman, S. B.; Wunsch, C. D. A general method applicable to the search for similarities in the amino acid sequence of two proteins. Journal of Molecular Biology, v. 48, n. 3, p. 443-453, 1970.

Disponível em http://www.sciencedirect.com/science/article/pii/ 0022283670900574

Oliveira, M. C. F.; Levkowitz, H. From visual data exploration to visual data mining: a survey. IEEE Transactions on Visualization and Computer Graphics, v. 9, n. 3, p. 378-394, 2003.

Disponível em http://ieeexplore.ieee.org/xpls/abs_all.jsp?arnumber= 1207445 
Paiva, J.; Florian, L.; Pedrini, H.; Telles, G. P.; Minghim, R. Improved Similarity Trees and their Application to Visual Data Classification. IEEE Transactions on Visualization and Computer Graphics, v. 17, n. 12, p. 2459-2468, 2011.

Disponível em http://ieeexplore.ieee.org/xpls/abs_all.jsp?arnumber= 6065013

Paulovich, F. V. Mapeamento de dados multi-dimensionais - integrando mineração e visualização. Tese de Doutoramento, Instituto de Ciências Matemáticas e de Computação - Universidade de São Paulo, 2008.

Disponível em http://www.teses.usp.br/teses/disponiveis/55/55134/ tde-04032009-145018/pt-br.php

Paulovich, F. V.; Minghim, R. Text Map Explorer: a Tool to Create and Explore Document Maps. In: Proceedings of the Conference on Information Visualization, 2006, p. 245-251.

Disponível em http://dx.doi.org/10.1109/IV.2006.104

Paulovich, F. V.; Nonato, L. G.; Minghim, R. Visual Mapping of Text Collections through a Fast High Precision Projection Technique. In: Proceedings of the Conference on Information Visualization, 2006, p. 282-290.

Disponível em http://dx.doi.org/10.1109/IV.2006.122

Paulovich, F. V.; Nonato, L. G.; Rosane, M.; Levkowitz, H. Least Square Projection: A Fast High-Precision Multidimensional Projection Technique and Its Application to Document Mapping. IEEE Transactions on Visualization and Computer Graphics, v. 14, n. 3, p. 564-575, 2008.

Disponível em http://ieeexplore.ieee.org/xpls/abs_all.jsp?arnumber= 4378370

Paulovich, F. V.; Oliveira, M. C. F.; Minghim, R. The Projection Explorer: A flexible tool for projection-based multidimensional visualization. In: Proceedings of the XX Brazilian Symposium on Computer Graphics and Image Processing, 2007, p. $27-36$.

Disponível em http://dx.doi.org/10.1109/SIBGRAPI.2007.39

Paulovich, F. V.; Toledo, F. M. B.; Telles, G. P.; Minghim, R.; Nonato, L. G. Semantic Wordification of Document Collections. Computer Graphics Forum, v. 31, n. 3pt3, p. 1145-1153, 2012.

Disponível em http://dx.doi.org/10.1111/j.1467-8659.2012.03107.x 
Pinho, R. D. D. Espaço incremental para a mineração visual de conjuntos dinâmicos de documentos. Tese de Doutoramento, Instituto de Ciências Matemáticas e de Computação - Universidade de São Paulo, 2009.

Disponível em http://www.teses.usp.br/teses/disponiveis/55/55134/ tde-14092009-123807/pt-br.php

Pinho, R. D. D.; Oliveira, M. C. F.; Lopes, A. A. Incremental Board: A Gridbased Space for Visualizing Dynamic Data Sets. In: Proceedings of the 2009 ACM symposium on Applied Computing, 2009, p. 1757-1764.

Disponível em http://doi.acm.org/10.1145/1529282.1529679

Pinho, R. D. D.; Oliveira, M. C. F.; Lopes, A. A. An incremental space to visualize dynamic data sets. Multimedia Tools and Applications, v. 50, n. 3, p. 533$562,2010$.

Disponível em http://dx.doi.org/10.1007/s11042-010-0483-5

Porter, M. F. Readings in information retrieval. cáp. An algorithm for suffix stripping, Morgan Kaufmann Publishers Inc., p. 313-316, 1997.

Disponível em http://dl .acm.org/citation. cfm?id=275537.275705

SAitou, N.; NeI, M. The neighbor-joining method: a new method for reconstructing phylogenetic trees. Molecular Biology and Evolution, v. 4, n. 4, p. 406-425, 1987.

Disponível em http://mbe.oxfordjournals .org/content/4/4/406.full .pdf+html

Salton, G.; Wong, A.; YAng, C. S. A vector space model for automatic indexing. Communications of the ACM, v. 18, n. 11, p. 613-620, 1975.

Disponível em http://doi.acm.org/10.1145/361219.361220

SANkofF, D.; Kruskal, J. Time warps, string edits, and macromolecules: the theory and practice of sequence comparison. Addison-Wesley Press, 1983.

Schütze, H. Automatic word sense discrimination. Computational Linguistics, v. 24, n. 1, p. 97-123, 1998.

Disponível em http://dl .acm.org/citation. cfm?id=972719.972724

Seifert, C.; Kump, B.; Kienreich, W.; Granitzer, G.; Granitzer, M. On the Beauty and Usability of Tag Clouds. In: Proceedings in 12th International Conference Information Visualisation, 2008, p. 17-25.

Disponível em http://dx.doi.org/10.1109/IV.2008.89 
Selvaraj, S. K.; Bhar, B.; Sellamanickam, S.; Shevade, S. Semi-supervised SVMs for classification with unknown class proportions and a small labeled dataset. In: Proceedings of the 20th ACM international conference on Information and knowledge management, 2011, p. 653-662.

Disponível em http://doi.acm.org/10.1145/2063576.2063674

Setubal, C.; Meidanis, J. Introduction to Computational Molecular Biology. PWS Publishing, 1997.

Shewchuk, J. R. An Introduction to the Conjugate Gradient Method Without the Agonizing Pain. Relatório Técnico, Carnegie Mellon University, 1994.

Disponível em http://www.cs.cmu.edu/ quake-papers/ painless-conjugate-gradient.pdf

Singleton, P. Dictionary of DNA and Genome Technology. John Wiley \& Sons Ltd, 2010.

Smith, T.; Waterman, M. Identification of Common Molecular Subsequences. Journal of Molecular Biology, v. 147, n. 1, p. 195-197, 1981.

Disponível em http://dx.doi.org/10.1016/0022-2836(81)90087-5

Srivastava, A.; Sahami, M. Text mining: classification, clustering, and applications. Chapman \& Hall/CRC Data Mining and Knowledge Discovery Series, 2009.

Steinbach, M.; Karypis, G.; Kumar, V. A Comparison of Document Clustering Techniques. In: KDD Workshop on Text Mining, 2000, p. 525-526.

Disponível em http://glaros.dtc.umn.edu/gkhome/fetch/papers/ docclusterKDDTMW00.pdf

Subramanya, A.; Bilmes, J. Soft-supervised learning for text classification. In: Proceedings of the Conference on Empirical Methods in Natural Language Processing, 2008, p. 1090-1099.

Disponível em http://dl .acm.org/citation.cfm?id=1613715.1613857

Tan, P.-N.; Steinbach, M.; Kumar, V. Introduction to Data Mining, (First Edition). Addison-Wesley, 2005.

Tejada, E.; Minghim, R.; Nonato, L. G. On improved projection techniques to support visual exploration of multidimensional data sets. Information Visualization, 
v. 2, n. 4, p. 218-231, 2003.

Disponível em http://dx.doi.org/10.1057/palgrave.ivs.9500054

Telles, G. P.; Minghim, R.; Paulovich, F. V. Visual Analytics: Normalized compression distance for visual analysis of document collections. Computers and Graphics, v. 31, n. 3, p. 327-337, 2007.

Disponível em http://dx.doi.org/10.1016/j.cag.2007.01.024

Thomas, J. J.; Cook, K. A. A visual analytics agenda. IEEE Computer Graphics and Applications, v. 26, n. 1, p. 10-13, 2006.

Disponível em http://ieeexplore.ieee.org/xpls/abs_all.jsp?arnumber= 1573625

Wäscher, G.; HauBner, H.; Schumann, H. An improved typology of cutting and packing problems. European Journal of Operational Research, v. 183, n. 3, p. 1109-1130, 2007.

Disponível em http://www.sciencedirect.com/science/article/pii/ S037722170600292X

Wei, F.; Liu, S.; Song, Y.; Pan, S.; Zhou, M. X.; Qian, W.; Shi, L.; Tan, L.; ZHANG, Q. TIARA: a visual exploratory text analytic system. In: Proceedings of the 16th ACM SIGKDD international conference on Knowledge discovery and data mining, 2010, p. 153-162.

Disponível em http://doi.acm.org/10.1145/1835804.1835827

Wilbur, W. J.; Sirotkin, K. The automatic identification of stop words. Journal of Information Science, v. 18, n. 1, p. 45-55, 1992.

Disponível em http://dx.doi.org/10.1177/016555159201800106

YAnG, T.; LeE, D. Towards noise-resilient document modeling. In: Proceedings of the 20th ACM international conference on Information and knowledge management, 2011, p. $2345-2348$.

Disponível em http://doi.acm.org/10.1145/2063576.2063962

ZIPF, G. K. Human Behavior and the Principle of Least Effort: An Introduction to Human Ecology. Addison-Wesley Press, 1949. 